\title{
Homotopy theory of nonsymmetric operads
}

\author{
FERnANDo Muro
}

\begin{abstract}
We endow categories of nonsymmetric operads with natural model structures. We work with no restriction on our operads and only assume the usual hypotheses for model categories with a symmetric monoidal structure. We also study categories of algebras over these operads in enriched nonsymmetric monoidal model categories.
\end{abstract}

18D50, 55U35; 18D10, 18D35, 18D20

\section{Introduction}

Operads are well-known devices encoding the laws of algebras defined by multilinear operations and relations, eg there are operads Ass, Com and Lie whose algebras are associative, commutative and Lie algebras, respectively. Morphisms of operads codify relations between different kinds of algebras, eg there are morphisms Lie $\rightarrow$ Ass $\rightarrow$ Com telling us that any commutative algebra is an associative algebra, and that commutators in an associative algebra yield a Lie algebra.

There are two kinds of operads: symmetric and nonsymmetric operads. Symmetric operads are needed whenever it is necessary to permute variables in order to describe the laws of the corresponding algebras, eg Com and Lie. Nonsymmetric operads are specially useful to deal with algebras in nonsymmetric monoidal categories, eg given a commutative ring $k$ and a set $S$ which is not a singleton, the category of $k$-modules with object set $S$, which are collections of $k$-modules indexed by $S \times S$, $M=\{M(x, y)\}_{x, y \in S}$, has a nonsymmetric tensor product,

$$
\left(M \otimes_{S} N\right)(x, y)=\bigoplus_{z \in S} M(z, y) \otimes_{k} N(x, z),
$$

whose associative algebras, ie algebras over the operad Ass, are $k$-linear categories with object set $S$.

Any object $M$ in a symmetric monoidal category $\mathscr{V}$, such as the category of $k-$ modules, has an endomorphism symmetric operad $\operatorname{End}_{\mathcal{V}}(M)$ in $\mathscr{V}$ such that, if $\mathcal{O}$ is another symmetric operad in $\mathscr{V}$, the set of $\mathcal{O}$-algebra structures on $M$ is the set of symmetric operad morphisms $\mathcal{O} \rightarrow \operatorname{End}_{\mathcal{V}}(M)$. If $M$ belongs to a nonsymmetric 
monoidal category $\mathscr{C}$ enriched over $\mathscr{V}$, such as the category of $k$-modules with object set $S$, then there is a nonsymmetric operad $\operatorname{End}_{\mathscr{C}}(M)$ in $\mathscr{V}$ such that the set of algebra structures on $M$ over a nonsymmetric operad $\mathcal{O}$ in $\mathscr{V}$ is the set of nonsymmetric operad morphisms $\mathcal{O} \rightarrow \operatorname{End}_{\mathscr{C}}(M)$.

When the underlying symmetric monoidal category $\mathscr{V}$ carries homotopical information, eg if we replace $k$-modules with differential graded $k$-modules, one is often more interested in a space of $\mathcal{O}$-algebra structures on $M$ rather than a plain set. Such a space can be constructed by using the powerful machinery developed by Dwyer and Kan $[9 ; 7 ; 8]$ provided we can place the operads $\mathcal{O}$ and $\operatorname{End}_{\mathscr{C}}(M)$ in an appropriate model category of operads.

Model categories of operads were first considered by Hinich in the differential graded context [13; 12], and by Berger and Moerdijk in a more general setting [4]. They dealt with symmetric operads and showed that restrictive hypotheses are necessary to endow the category of all operads with an appropriate model category structure, eg when $k$ is a $\mathbb{Q}$-algebra or when the symmetric monoidal structure in $\mathscr{V}$ is cartesian closed and there is a symmetric monoidal fibrant replacement functor.

Motivated by our interest in spaces of differential graded category structures, we consider the nonsymmetric case, which surprisingly enough does not need any restrictive hypotheses, just usual hypotheses for model categories with a monoidal structure; see Schwede and Shipley [20].

Theorem 1.1 Let $\mathscr{V}$ be a cofibrantly generated closed symmetric monoidal model category. Assume that $\mathscr{V}$ satisfies the monoid axiom. Moreover, suppose that there are sets of generating cofibrations and generating trivial cofibrations in $\mathscr{V}$ with presentable sources. Then the category $\mathrm{Op}(\mathscr{V})$ of nonsymmetric operads in $\mathscr{V}$ is a cofibrantly generated model category such that a morphism $f: \mathcal{O} \rightarrow \mathcal{P}$ in $\mathrm{Op}(\mathscr{V})$ is a weak equivalence (resp. fibration) if and only if $f(n): \mathcal{O}(n) \rightarrow \mathcal{P}(n)$ is a weak equivalence (resp. fibration) in $\mathscr{V}$ for all $n \geq 0$. Moreover, if $\mathscr{V}$ is right proper then so is $\operatorname{Op}(\mathscr{V})$. Furthermore, if $\mathscr{V}$ is combinatorial then $\mathrm{Op}(\mathscr{V})$ is also combinatorial.

This theorem can be applied to all examples in [20] (see also the references therein):

(1) Complexes of modules over a commutative ring $k$ with the usual tensor product of complexes.

(2) Simplicial $k$-modules with the levelwise tensor product $\otimes_{k}$.

(3) Modules over a finite-dimensional Hopf algebra $R$ over a field $k$ with the tensor product over $k$, eg $R=k G$ the group-ring of a finite group $G$.

(4) Symmetric spectra with their smash product, and more generally modules over a commutative ring spectrum. 
(5) $\Gamma$-spaces with Lydakis' smash product.

(6) Simplicial functors with their smash product.

(7) $S$-modules with their smash product.

In particular, Theorem 1.1 will also be useful to study spaces of spectral category structures.

Recall from Adámek and Rosický [1, Definition 1.13 (2)] that an object $X$ of $\mathscr{V}$ is presentable if there exists a cardinal $\lambda$ such that the representable functor $\mathscr{V}(X,-)$ commutes with $\lambda$-filtered colimits in $\mathscr{V}$. Presentable objects are also called small or compact in some references. All objects are presentable in many categories of interest, eg in all combinatorial model categories. Actually, up to set theoretical principles any cofibrantly generated model category is Quillen equivalent to a combinatorial model category; see Raptis [19].

Categories of algebras over symmetric operads do not always have a model structure with fibrations and weak equivalences defined in the underlying category. Sufficient conditions can be found in Berger and Moerdijk [4]. In the framework of nonsymmetric operads they do. When both algebras and operads live in the same ambient symmetric monoidal model category $\mathscr{V}$, satisfying the monoid axiom, this has been recently proved by JE Harper [11, Theorem 1.2]. We here extend this result to algebras in a monoidal model category $\mathscr{b}$ satisfying the monoid axiom and appropriately enriched over $\mathscr{V}$. This is necessary, for instance, to construct model categories of enriched categories, of enriched $A_{\infty}$-categories, or of any other categorified algebraic structure; see Section 10.

Theorem 1.2 Let $\mathscr{V}$ and $\mathscr{C}$ be cofibrantly generated biclosed monoidal model categories. Suppose $\mathscr{V}$ is symmetric and $\mathscr{C}$ has a $\mathscr{V}$-algebra structure given by a strong braided monoidal functor $z: \mathscr{V} \rightarrow Z(\mathscr{C})$ to the center of $\mathscr{C}$ such that the composite functor

$$
\mathscr{V} \stackrel{z}{\longrightarrow} Z(\mathscr{C}) \stackrel{\text { forget }}{\longrightarrow} \mathscr{C}
$$

is a left Quillen functor. Moreover, assume that $\mathscr{V}$ and $\mathscr{C}$ satisfy the monoid axiom (see Definition 6.1 and Definition 9.1). Furthermore, suppose that $\mathscr{C}$ has sets of generating cofibrations and generating trivial cofibrations with presentable source. Let $\mathcal{O}$ be a nonsymmetric operad in $\mathscr{V}$. The category $\operatorname{Alg}_{\mathscr{C}}(\mathcal{O})$ of $\mathcal{O}$-algebras in $\mathscr{C}$ is a cofibrantly generated model category such that an $\mathcal{O}$-algebra morphism $g: A \rightarrow B$ is a weak equivalence (resp. fibration) if and only if $g$ is a weak equivalence (resp. fibration) in $\mathscr{C}$. Moreover, if $\mathscr{C}$ is right proper then so is $\operatorname{Alg}_{\mathscr{C}}(\mathcal{O})$. Furthermore, if $\mathscr{C}$ is combinatorial then $\operatorname{Alg}_{\mathscr{C}}(\mathcal{O})$ is also combinatorial. 
The notion of monoidal model category in [20, Definition 3.1] makes sense with no modification in the nonsymmetric context; see Definition 4.2.

Any operad morphism $\phi: \mathcal{O} \rightarrow \mathcal{P}$ induces a change of operad functor

$$
\phi^{*}: \operatorname{Alg}_{\mathscr{C}}(\mathcal{P}) \longrightarrow \operatorname{Alg}_{\mathscr{C}}(\mathcal{O})
$$

by restricting the action of $\mathcal{P}$ to $\mathcal{O}$ along $\phi$. This functor is the identity on underlying objects in $\mathscr{C}$, hence it preserves fibrations and weak equivalences. Moreover, the functor $\phi^{*}$ has a left adjoint $\phi_{*}$, therefore we have a Quillen adjunction

$$
\operatorname{Alg}_{\mathscr{C}}(\mathcal{O}) \underset{\phi^{*}}{\stackrel{\phi_{*}}{\rightleftarrows}} \operatorname{Alg}_{\mathscr{C}}(\mathcal{P})
$$

The following result establishes conditions so that this is a Quillen equivalence if $\phi$ is a weak equivalence of operads. These conditions are the nonsymmetric analogues of those considered in [4] for symmetric operads.

Theorem 1.3 In the conditions of the previous theorem, assume further that $\mathscr{b}$ is left proper. Let $\phi: \mathcal{O} \rightarrow \mathcal{P}$ be a weak equivalence between operads in $\mathscr{V}$ such that for all $n \geq 0$ the objects $\mathcal{O}(n)$ and $\mathcal{P}(n)$ are cofibrant in $\mathscr{V}$. Then Equation (1) is a Quillen equivalence, in particular the derived adjoint pair is an equivalence between the homotopy categories of algebras:

$$
\operatorname{Ho} \operatorname{Alg}_{\mathscr{C}}(\mathcal{O}) \underset{\phi^{*}}{\stackrel{\mathbb{L} \phi_{*}}{\rightleftarrows}} H_{o} \operatorname{Alg}_{\mathscr{C}}(\mathcal{P}) \text {. }
$$

This result will be useful to show that in many examples the homotopy theory of enriched categories coincides with the homotopy theory of $A_{\infty}$-categories, eg when the underlying symmetric monoidal category $\mathscr{V}$ is any of the categories in the examples (1)-(6) listed above; see Section 10.

When $\mathscr{C}$ is a simplicial model category and the simplicial structure is compatible with $z: \mathscr{V} \rightarrow Z(\mathscr{C})$ in a suitable way, the derived equivalence of homotopy categories in Theorem 1.3 was obtained by Batanin in [2, Section 2] using totally different methods closer to categorical algebra than to homotopy theory.

The paper is structured as follows. Sections 2, 5 and 6 deal with operads and Sections 7, 8 and 9 deal with algebras in a rather parallel way: we recall the basics on these algebraic structures, we give very detailed constructions of some pushouts which are the main ingredients for the proofs of our main theorems, and then we proceed with the proofs. The other sections are auxiliary. 
Acknowledgements The author wishes to thank Michael Batanin, Clemens Berger, Benoit Fresse, Javier J Gutiérrez, Ieke Moerdijk, Andy Tonks and Bruno Vallette for conversations related to the contents of this paper, in particular for providing very interesting references. FM was partially supported by the Spanish Ministry of Education and Science under the MEC-FEDER grants MTM2007-63277 and MTM2010-15831, by the Government of Catalonia under the grant SGR-119-2009 and by the Andalusian Ministry of Economy, Innovation and Science under the grant FQM-5713.

Notation Throughout this paper $\mathscr{V}$ and $\mathscr{C}$ will denote complete and cocomplete biclosed monoidal categories (see Kelly [17, 1.5]) with tensor product $X \otimes Y$ and unit objects $\mathbb{I}_{\mathscr{V}}$ and $\mathbb{I}_{\mathscr{b}}$, respectively. We drop the subscript when it is clear from the context. The category $\mathscr{V}$ will be symmetric and internal morphism objects in $\mathscr{V}$ will be denoted by $\operatorname{Hom}(X, Y)$. We will add homotopical hypotheses when needed.

\section{Operads}

In this section we recall the well-known notion of nonsymmetric operad.

Definition 2.1 The category $\mathscr{V} \mathbb{N}$ of sequences of objects $V=\{V(n)\}_{n \geq 0}$ in $\mathscr{V}$ is the product of countably many copies of $\mathscr{V}$. It has a right-closed nonsymmetric monoidal structure given by the composition product $U \circ V$ (compare [2, Definition 1.2])

$$
(U \circ V)(m)=\coprod_{n \geq 0} \coprod_{\sum_{i=1}^{n} p_{i}=m} U(n) \otimes V\left(p_{1}\right) \otimes \cdots \otimes V\left(p_{n}\right) .
$$

The unit object is $\mathbb{I}_{\circ}$ :

$$
\mathbb{I}_{\circ}(n)= \begin{cases}\mathbb{I} & \text { the unit of } \otimes \text { in } \mathscr{V}, \text { if } n=1, \\ 0 & \text { the initial object of } \mathscr{V}, \text { if } n \neq 1\end{cases}
$$

Remark 2.2 The fact that $\circ$ is nonsymmetric is obvious from the very definition. One can easily check by writing down explicitly the formulas of $(U \circ V) \circ W$ and $U \circ(V \circ W)$ how the symmetry constraint of $\otimes$ is used to define the associativity constraint of $\circ$. The right adjoint of $-\circ V$ is the functor $\operatorname{Hom}_{\circ}(V,-)$ defined by

$$
\operatorname{Hom}_{\circ}(V, W)(n)=\prod_{p_{1}, \ldots, p_{n} \geq 0} \operatorname{Hom}\left(V\left(p_{1}\right) \otimes \cdots \otimes V\left(p_{n}\right), W\left(p_{1}+\cdots+p_{n}\right)\right),
$$

in particular $-\circ V$ preserves all colimits. On the contrary, the functor $U \circ-$ does not preserve all colimits, but it does preserve filtered colimits. 
Remark 2.3 If $\mathscr{V}$ is a model category then the product category $\mathscr{V} \mathbb{N}$ is also a model category with fibrations, cofibrations and weak equivalences defined coordinatewise [15, Example 1.1.6]. Moreover, if $\mathscr{V}$ is cofibrantly generated (resp. combinatorial) then $\mathscr{V}^{\mathbb{N}}$ is also cofibrantly generated (resp. combinatorial).

Indeed, let $I$ be a set of generating cofibrations and $J$ a set of generating trivial cofibrations in $\mathscr{V}$. For any $n \geq 0$, let $s_{n}: \mathscr{V} \rightarrow \mathscr{V} \mathbb{N}$ be the left adjoint of the projection onto the $n$-th factor, which is defined by

$$
\left(s_{n}(V)\right)(m)= \begin{cases}V & \text { if } m=n, \\ 0 & \text { the initial object, if } m \neq n\end{cases}
$$

Given a set $S$ of morphisms in $\mathscr{V}$ we consider the following set of morphisms in $\mathscr{V} \mathbb{N}$ :

$$
S_{\mathbb{N}}=\bigcup_{n \geq 0} s_{n}(S)
$$

The sets $I_{\mathbb{N}}$ and $J_{\mathbb{N}}$ are sets of generating cofibrations and generating trivial cofibrations in $\mathscr{V}^{\mathbb{N}}$, respectively.

Definition 2.4 A nonsymmetric operad $\mathcal{O}$ in $\mathscr{V}$ is a monoid in the monoidal category of sequences $\mathscr{V}^{\mathbb{N}}$ with the composition product $\circ$.

Remark 2.5 The previous condensed definition of an operad $\mathcal{O}$ can be unraveled by noticing that the multiplication $\mu: \mathcal{O} \circ \mathcal{O} \rightarrow \mathcal{O}$ consists of a series of multiplication morphisms, $1 \leq i \leq n, p_{i} \geq 0$,

$$
\mu_{n ; p_{1}, \ldots, p_{n}}: \mathcal{O}(n) \otimes \mathcal{O}\left(p_{1}\right) \otimes \cdots \otimes \mathcal{O}\left(p_{n}\right) \longrightarrow \mathcal{O}\left(p_{1}+\cdots+p_{n}\right) .
$$

The associativity condition amounts to saying that the following diagram is always commutative:

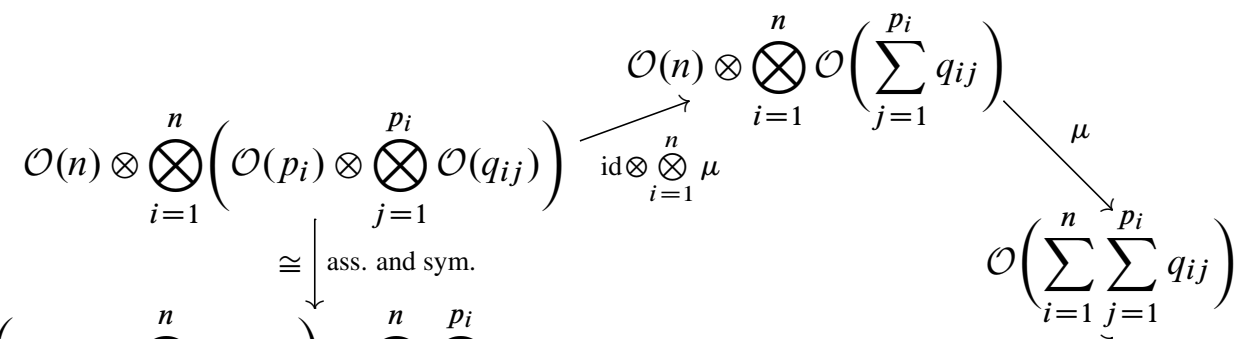

$$
\begin{aligned}
& \left(\mathcal{O}(n) \otimes \bigotimes_{i=1}^{n} \mathcal{O}\left(p_{i}\right)\right)^{\nu} \otimes \bigotimes_{i=1}^{n} \bigotimes_{j=1}^{p_{i}} \mathcal{O}\left(q_{i j}\right) \underbrace{\mu \otimes \mathrm{id}}_{n} \\
& \mathcal{O}\left(\sum_{i=1}^{n} p_{i}\right) \otimes \bigotimes_{i=1}^{n} \bigotimes_{j=1}^{p_{i}} \mathcal{O}\left(q_{i j}\right)
\end{aligned}
$$


Here the order of tensor factors in $\bigotimes_{i=1}^{n} \bigotimes_{j=1}^{p_{i}} \mathcal{O}\left(q_{i j}\right)$ is determined by the lexicographic order of the pair $(i, j)$. Moreover, the unit is just a morphism $u: \mathbb{I} \rightarrow \mathcal{O}(1)$ such that the following morphisms are (compositions of) unit constraints in $\mathscr{V}$ :

$$
\begin{gathered}
\mathbb{I} \otimes \mathcal{O}(n) \stackrel{u \otimes \mathrm{id}}{\longrightarrow} \mathcal{O}(1) \otimes \mathcal{O}(n) \stackrel{\mu_{1 ; n}}{\longrightarrow} \mathcal{O}(n), \\
\mathcal{O}(n) \otimes \mathbb{I}^{\otimes n} \stackrel{\mathrm{id} \otimes u^{\otimes n}}{\longrightarrow} \mathcal{O}(n) \otimes \mathcal{O}(1)^{\otimes n} \stackrel{\mu_{n ; 1, \ldots, 1}}{\longrightarrow} \mathcal{O}(n) .
\end{gathered}
$$

Remark 2.6 The multiplication morphisms in the previous remark are determined by the following morphisms, $1 \leq i \leq m, n \geq 0$,

$$
\circ_{i}: \mathcal{O}(m) \otimes \mathcal{O}(n) \longrightarrow \mathcal{O}(m+n-1),
$$

defined as

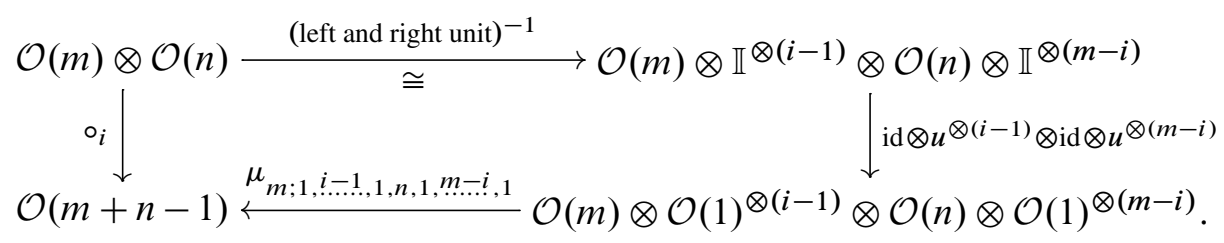

An operad can actually be defined as a collection of morphisms $\circ_{i}$ as above together with a unit morphism $u: \mathbb{I} \rightarrow \mathcal{O}$ (1) such that, for $1 \leq i \leq m$, the following diagrams commute:

(1) If $1 \leq j<i$ :

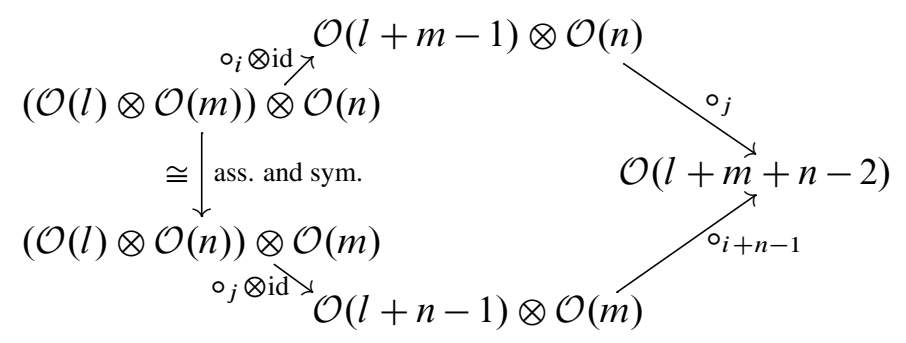

(2) If $i \leq j<m+i$ :

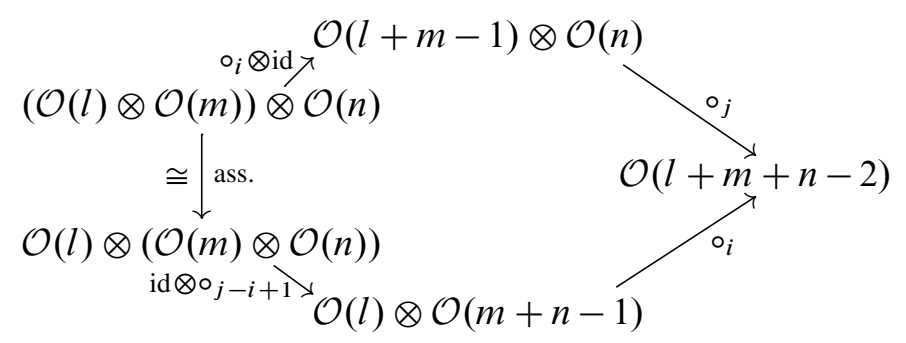


These relations are illustrated by the trees in Figure 10 below. Moreover, for all $1 \leq i \leq n$ the following composite morphisms must be unit constraints in $\mathscr{V}$ :

(3) $\quad \mathbb{I} \otimes \mathcal{O}(n) \stackrel{u \otimes \mathrm{id}}{\longrightarrow} \mathcal{O}(1) \otimes \mathcal{O}(n) \stackrel{\circ_{1}}{\longrightarrow} \mathcal{O}(n)$,

(4) $\mathcal{O}(n) \otimes \mathbb{I} \stackrel{\mathrm{id} \otimes u}{\longrightarrow} \mathcal{O}(n) \otimes \mathcal{O}(1) \stackrel{\circ_{i}}{\longrightarrow} \mathcal{O}(n)$.

\section{Trees}

The combinatorics of operads is that of trees with additional structure. In this section we recall some facts about trees that we need in order to prove our main theorems. We also give a different characterization of operads in terms of trees.

Definition 3.1 A planted tree is a contractible finite 1-dimensional simplicial complex $T$ with set of vertices $V(T)$, a nonempty set of edges $E(T)$, and a distinguished vertex $r(T) \in V(T)$ of degree 1, called root. Recall that the degree of $v \in V(T)$ is the number of edges containing $v$. Nevertheless, we will mostly use the number

$$
\tilde{v}=(\text { degree of } v)-1 .
$$

The level of a vertex $v \in V(T)$ is the distance to the root, level $(v)=d(v, r(T))$, with respect to the usual metric $d$ such that the distance between two adjacent vertices $\{v, w\} \in E(T)$ is $d(v, w)=1$. The height $\operatorname{ht}(T)$ of a planted tree $T$ is

$$
\operatorname{ht}(T)=\max _{v \in V(T)} \operatorname{level}(v) \text {. }
$$

Definition 3.2 A planted planar tree is a planted tree $T$ together with a total order $\leq$ in $V(T)$, called planar order, such that:

- If $\operatorname{level}(v)<\operatorname{level}(w)$ then $v<w$.

- If $\left\{v_{1}, v_{2}\right\},\left\{w_{1}, w_{2}\right\} \in E(T)$ are edges with

$$
\operatorname{level}\left(v_{1}\right)=\operatorname{level}\left(w_{1}\right)=\operatorname{level}\left(v_{2}\right)-1=\operatorname{level}\left(w_{2}\right)-1,
$$

and $v_{1}<w_{1}$, then $v_{2}<w_{2}$.

Given $e=\{v, w\} \in E(T)$ with $v<w$ we say that $e$ is an incoming edge of $v$ and the outgoing edge of $w$ (there is only one if $w \neq r(T)$ and none otherwise).

There is another useful order in $V(T)$ that we call the path order $\preceq$. Given $v \in V(T)$, consider the shortest path from $r(T)$ to $v$ and let $r(T)=v_{0}, \ldots, v_{n}=v$ be the vertices within this path in order of appearance. We associate with $v$ the word $v_{0} \cdots v_{n}$ in $V(T)$. The path order in $V(T)$ is the order induced by the lexicographic order of words in $V(T)$ with respect to $\leq$. 


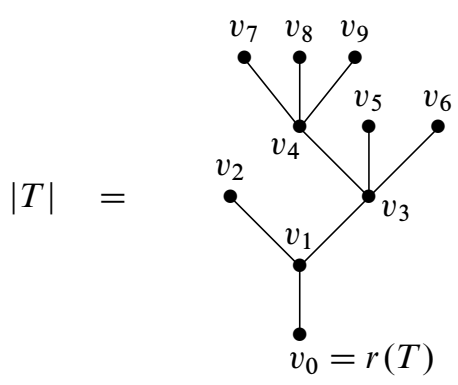

Figure 1: The geometric realization of a planted planar tree $T$ with vertices ordered by the subscript

Remark 3.3 Notice that the path order $\preceq$ restricted to level sets

$$
\{v \in V(T) ; \operatorname{level}(v)=n\}, \quad n \geq 0,
$$

coincides always with the planar order $\leq$.

The words associated to the vertices of the planted planar tree in Figure 1 are given in the following tables:

\begin{tabular}{c|c} 
vertex & word \\
\hline$v_{0}$ & $v_{0}$ \\
$v_{1}$ & $v_{0} v_{1}$ \\
$v_{2}$ & $v_{0} v_{1} v_{2}$ \\
$v_{3}$ & $v_{0} v_{1} v_{3}$ \\
$v_{4}$ & $v_{0} v_{1} v_{3} v_{4}$
\end{tabular}

\begin{tabular}{c|c} 
vertex & word \\
\hline$v_{5}$ & $v_{0} v_{1} v_{3} v_{5}$ \\
$v_{6}$ & $v_{0} v_{1} v_{3} v_{6}$ \\
$v_{7}$ & $v_{0} v_{1} v_{3} v_{4} v_{7}$ \\
$v_{8}$ & $v_{0} v_{1} v_{3} v_{4} v_{8}$ \\
$v_{9}$ & $v_{0} v_{1} v_{3} v_{4} v_{9}$
\end{tabular}

Hence the path order in $V(T)$ is $v_{0} \prec v_{1} \prec v_{2} \prec v_{3} \prec v_{4} \prec v_{7} \prec v_{8} \prec v_{9} \prec v_{5} \prec v_{6}$.

Definition 3.4 A planted planar tree with leaves is a planted planar tree $T$ together with a fixed set of degree 1 vertices $L(T)$, called leaves, different from the root, $r(T) \notin L(T)$. An inner vertex is a vertex which is neither a leaf nor the root. The set of inner vertices will be denoted by $I(T)$ and

$$
V(T)=\{r(T)\} \sqcup I(T) \sqcup L(T) .
$$

We denote by $\|T\|$ the open subspace of the geometric realization of $T$ obtained by removing the root and the leaves (see Figure 2):

$$
\|T\|=|T| \backslash(\{r(T)\} \sqcup L(T)) .
$$

Abusing of terminology, we say that an edge is the root or a leaf if it contains the root or a leaf vertex, respectively. The rest of edges are called inner edges. 


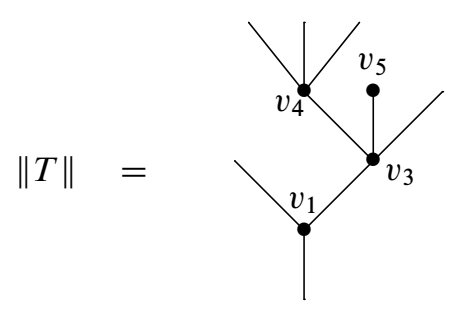

Figure 2: The space $\|T\|$ for the planted planar tree in Figure 1 with set of leaves $L(T)=\left\{v_{2}, v_{6}, v_{7}, v_{8}, v_{9}\right\}$

Given $n \geq 0$, the corolla with $n$ leaves is a planted planar tree $C_{n}$ with $n+2$ vertices and $n$ leaves; see Figure 3.

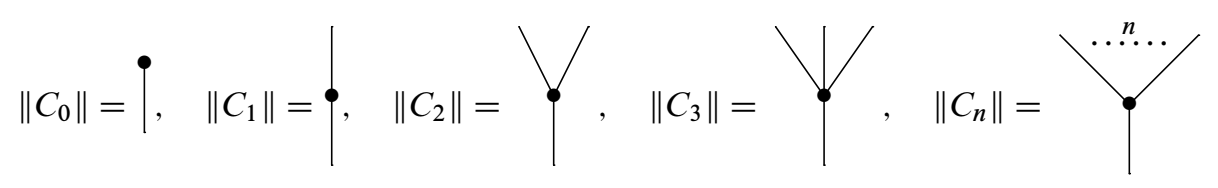

Figure 3: A class of planted planar trees with leaves: the corollas $C_{n}, n \geq 0$

A morphism of planted planar trees with leaves is a simplicial map $f: T \rightarrow T^{\prime}$ such that:

- If $v \preceq w \in V(T)$ then $f(v) \preceq f(w) \in V\left(T^{\prime}\right)$.

- $f^{-1}\left(\left\{r\left(T^{\prime}\right)\right\}\right)=\{r(T)\}$.

- $\quad \operatorname{card} L(T)=\operatorname{card} L\left(T^{\prime}\right)$ and $f^{-1}\left(L\left(T^{\prime}\right)\right)=L(T)$.

We denote by PPTL the category of planted planar trees with leaves. Notice that this category has no nontrivial automorphism.

Remark 3.5 Any morphism $f: T \rightarrow T^{\prime}$ is uniquely determined by the inner edges $e=\{v, w\} \in E(T)$ that $f$ contracts $f(v)=f(w)$. Moreover, given a planted planar tree with leaves $T$ and an inner edge $e=\{v, w\} \in E(T)$ the quotient tree $T / e$, obtained by contracting $e$ to a vertex $[e] \in V(T / e)$, carries a unique structure of planted planar tree with leaves such that the natural projection $p_{e}^{T}: T \rightarrow T / e$ is a morphism in PPTL; see Figure 4. This morphism induces identifications

$$
V(T) \backslash\{v, w\}=V(T / e) \backslash\{[e]\}, \quad E(T) \backslash\{e\}=E(T / e) .
$$

One can similarly define a morphism $p_{K}^{T}: T \rightarrow T / K$ in PPTL contracting the connected components of any subcomplex $K \subset T$ formed by inner edges; see Figure 14 below for a more complicated example. 


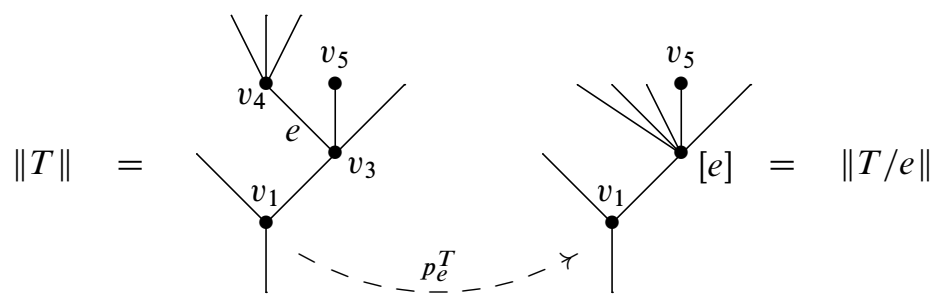

Figure 4: The morphism $p_{e}^{T}: T \rightarrow T / e$ in PPTL contracting the inner edge $e=\left\{v_{3}, v_{4}\right\}$

Definition 3.6 Given a planted planar tree $T$ with $n$ leaves and $n$ planted planar trees with leaves $T_{1}, \ldots, T_{n}$, we denote by $T\left(T_{1}, \ldots, T_{n}\right)$ the planted planar tree with the same root as $T$, the leaves are the disjoint union of the leaves of all $T_{i}$, and the space $\left\|T\left(T_{1}, \ldots, T_{n}\right)\right\|$ is obtained by grafting the root edge of $\left\|T_{i}\right\|$ in the $i$-th leaf edge of $\|T\|$ with respect to the path order $\preceq$ in $L(T) \subset V(T), 1 \leq i \leq n$; see Figure 5 .

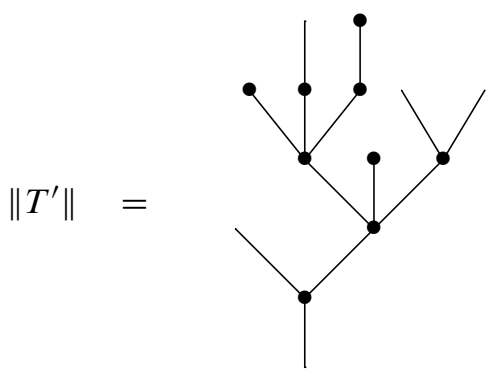

Figure 5: The grafting $T^{\prime}=T\left(U, C_{0}, C_{1}, C_{1}\left(C_{0}\right), C_{2}\right)$ for $T$ in Figure 2

Grafting is associative, ie

$$
\begin{aligned}
T\left(T_{1}\left(T_{1,1}, \ldots, T_{1, p_{1}}\right), \ldots,\right. & \left.T_{n}\left(T_{n, 1}, \ldots, T_{n, p_{n}}\right)\right) \\
= & \left(T\left(T_{1}, \ldots, T_{n}\right)\right)\left(T_{1,1}, \ldots, T_{1, p_{1}}, \ldots, T_{n, 1}, \ldots, T_{n, p_{n}}\right) .
\end{aligned}
$$

The planted planar tree $U$ with only one edge and one leaf, $\|U\|=\mid$, is a unit for the grafting operation:

$$
U(T)=T, \quad T(U, \ldots, U)=T .
$$

The category PPTL splits as the coproduct of the full subcategories PPTL $(n)$ of trees with $n$ leaves:

$$
\text { PPTL }=\coprod_{n \geq 0} \operatorname{PPTL}(n) .
$$


Notice that the grafting operation is functorial in PPTL in the sense of the following obvious lemma.

Lemma 3.7 The sequence $\{\mathbf{P P T L}(n)\}_{n \geq 0}$ with the grafting operation and the unit $U$ is an operad in the cartesian closed category of small categories.

Lemma 3.8 All planted planar trees with leaves can be obtained by grafting corollas and $U$.

Proof By induction on the height of the planted planar tree withe leaves $T$. On the one hand, if $\operatorname{ht}(T)=1$ then $T=U$ or $C_{0}$. On the other hand, any $T \neq U, C_{0}$ can be decomposed as $T=C_{n}\left(T_{1}, \ldots, T_{n}\right)$ where $n+1$ is the degree of the unique level 1 vertex of $T$ and $\operatorname{ht}\left(T_{i}\right)<\operatorname{ht}(T), 1 \leq i \leq n$.

For instance, $T$ in Figure 2 is

$$
T=C_{2}\left(U, C_{3}\left(C_{3}, C_{0}, U\right)\right)=C_{2} \circ_{2}\left(\left(C_{3} \circ_{2} C_{0}\right) \circ_{1} C_{3}\right) .
$$

Definition 3.9 An operadic functor with values in $\mathscr{V}$ is a functor

$$
\mathcal{G}: \text { PPTL } \longrightarrow \mathscr{V}
$$

equipped with a unit morphism $u: \mathbb{I} \rightarrow \mathcal{G}\left(C_{1}\right)$ and natural isomorphisms

$$
\mathcal{G}\left(T\left(T_{1}, \ldots, T_{n}\right)\right) \cong \mathcal{G}(T) \otimes \mathcal{G}\left(T_{1}\right) \otimes \cdots \otimes \mathcal{G}\left(T_{n}\right)
$$

that we call grafting isomorphisms, such that:

- $\mathcal{G}(U)=\mathbb{I}$.

- The following composition of grafting isomorphisms is a coherent composition of associativity and symmetry constraints in $\mathscr{V}$ :

$\mathcal{G}(T) \otimes \mathcal{G}\left(T_{1}\right) \otimes \mathcal{G}\left(T_{1,1}\right) \otimes \cdots \otimes \mathcal{G}\left(T_{1, p_{1}}\right) \otimes \cdots \otimes \mathcal{G}\left(T_{n}\right) \otimes \mathcal{G}\left(T_{n, 1}\right) \otimes \cdots \otimes \mathcal{G}\left(T_{n, p_{n}}\right)$ $\cong \mathcal{G}(T) \otimes \mathcal{G}\left(T_{1}\left(T_{1,1}, \ldots, T_{1, p_{1}}\right)\right) \otimes \cdots \otimes \mathcal{G}\left(T_{n}\left(T_{n, 1}, \ldots, T_{n, p_{n}}\right)\right)$

$\cong \mathcal{G}\left(T\left(T_{1}\left(T_{1,1}, \ldots, T_{1, p_{1}}\right), \ldots, T_{n}\left(T_{n, 1}, \ldots, T_{n, p_{n}}\right)\right)\right)$

$=\mathcal{G}\left(\left(T\left(T_{1}, \ldots, T_{n}\right)\right)\left(T_{1,1}, \ldots, T_{1, p_{1}}, \ldots, T_{n, 1}, \ldots, T_{n, p_{n}}\right)\right)$

$\cong \mathcal{G}\left(T\left(T_{1}, \ldots, T_{n}\right)\right) \otimes \mathcal{G}\left(T_{1,1}\right) \otimes \cdots \otimes \mathcal{G}\left(T_{1, p_{1}}\right) \otimes \cdots \otimes \mathcal{G}\left(T_{n, 1}\right) \otimes \cdots \otimes \mathcal{G}\left(T_{n, p_{n}}\right)$ $\cong \mathcal{G}(T) \otimes \mathcal{G}\left(T_{1}\right) \otimes \cdots \otimes \mathcal{G}\left(T_{n}\right) \otimes \mathcal{G}\left(T_{1,1}\right) \otimes \cdots \otimes \mathcal{G}\left(T_{1, p_{1}}\right)$

$$
\otimes \cdots \otimes \mathcal{G}\left(T_{n, 1}\right) \otimes \cdots \otimes \mathcal{G}\left(T_{n, p_{n}}\right) .
$$


- The following grafting isomorphisms are (compositions of) unit constraints in $\mathscr{V}$ :

$$
\mathbb{I} \otimes \mathcal{G}(T)=\mathcal{G}(U) \otimes \mathcal{G}(T) \underset{\text { grafting }}{\cong} \mathcal{G}(U(T))=\mathcal{G}(T),
$$

$\mathcal{G}\left(T^{\prime}\right) \otimes \mathbb{I} \otimes \cdots \otimes \mathbb{I}=\mathcal{G}\left(T^{\prime}\right) \otimes \mathcal{G}(U) \otimes \cdots \otimes \mathcal{G}(U) \underset{\text { grafting }}{\stackrel{\cong}{\longrightarrow}} \mathcal{G}(T(U, \ldots, U))=\mathcal{G}(T)$.

- Suppose $T^{\prime}=C_{1}(T)$; see Figure 6. Let $f: T^{\prime} \rightarrow T$ be the morphism which

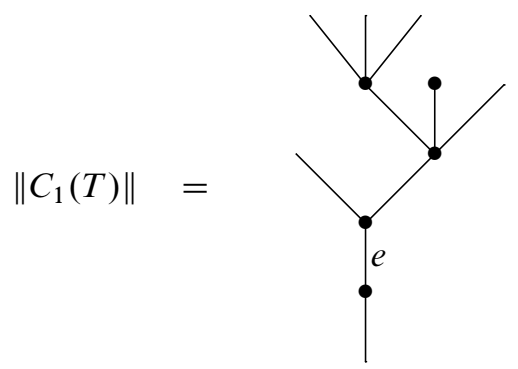

Figure 6: The planted planar tree with leaves $T^{\prime}=C_{1}(T)$ for $T$ as in

Figure 2. Here we denote $e$ the incoming edge of the level 1 vertex of $T^{\prime}$.

contracts the incoming edge of the level 1 vertex of $T^{\prime}$. Then the following morphism is the left unit constraint in $\mathscr{V}$ :

$$
\mathbb{I} \otimes \mathcal{G}(T) \stackrel{u \otimes \mathrm{id}}{\longrightarrow} \mathcal{G}\left(C_{1}\right) \otimes \mathcal{G}(T) \underset{\text { grafting }}{\stackrel{G}{\longrightarrow}} \mathcal{G}\left(T^{\prime}\right) \stackrel{\mathcal{G}(f)}{\longrightarrow} \mathcal{G}(T) .
$$

- Suppose $T^{\prime}=T\left(C_{1}, \ldots, C_{1}\right)$; see Figure 7. Let $f: T^{\prime} \rightarrow T$ be the morphism

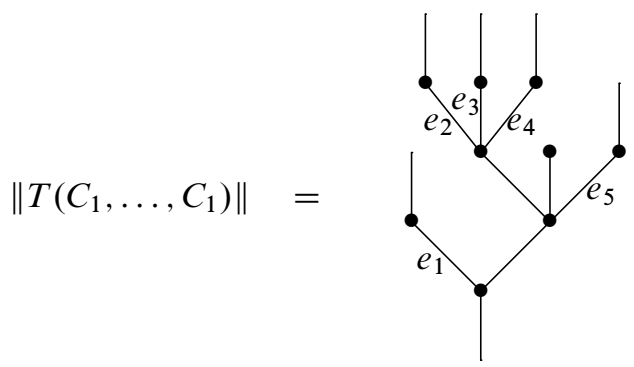

Figure 7: The planted planar tree with leaves $T^{\prime}=T\left(C_{1}, \ldots, C_{1}\right)$ for $T$ as in Figure 2. Here $e_{i}$ denotes the inner edges adjacent to the leaf edges in $T^{\prime}$.

which contracts all the inner edges adjacent to the leaf edges in $T^{\prime}$. Then the following morphism is a composition of right unit constraints in $\mathscr{V}$ :

$\mathcal{G}(T) \otimes \mathbb{I} \otimes \cdots \otimes \mathbb{I} \stackrel{\text { id } \otimes u \otimes \cdots \otimes u}{\longrightarrow} \mathcal{G}(T) \otimes \mathcal{G}\left(C_{1}\right) \otimes \cdots \otimes \mathcal{G}\left(C_{1}\right) \underset{\text { grafting }}{\stackrel{\cong}{\longrightarrow}} \mathcal{G}\left(T^{\prime}\right) \stackrel{\mathcal{G}(f)}{\longrightarrow} \mathcal{G}(T)$. 
A morphism of operadic functors $\varphi: \mathcal{G} \rightarrow \mathcal{H}$ is a natural transformation compatible with the grafting isomorphisms, with the unit morphism, and such that $\varphi(U)=\mathrm{id}_{\mathbb{I}}$.

The following equivalence between operads and operadic functors was sketched by Ginzburg and Kapranov in the symmetric case [10, 1.2].

Proposition 3.10 There is an equivalence between the categories of operads in $\mathscr{V}$ and operadic functors with values in $\mathscr{V}$.

Proof Denote OpFunc $(\mathscr{V})$ the category of operadic functors with values in $\mathscr{V}$. We are going to define adjoint equivalences

$$
\mathrm{Op}(\mathscr{V}) \underset{R}{\stackrel{L}{\rightleftarrows}} \text { OpFunc( } \mathscr{V})
$$

Given an operadic functor $\mathcal{G}$ we set

$$
R(\mathcal{G})(n)=\mathcal{G}\left(C_{n}\right),
$$

the unit of the operad $R(\mathcal{G})$ is $u: \mathbb{I} \rightarrow \mathcal{G}\left(C_{1}\right)=R(\mathcal{G})(1)$, and multiplications in $R(\mathcal{G})$ are defined by the morphisms

$$
f_{n ; p_{1}, \ldots, p_{n}}: C_{n}\left(C_{p_{1}}, \ldots, C_{p_{n}}\right) \longrightarrow C_{p_{1}+\cdots+p_{n}}
$$

which contract all inner edges:

$$
\begin{aligned}
& R(\mathcal{G})(n) \otimes R(\mathcal{G})\left(p_{1}\right) \otimes \cdots \otimes R(\mathcal{G})\left(p_{n}\right)=\mathcal{G}\left(C_{n}\right) \otimes \mathcal{G}\left(C_{p_{1}}\right) \otimes \cdots \otimes \mathcal{G}\left(C_{p_{n}}\right) \\
& \begin{array}{c}
\mu_{n ; p_{1}, \ldots, p_{n}} \mid \\
\mathcal{G}\left(C_{n}\left(C_{p_{1}}, \ldots, C_{p_{n}}\right)\right) \\
\downarrow \mathcal{G}\left(f_{n ; p_{1}, \ldots, p_{n}}\right) \\
R(\mathcal{G})\left(p_{1}+\cdots+p_{n}\right) \rightleftharpoons \mathcal{G}\left(C_{p_{1}+\cdots+p_{n}}\right)
\end{array}
\end{aligned}
$$

Conversely, if $\mathcal{O}$ is an operad then the corresponding operadic functor $L(\mathcal{O})$ is defined on objects as

$$
L(\mathcal{O})(T)=\bigotimes_{u \in I(T)} \mathcal{O}(\tilde{u})
$$

(see Figure 8). The morphism $u: \mathbb{I} \rightarrow \mathcal{O}(1)=L(\mathcal{O})\left(C_{1}\right)$ is the unit of the operad. Grafting isomorphisms are coherent compositions of associativity and symmetry constraints in $\mathscr{V}$. Moreover, let $T$ be a planted planar tree with leaves and $e=\{v, w\} \in E(T)$ 


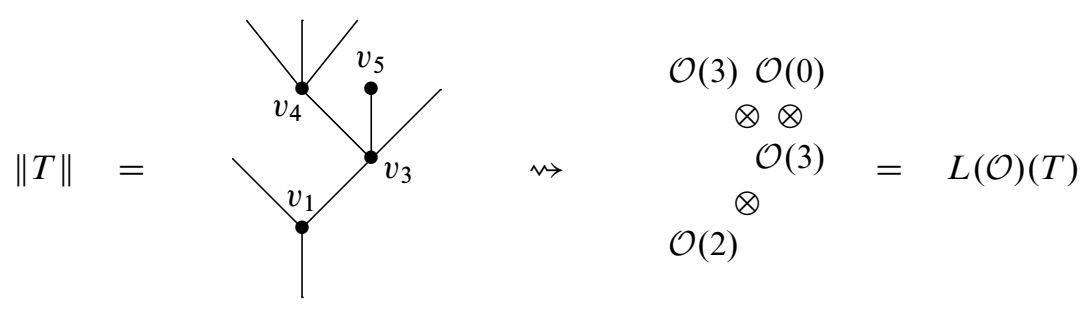

Figure 8: The object $L(\mathcal{O})(T)$ associated to the planted planar tree with leaves $T$ in Figure 2

an inner edge which is the $i$-th incoming edge of $v$. The morphism induced by the natural projection $p_{e}^{T}: T \rightarrow T / e$ in Remark 3.5 is

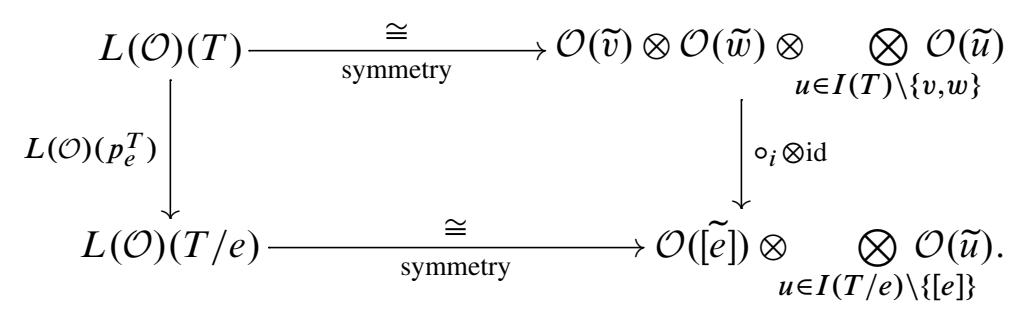

Here we use that $[\tilde{e}]=\tilde{v}+\widetilde{w}-1$; see Figure 9.

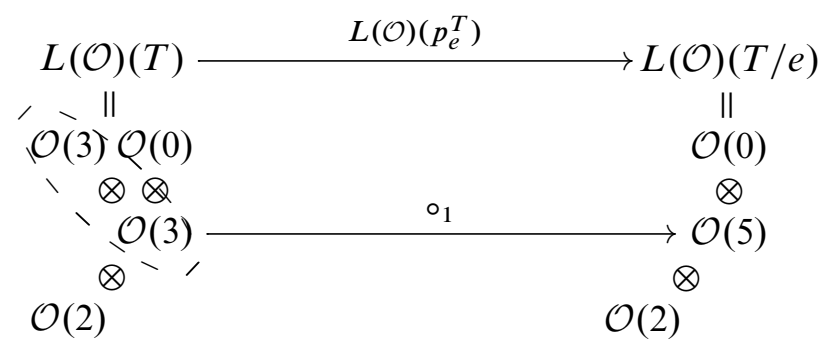

Figure 9: The morphism $L(\mathcal{O})\left(p_{e}^{T}\right)$ for $T$ and $e=\left\{v_{3}, v_{4}\right\}$ as in Figure $4-$ see also Figure 8

The unit natural transformation $\mathcal{O} \rightarrow R L(\mathcal{O})$ is the identity morphism, and the counit $\varepsilon: L R(\mathcal{G}) \rightarrow \mathcal{G}$ is defined by grafting isomorphisms:

$$
\varepsilon(T): L R(\mathcal{G})(T)=\underset{u \in I(T)}{\bigotimes} \mathcal{G}\left(C_{\widetilde{u}}\right) \underset{\text { grafting }}{\cong} \mathcal{G}(T) .
$$

Here we use that any planted planar tree with leaves $T$ can be obtained by grafting appropriately the corollas $C_{\widetilde{u}}, u \in I(T)$; compare the previous lemma. 
Examples of planted planar trees with leaves illustrating relations (1) and (2) in Remark 2.6 are depicted in Figure 10.
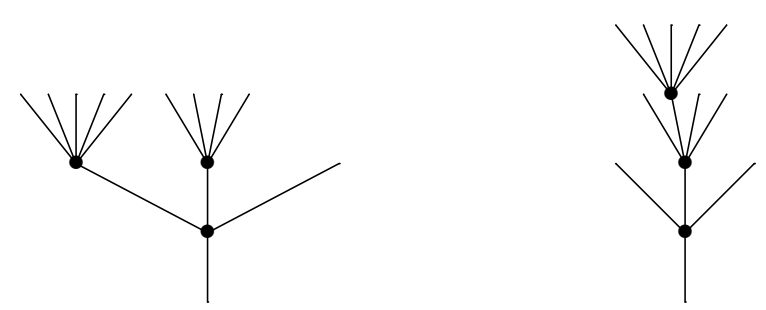

Figure 10: The planted planar trees with leaves illustrating the associativity relations $\left(C_{3} \circ_{2} C_{4}\right) \circ_{1} C_{5}=\left(C_{3} \circ_{1} C_{5}\right) \circ_{6} C_{4}$ in Remark 2.6 (1) and $\left(C_{3} \circ_{2} C_{4}\right) \circ_{3} C_{5}=C_{3} \circ_{2}\left(C_{4} \circ_{2} C_{5}\right)$ in Remark 2.6 (2), respectively

\section{The monoidal category of morphisms}

The category Mor $(\mathscr{C})$ of morphisms in $\mathscr{C}$ can be regarded as the category of functors $\mathbf{2} \rightarrow \mathscr{C}$, where $\mathbf{2}$ is the category with two objects, 0 and 1 , and only one nonidentity morphism $0 \rightarrow 1$, ie it is the poset $\{0<1\}$. A morphism $f: U \rightarrow V$ in $\mathscr{C}$ is identified with the functor $f: \mathbf{2} \rightarrow \mathscr{C}$ defined by $f(0)=U, f(1)=V$ and $f(0 \rightarrow 1)=f$.

The category Mor $(\mathscr{C})$ carries a biclosed monoidal structure given by the $\odot$ product of morphisms $f \odot g$ :

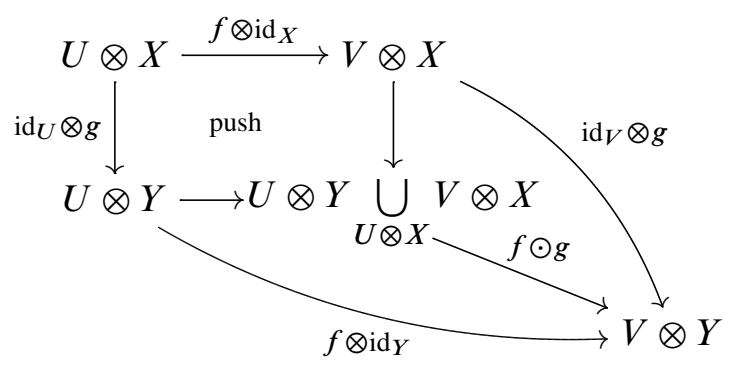

This monoidal structure is symmetric provided $\otimes$ is. If 0 denotes the initial object of $\mathscr{b}$, the functor

$$
\begin{aligned}
& \mathscr{b} \longrightarrow \operatorname{Mor}(\mathscr{C}), \\
& X \mapsto(0 \rightarrow X),
\end{aligned}
$$

is strong (symmetric) monoidal. We regard $\mathscr{C}$ as a full subcategory of $\operatorname{Mor}(\mathscr{C})$ through this functor. 
Notice that pushouts in $\mathscr{C}$ are a special kind of morphism in Mor( $\mathscr{C})$. The following lemma asserts that the $\odot$ product preserves pushouts in $\mathscr{C}$.

Lemma 4.1 Given two pushout diagrams in $\mathscr{C}, i=1,2$,

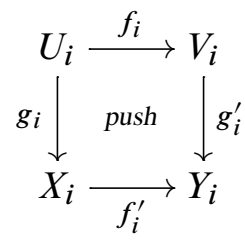

the following diagram in $\mathscr{b}$ is also a pushout:

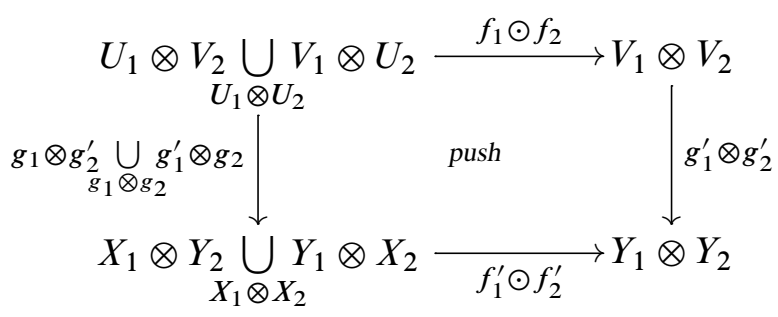

This lemma follows straightforwardly from the very definition of $\odot$ together with the fact that $\otimes$ is biclosed, and hence it preserves colimits in both variables.

Definition 4.2 The category $\mathscr{b}$ is a monoidal model category if it is endowed with a model structure satisfying the pushout product axiom:

- Let $f$ and $g$ be cofibrations in $\mathscr{C}$. The morphism $f \odot g$ is also a cofibration. If in addition $f$ or $g$ is a weak equivalence, then so is $f \odot g$.

This axiom was considered by Schwede and Shipley [20, Definition 3.1] for $\mathscr{C}$ symmetric, but it also makes sense in the nonsymmetric case. Actually, following the terminology of Meyer [18] and Batanin [2, Definition 2.2], which work in a nonsymmetric context, the first half of the pushout product axiom says that all cofibrations in $\mathscr{b}$ are closed.

Remark 4.3 The pushout product axiom implies that the tensor product of cofibrant objects is cofibrant. Moreover, if $X$ is a cofibrant object and $f$ is a (trivial) cofibration in $\mathscr{C}$ then $X \otimes f$ and $f \otimes X$ are (trivial) cofibrations. In particular, by Ken Brown's lemma [15, Lemma 1.1.12], for $X$ cofibrant the functors $X \otimes-$ and $-\otimes X$ preserve weak equivalences between cofibrant objects. Furthermore, if $f$ and $g$ are (trivial) cofibrations with cofibrant source, then so is $f \odot g$. 
Lemma 4.4 Let $\mathscr{C}$ be a left proper monoidal model category. Consider two commutative squares in Mor( $(\mathscr{C})$ where the rows are cofibrations and the columns are weak equivalences between cofibrant objects, $i=1,2$,

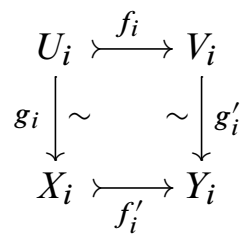

Then in the following diagram the rows are also cofibrations and the columns are weak equivalences between cofibrant objects:

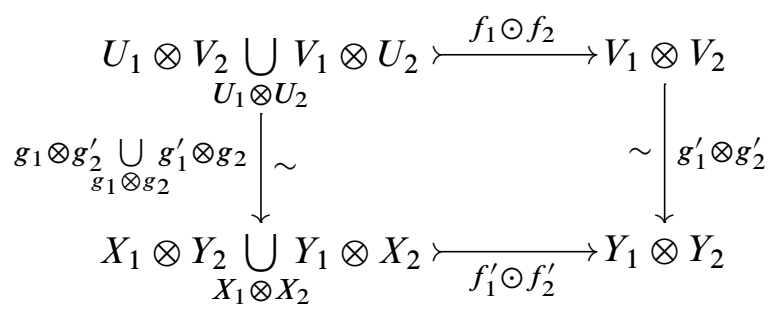

Proof Looking at Definition 4.2 and the remark afterwards we notice that it is only left to check that the left column is a weak equivalence. This follows easily from the gluing property in left proper model categories [14, Proposition 13.5.4].

Given morphisms $f_{i}: U_{i} \rightarrow V_{i}$ in $\mathscr{b}, 1 \leq i \leq n$, the target of $f_{1} \odot \cdots \odot f_{n}$ is the iterated tensor product of the targets $V_{1} \otimes \cdots \otimes V_{n}$. This object is the colimit of the diagram

$$
f_{1} \otimes \cdots \otimes f_{n}: \mathbf{2}^{n} \longrightarrow \mathscr{C},
$$

since $2^{n}$ has a final object $(1, \ldots, ., 1)$. The source of $f_{1} \odot \cdots \odot f_{n}$ is the colimit of the restriction of this diagram to the full subcategory of $2^{n}$ obtained by removing the final object. For simplicity, we denote it by $s\left(f_{1} \odot \cdots \odot f_{n}\right)$ :

$$
f_{1} \odot \cdots \odot f_{n}: s\left(f_{1} \odot \cdots \odot f_{n}\right) \longrightarrow V_{1} \otimes \cdots \otimes V_{n} .
$$

The universal property of $s\left(f_{1} \odot \cdots \odot f_{n}\right)$ in $\mathscr{C}$ refers to canonical morphisms

$$
\kappa_{i}: V_{1} \otimes \cdots \otimes V_{i-1} \otimes U_{i} \otimes V_{i+1} \otimes \cdots \otimes V_{n} \longrightarrow s\left(f_{1} \odot \cdots \odot f_{n}\right), \quad 1 \leq i \leq n,
$$

with $\left(f_{1} \odot \cdots \odot f_{n}\right) \kappa_{i}=\mathrm{id}^{\otimes(i-1)} \otimes f_{i} \otimes \mathrm{id}^{\otimes(n-i)}$. Any collection of morphisms

$$
g_{i}: V_{1} \otimes \cdots \otimes V_{i-1} \otimes U_{i} \otimes V_{i+1} \otimes \cdots \otimes V_{n} \longrightarrow X, \quad 1 \leq i \leq n,
$$


such that the following squares commute, $1 \leq i<j \leq n$,

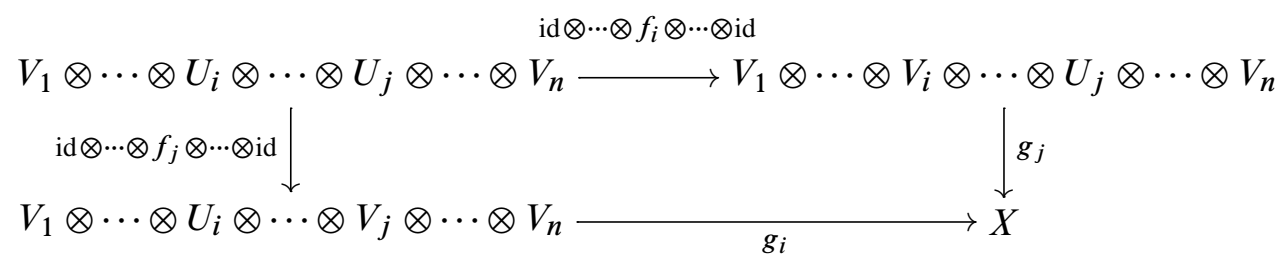

induces a unique morphism $g: s\left(f_{1} \odot \cdots \odot f_{n}\right) \rightarrow X$ such that $g_{i}=g \kappa_{i}, 1 \leq i \leq n$. Compare the paragraph preceding [2, Lemma 2.2].

\section{The relevant operad pushout}

The forgetful functor from operads to sequences $\mathrm{Op}(\mathscr{V}) \rightarrow \mathscr{V}^{\mathbb{N}}$ has a left adjoint $\mathcal{F}: \mathscr{V}^{\mathbb{N}} \rightarrow \mathrm{Op}(\mathscr{V})$, the free operad functor, explicitly constructed for example in [3, Appendix B]. An alternative construction in terms of trees is as follows (see [2, Section 3]):

$$
\mathcal{F}(V)(n)=\coprod_{T} \bigotimes_{v \in I(T)} V(\widetilde{v})
$$

where $T$ runs over a set of isomorphism classes of trees with $n$ leaves in PPTL. The product $o_{i}, 1 \leq i \leq m$,

$$
\begin{array}{rlrl}
\mathcal{F}(V)(m) \otimes \mathcal{F}(V)(n) & =\left(\coprod_{T^{\prime}} \bigotimes_{u \in I\left(T^{\prime}\right)} V(\widetilde{u})\right) \otimes\left(\coprod_{T} \bigotimes_{v \in I(T)} V(\widetilde{v})\right) \begin{array}{l}
\operatorname{card} L\left(T^{\prime}\right)=m \\
\operatorname{card} L(T)=n
\end{array} \\
& \cong \coprod_{T^{\prime}, T}\left(\bigotimes_{u \in I\left(T^{\prime}\right)} V(\tilde{u}) \otimes \bigotimes_{v \in I(T)} V(\widetilde{v})\right) & \\
\mathcal{F}(V)(m+n-1) & =\bigsqcup_{T^{\prime \prime}} \bigotimes_{w \in I\left(T^{\prime \prime}\right)} V(\widetilde{w}) & \operatorname{card} L\left(T^{\prime \prime}\right)=m+n-1
\end{array}
$$

sends the factor corresponding to the trees $T$ and $T^{\prime}$ in the source to the factor of $T^{\prime \prime}=T^{\prime} \circ_{i} T$ in the target:

$$
I\left(T^{\prime} \circ_{i} T\right)=I\left(T^{\prime}\right) \sqcup I(T), \quad \bigotimes_{u \in I\left(T^{\prime}\right)} V(\tilde{u}) \otimes \bigotimes_{v \in I(T)} V(\tilde{v})=\bigotimes_{w \in I\left(T^{\prime} \circ_{i} T\right)} V(\tilde{w}) .
$$

The unit $u: \mathbb{I} \rightarrow \mathcal{F}(V)(1)$ is the inclusion of the factor of the coproduct corresponding to the tree with one leaf a no inner vertex, ie the unit of the grafting operation. 
The unit of the adjunction $V \rightarrow \mathcal{F}(V)$ in $\mathscr{V} \mathbb{N}$ is given by the morphisms in $\mathscr{V}, n \geq 0$,

$$
V(n) \stackrel{\begin{array}{c}
\text { inclusion of the factor } \\
\text { corresponding to } C_{n}
\end{array}}{\longrightarrow} \mathcal{F}(V)(n) \text {. }
$$

Given an operad $\mathcal{O}$ with associated operadic functor $L(\mathcal{O})$, if we denote $p_{T}: T \rightarrow C_{n}$ the morphism in PPTL collapsing all inner edges of a tree $T$ with $n$ leaves, then the counit $\mathcal{F}(\mathcal{O}) \rightarrow \mathcal{O}$ is defined by the following morphisms, $n \geq 0$,

$$
\mathcal{F}(\mathcal{O})(n)=\bigsqcup_{T} \bigotimes_{v \in I(T)} \mathcal{O}(\widetilde{v})=\coprod_{T} L(\mathcal{O})(T) \stackrel{\left(L(\mathcal{O})\left(p_{T}\right)\right)_{T}}{\longrightarrow} L(\mathcal{O})\left(C_{n}\right)=\mathcal{O}(n) .
$$

An analogous construction for symmetric operads was considered by Ginzburg and Kapranov in [10, 2.1].

In this section we give an explicit construction of the pushout of two morphisms in $\mathrm{Op}(\mathscr{V})$ as follows:

$$
\mathcal{O} \longleftarrow \stackrel{g}{\mathcal{F}}(U) \stackrel{\mathcal{F}(f)}{\longrightarrow} \mathcal{F}(V)
$$

Consider the adjoint diagram in $\mathscr{V}^{\mathbb{N}}$ :

$$
\mathcal{O} \stackrel{\bar{g}}{\longleftarrow} U \stackrel{f}{\longrightarrow} V
$$

The pushout of Equation (2) is an operad $\mathcal{P}$ together with morphisms $f^{\prime}: \mathcal{O} \rightarrow \mathcal{P}$ in $\mathrm{Op}(\mathcal{V})$ and $\bar{g}^{\prime}: V \rightarrow \mathcal{P}$ in $\mathscr{V}^{\mathbb{N}}$ such that $f^{\prime} \bar{g}=\bar{g}^{\prime} f$ in $\mathscr{V}^{\mathbb{N}}$. Moreover, given an operad $\mathcal{P}^{\prime}$ and morphisms $f^{\prime \prime}: \mathcal{O} \rightarrow \mathcal{P}^{\prime}$ in $\operatorname{Op}(\mathscr{V})$ and $\bar{g}^{\prime \prime}: V \rightarrow \mathcal{P}^{\prime}$ in $\mathscr{V} \mathbb{N}$ with $f^{\prime \prime} \bar{g}=\bar{g}^{\prime \prime} f$ in $\mathscr{V} \mathbb{N}$, there is a unique morphism $h: \mathcal{P} \rightarrow \mathcal{P}^{\prime}$ in $\operatorname{Op}(\mathscr{V})$ such that $f^{\prime \prime}=h f^{\prime}$ and $\bar{g}^{\prime \prime}=h \bar{g}^{\prime}$ in $\mathscr{V}^{\mathbb{N}}$.

Given a planted planar tree with leaves $T$ we denote

$$
\begin{aligned}
V^{e}(T) & =\{v \in V(T) ; \text { level }(v) \text { is even }\}, & & V^{o}(T)=V(T) \backslash V^{e}(T), \\
I^{e}(T) & =I(T) \cap V^{e}(T), & I^{o}(T) & =I(T) \cap V^{o}(T)
\end{aligned}
$$

(see Figure 11). From now on, we will only consider one tree in each isomorphism class of objects in PPTL.

The idea behind our construction of the pushout of Equation (2) is as follows. For any planted planar tree with leaves concentrated in even levels, such as $T$ in Figure 11, we replace any inner even (resp. odd) vertex $v$ with the piece of $V$ (resp. $\mathcal{O})$ in degree $\widetilde{v}$, 

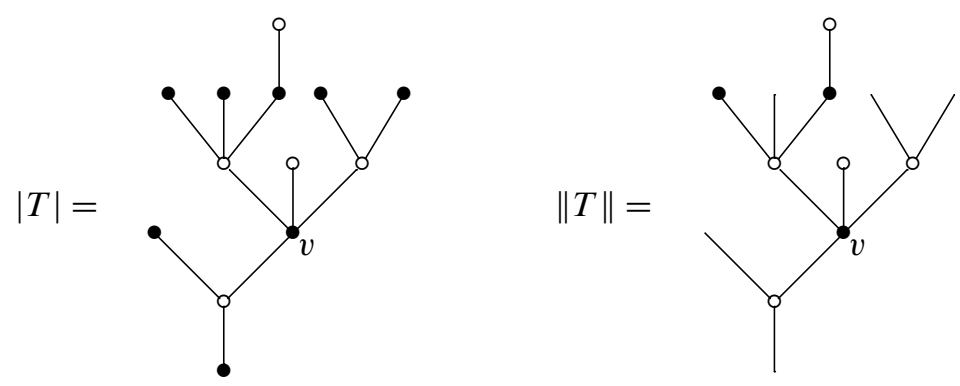

Figure 11: For a planted planar tree with leaves $T$, on the left (resp. right) we denote $\circ$ the vertices in $V^{o}(T)$ (resp. $\left.I^{o}(T)\right)$ and $\bullet$ the vertices in $V^{e}(T)$ (resp. in $\left.I^{e}(T)\right)$.

and transform adjacency relations into tensor products.
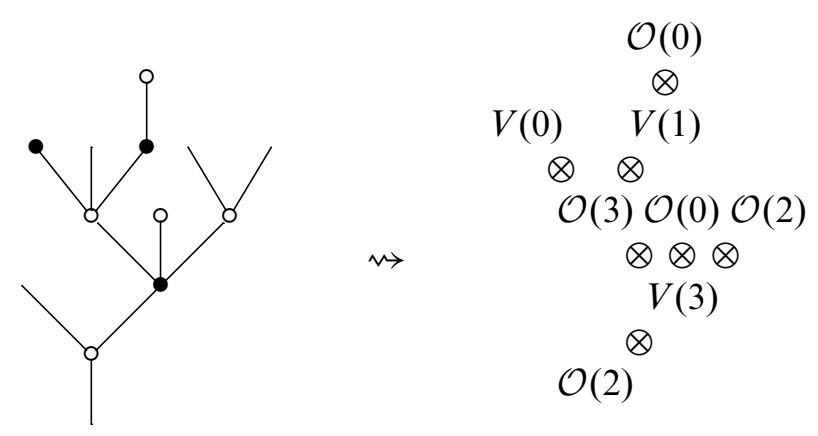

In order to simplify the exposition of this intuitive idea, let us allow ourselves to talk about elements of this object in $\mathscr{V}$. We want to attach to $\mathcal{O}$ the product of these elements in a coherent way. More precisely, if $T$ has $n$ leaves, we attach these elements to $\mathcal{O}(n)$. For this, we must proceed by induction on the number of inner even vertices and require that, for any even inner vertex $v$, the image of the morphism induced by $f(\widetilde{v})$,

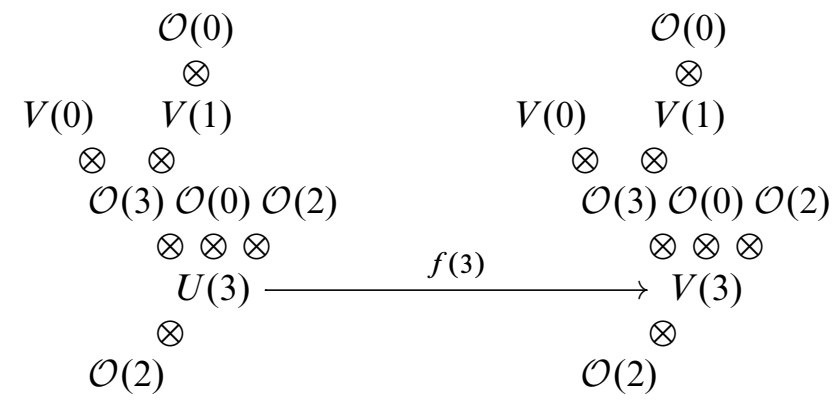


is attached according to the attachment of the tree $T^{\prime}$ with less even inner vertices obtained from $T$ by contracting the edges surrounding $v$ :
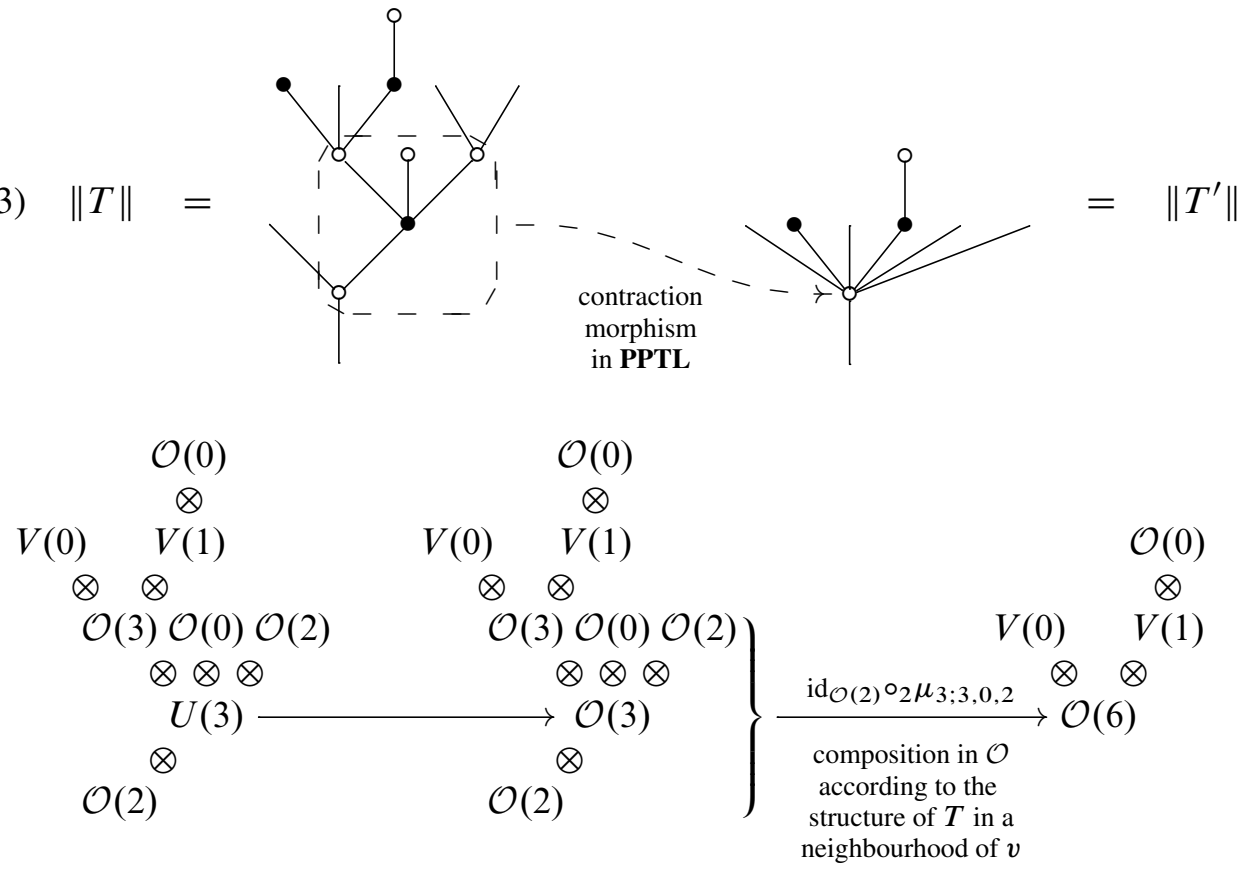

This inductive construction is carried out in the following lemma. In order to state it we need to introduce some terminology.

The star of a vertex $v \in V(T)$ is the subtree $\operatorname{St}(v) \subset T$ formed by the edges containing $v$, and the link $\operatorname{Lk}(v) \subset V(T)$ consists of the vertices adjacent to $v$; see Figure 12. When
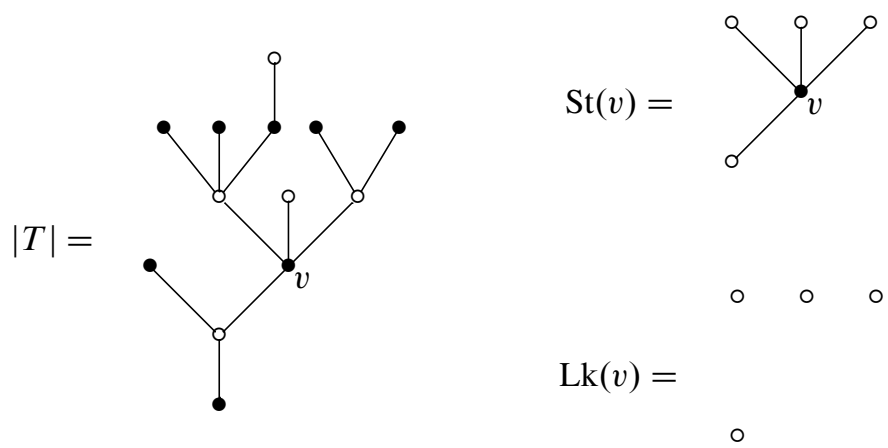

Figure 12: The star and the link of the vertex $v$ of the tree $T$ in Figure 11 
the star is formed by inner edges, the natural projection

$$
p_{\mathrm{St}(v)}^{T}: T \longrightarrow T / \mathrm{St}(v)
$$

is a morphism in PPTL; see Equation (3). This is the case if $v \in I^{e}(T)$ and $L(T) \subset$ $V^{e}(T)$. Moreover, in this case $p_{\mathrm{St}(v)}^{T}$ induces identifications

$$
I^{e}(T) \backslash\{v\}=I^{e}(T / \operatorname{St}(v)), \quad I^{o}(T) \backslash \operatorname{Lk}(v)=I^{o}(T / \operatorname{St}(v)) \backslash\{[\operatorname{St}(v)]\} .
$$

Furthermore, we will also consider the extended star $\overline{\operatorname{St}}(v) \subset T$, which is the planted planar tree with leaves whose inner part is $\operatorname{St}(v)$, the root edge is the outgoing edge of the minimum vertex $u \in \operatorname{Lk}(v)$, the leaves are the incoming edges of the vertices in $\operatorname{Lk}(v)$ except from $\{u, v\}$, and the planar order is the restriction of the planar order in $T$; see Figure 13. Notice that $\overline{\operatorname{St}}(v) / \operatorname{St}(v)=C_{r_{v}}$, where

$$
r_{v}=[\operatorname{St}(v)]=\operatorname{card} L(\overline{\operatorname{St}}(v))=\tilde{u}-1+\sum_{w \in \operatorname{Lk}(v) \backslash\{u\}} \tilde{w}=\left(\sum_{w \in \operatorname{Lk}(v)} \tilde{w}\right)-1 .
$$

The inductive construction of the pushout of Equation (2) is the in following scaring

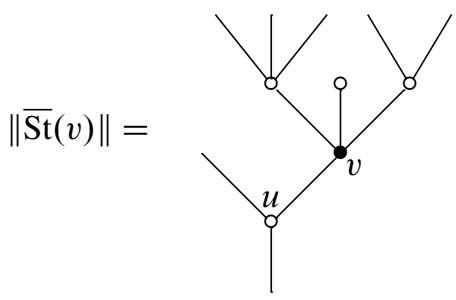

Figure 13: The extended star of the vertex $v$ of the planted planar tree with leaves $T$ in Figure 11 and Figure 12

lemma, whose statement is actually more complicated than its proof. For the sake of simplicity, from now on we use the same notation for an operad and for its associated operadic functor.

Lemma 5.1 There is a sequence of morphisms in $\mathscr{V} \mathbb{N}$,

$$
\mathcal{O}=P_{0} \stackrel{\varphi_{1}}{\longrightarrow} P_{1} \rightarrow \cdots \rightarrow P_{t-1} \stackrel{\varphi_{t}}{\longrightarrow} P_{t} \rightarrow \cdots,
$$

such that, for all $n \geq 0$, the morphism $\varphi_{t}(n): P_{t-1}(n) \rightarrow P_{t}(n)$ is the pushout of the following coproduct of morphisms indexed by the set of planted trees with $n$ leaves 
concentrated in even levels and $t$ inner even vertices, ie card $L(T)=n, L(T) \subset V^{e}(T)$, and $\operatorname{card} I^{e}(T)=t$,

$$
\coprod_{T} \bigodot_{v \in I^{e}(T)} f(\widetilde{v}) \otimes \bigotimes_{w \in I^{o}(T)} \mathcal{O}(\tilde{w})
$$

along the unique morphism

$$
\left(\psi_{t}^{T}\right)_{T}: \coprod_{T} s\left(\bigodot_{v \in I^{e}(T)} f(\tilde{v})\right) \otimes \bigotimes_{w \in I^{o}(T)} \mathcal{O}(\widetilde{w}) \longrightarrow P_{t-1}(n)
$$

such that, given $u \in I^{e}(T)$, for $t=1$ the morphism $\psi_{1}^{T}$ is

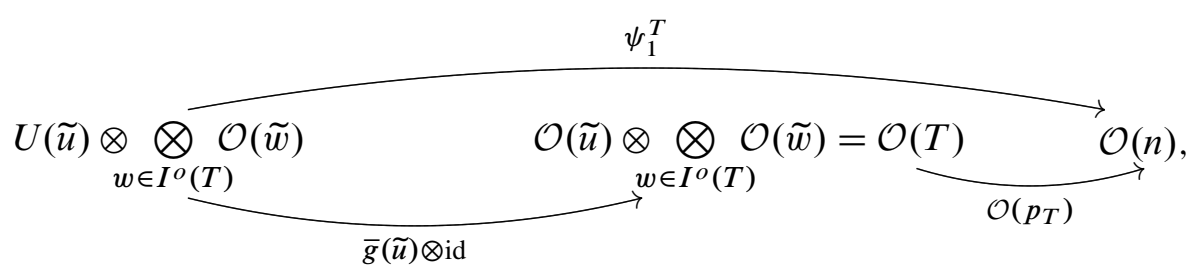

and for $t>1$ the composite morphism

$$
U(\tilde{u}) \otimes \otimes_{v \in I^{e}(T) \backslash\{u\}} V(\widetilde{v}) \otimes \bigotimes_{w \in I^{o}(T)} \mathcal{O}(\widetilde{w}) \stackrel{\kappa_{u} \otimes \mathrm{id}}{\longrightarrow} S\left(\bigodot_{v \in I^{e}(T)} f(\widetilde{v})\right) \underset{w \in I^{o}(T)}{\otimes} \mathcal{O}(\widetilde{w}) \stackrel{\psi_{t}^{T}}{\longrightarrow} P_{t-1}(n)
$$

coincides with the following composition that we call $\psi_{t, u}^{T}$ :

$$
\begin{aligned}
& U(\tilde{u}) \otimes \otimes V(\tilde{v}) \otimes \otimes \mathcal{O}(\tilde{w}) \\
& v \in I^{e}(T) \backslash\{u\} \quad w \in I^{o}(T) \\
& \bar{g}(\widetilde{u}) \otimes \mathrm{id} \downarrow \\
& \mathcal{O}(\tilde{u}) \otimes \otimes V(\widetilde{v}) \otimes \otimes \mathcal{O}(\tilde{w}) \\
& v \in I^{e}(T) \backslash\{u\} \quad w \in I^{o}(T) \\
& \cong \downarrow \text { symmetry } \\
& \otimes V(\tilde{v}) \otimes \otimes \mathcal{O}(\tilde{w}) \otimes \mathcal{O}(\overline{\operatorname{St}}(u)) \\
& v \in I^{e}(T) \backslash\{u\} \quad w \in I^{o}(T) \backslash \operatorname{Lk}(u) \\
& \operatorname{id} \otimes \mathcal{O}\left(p_{\overline{\mathrm{St}}(u)}\right) \downarrow \\
& \otimes V(\widetilde{v}) \otimes \otimes \mathcal{O}(\widetilde{w}) \otimes \mathcal{O}\left(r_{u}\right) \\
& v \in I^{e}(T / \operatorname{St}(u)) \quad w \in I^{o}(T / \operatorname{St}(u)) \backslash\{[\operatorname{St}(u)]\} \\
& \bar{\psi}_{t-1}^{T / \mathrm{St}(u)} \downarrow_{P_{t-1}(n)}
\end{aligned}
$$

Here $\left(\bar{\psi}_{t-1}^{T^{\prime}}\right)_{T^{\prime}}$ denotes the pushout of $\left(\psi_{t-1}^{T^{\prime}}\right)_{T^{\prime}}$, ie Equation (6) for $t-1$, along Equation (5). 
Proof The proof is by induction on $t \geq 0$. Notice that there is nothing to check for $t=0,1$. Let $t>1$ and assume everything works up to $t-1$. By the universal property of the source of an iterated $\odot$ product, described in Section 4, we only have to check the following compatibility condition: given two different vertices $u, u^{\prime} \in I^{e}(T)$, the following square commutes:

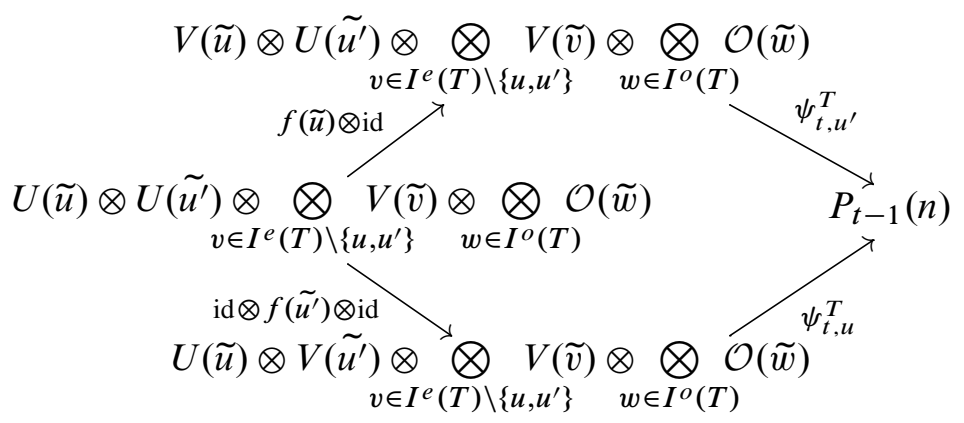

Here, for simplicity, we omit some symmetry isomorphisms in $\mathscr{V}$.

Denote $\operatorname{St}\left(u, u^{\prime}\right)=\operatorname{St}(u) \cup \operatorname{St}\left(u^{\prime}\right)$ and $\operatorname{Lk}\left(u, u^{\prime}\right)=\operatorname{Lk}(u) \cup \operatorname{Lk}\left(u^{\prime}\right)$. Suppose that $d\left(u, u^{\prime}\right)>2$. Then $\operatorname{St}(u) \cap \operatorname{St}\left(u^{\prime}\right)=\varnothing$ (see Figure 14), and moreover $t>2$. By induction hypothesis, in this case both compositions coincide with

$$
\begin{aligned}
& U(\tilde{u}) \otimes U\left(\tilde{u^{\prime}}\right) \otimes \otimes V(\tilde{v}) \otimes \otimes \mathcal{O}(\tilde{w}) \\
& v \in I^{e}(T) \backslash\left\{u, u^{\prime}\right\} \quad w \in I^{o}(T) \\
& \bar{g}(\tilde{u}) \otimes \bar{g}\left(\tilde{u^{\prime}}\right) \otimes \mathrm{id} \downarrow \\
& \mathcal{O}(\tilde{u}) \otimes \mathcal{O}\left(\tilde{u^{\prime}}\right) \otimes \otimes V(\tilde{v}) \otimes \otimes \mathcal{O}(\tilde{w}) \\
& v \in I^{e}(T) \backslash\left\{u, u^{\prime}\right\} \quad w \in I^{o}(T) \\
& \cong \downarrow \text { symmetry } \\
& v \in I^{e}(T) \backslash\left\{u, u^{\prime}\right\} \quad w \in I^{o}(T) \backslash \operatorname{Lk}\left(u, u^{\prime}\right) \\
& \downarrow \mathrm{id} \otimes \mathcal{O}\left(p_{\overline{\mathrm{St}}(u)}\right) \otimes \mathcal{O}\left(p_{\overline{\mathrm{St}}\left(u^{\prime}\right)}\right) \\
& \otimes V(\widetilde{v}) \quad \otimes \quad \otimes \mathcal{O}(\widetilde{w}) \quad \otimes \quad \mathcal{O}\left(r_{u}\right) \otimes \mathcal{O}\left(r_{u^{\prime}}\right) \\
& v \in I^{e}\left(T / \operatorname{St}\left(u, u^{\prime}\right)\right) \quad w \in I^{o}\left(T / \operatorname{St}\left(u, u^{\prime}\right)\right) \backslash\left\{[\operatorname{St}(u)],\left[\operatorname{St}\left(u^{\prime}\right)\right]\right\} \\
& \begin{array}{c}
\bar{\psi}_{t-2}^{T / \mathrm{St}\left(u, u^{\prime}\right)} \underset{P_{t-2}(n)}{\downarrow} \\
\varphi_{t-1}(n) \downarrow \\
P_{t-1}(n)
\end{array}
\end{aligned}
$$

See Figure 14 and Figure 15. 


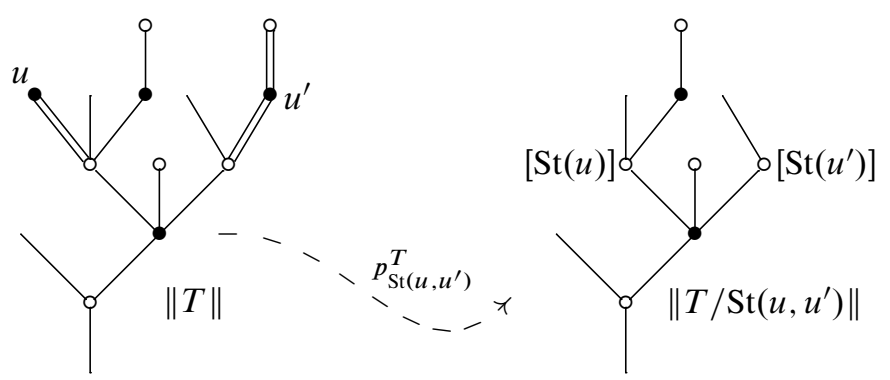

Figure 14: A planted planar tree $T$ with leaves in even levels and two even inner vertices $u$ and $u^{\prime}$ with $d\left(u, u^{\prime}\right)>2$. The disconnected subcomplex $\operatorname{St}\left(u, u^{\prime}\right) \subset T$ is in double lines. We illustrate the morphism $p_{\operatorname{St}\left(u, u^{\prime}\right)}^{T}$.

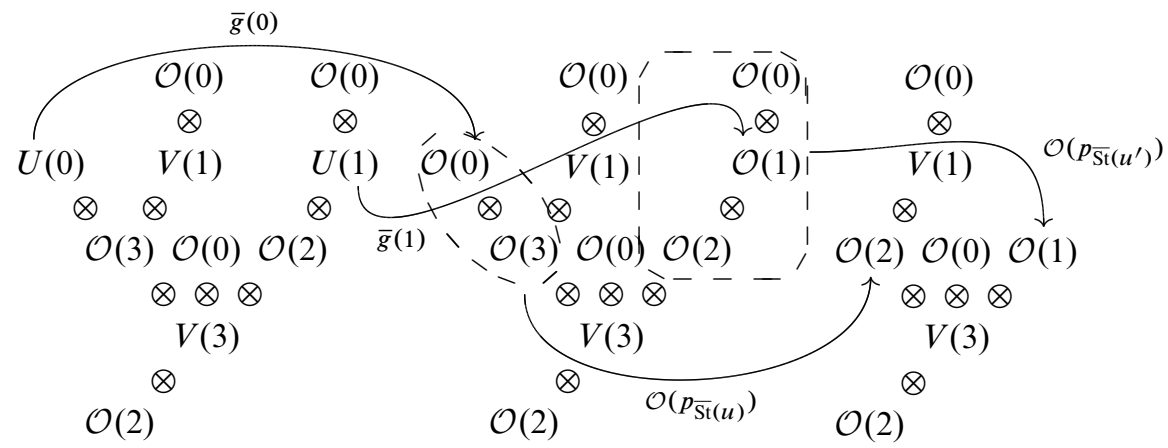

Figure 15: A sketch of Equation (7) for the planted planar tree $T$ with leaves in even levels and the two even inner vertices $u$ and $u^{\prime}$ in Figure 14

Suppose now that $d\left(u, u^{\prime}\right)=2$. Then the subcomplex $\operatorname{St}\left(u, u^{\prime}\right) \subset T$ is connected. Both factors share the unique vertex which is one step away from both $u$ and $u^{\prime}$; see Figure 16.
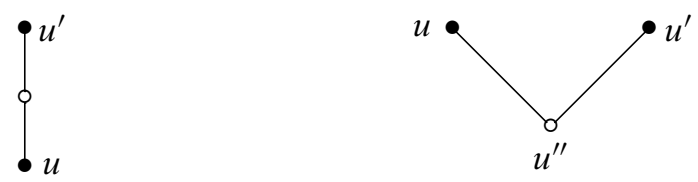

Figure 16: The only two possible relative positions of $u$ and $u^{\prime}, u<u^{\prime}$, within the planted planar tree with leaves $T$ if $d\left(u, u^{\prime}\right)=2$

Let $T^{\prime} \subset T$ be in this case the planted planar tree with leaves whose inner part is $\operatorname{St}\left(u, u^{\prime}\right)$, the root edge is the outgoing edge of the minimum vertex $u^{\prime \prime} \in \operatorname{Lk}\left(u, u^{\prime}\right)$, 
the leaves are the incoming edges of the vertices in $\operatorname{Lk}\left(u, u^{\prime}\right)$ not containing $u$ or $u^{\prime}$, and the planar order is the restriction of the planar order in $T$. This planted planar tree has $m$ leaves, where

$$
m=r_{u}+r_{u^{\prime}}-1,
$$

when the relative position of $u$ and $u^{\prime}$ is as in the first diagram of Figure 16; see also Figure 17. If the relative position is as in the second diagram of Figure 16, then (see Figure 18)

$$
m=\tilde{u^{\prime \prime}}+r_{u}+r_{u^{\prime}}-2
$$
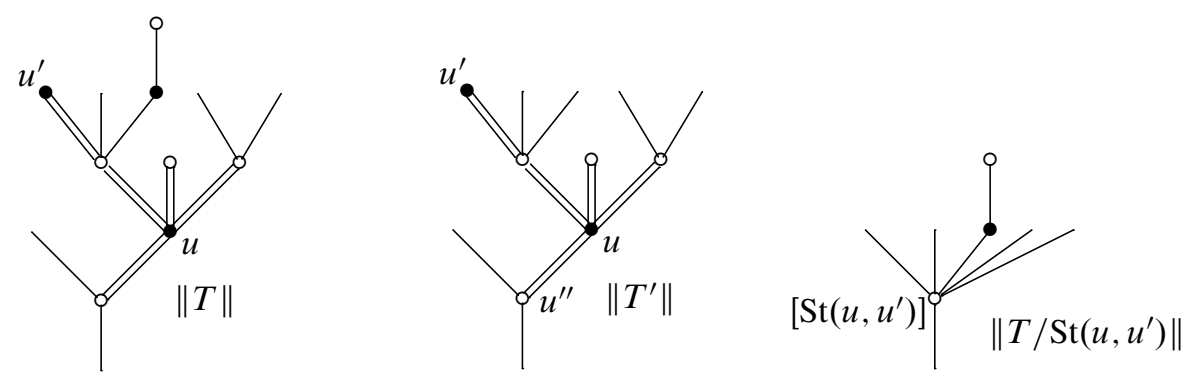

Figure 17: An example of the planted planar tree with leaves $T^{\prime}$ for the relative position of the vertices $u$ and $u^{\prime}$ as in the first diagram of Figure 16. The subcomplex $\operatorname{St}\left(u, u^{\prime}\right) \subset T$ is in double lines. We also depict $T / \operatorname{St}\left(u, u^{\prime}\right)$.
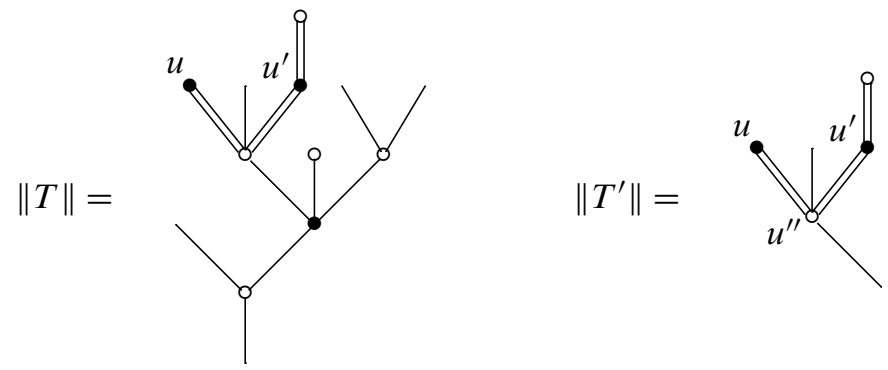

Figure 18: An example of the planted planar tree with leaves $T^{\prime}$ for the relative position of the vertices $u$ and $u^{\prime}$ as in the second diagram of Figure 16. The subcomplex $\operatorname{St}\left(u, u^{\prime}\right) \subset T$ is in double lines.

In this case, by induction hypothesis, the two possible compositions in the square (a) coincide with the morphism (see Figure 19 for an illustration): 


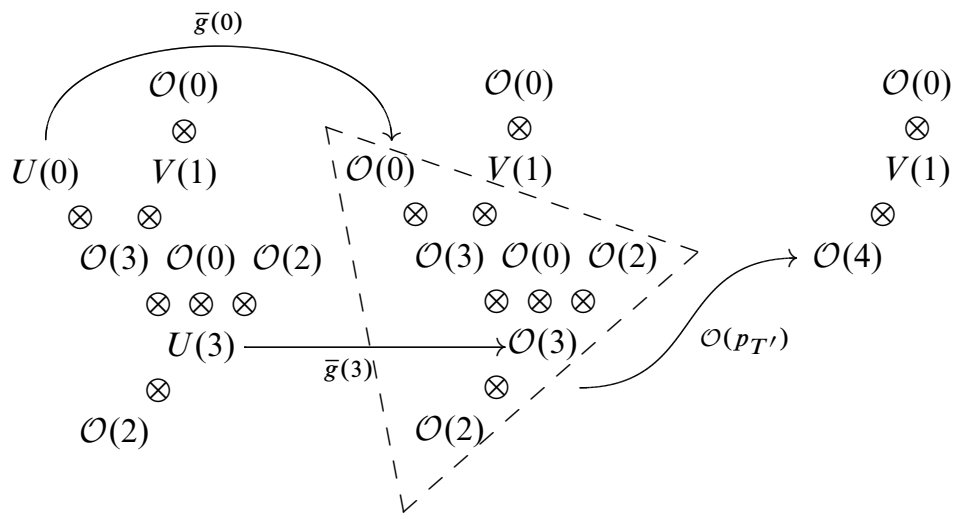

Figure 19: An illustration of Equation (8) for $T, u$ and $u^{\prime}$ as in Figure 17

$$
\begin{aligned}
& U(\tilde{u}) \otimes U\left(\tilde{u^{\prime}}\right) \otimes \otimes V(\widetilde{v}) \otimes \otimes \mathcal{O}(\tilde{w}) \\
& v \in I^{e}(T) \backslash\left\{u, u^{\prime}\right\} \quad w \in I^{o}(T) \\
& \bar{g}(\widetilde{u}) \otimes \bar{g}\left(\tilde{u^{\prime}}\right) \otimes \mathrm{id} \\
& \mathcal{O}(\tilde{u}) \otimes \mathcal{O}\left(\tilde{u^{\prime}}\right) \otimes \otimes V(\widetilde{v}) \otimes \otimes \mathcal{O}(\tilde{w}) \\
& v \in I^{e}(T) \backslash\left\{u, u^{\prime}\right\} \quad w \in I^{o}(T) \\
& \cong \downarrow \text { symmetry } \\
& \otimes V(\tilde{v}) \otimes \otimes \mathcal{O}(\tilde{w}) \otimes \mathcal{O}\left(T^{\prime}\right) \\
& v \in I^{e}(T) \backslash\left\{u, u^{\prime}\right\} \quad w \in I^{o}(T) \backslash \operatorname{Lk}\left(u, u^{\prime}\right) \\
& \downarrow \operatorname{id} \otimes \mathcal{O}\left(p_{T^{\prime}}\right) \\
& \otimes V(\widetilde{v}) \otimes \quad \otimes \mathcal{O}(\widetilde{w}) \otimes \mathcal{O}(m) \\
& v \in I^{e}\left(T / \operatorname{St}\left(u, u^{\prime}\right)\right) \quad w \in I^{o}\left(T / \operatorname{St}\left(u, u^{\prime}\right)\right) \backslash\left\{\left[\operatorname{St}\left(u, u^{\prime}\right)\right]\right\}
\end{aligned}
$$

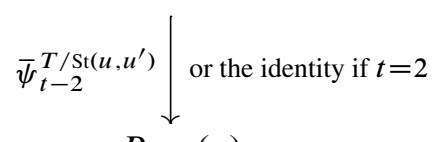

$$
\begin{aligned}
& P_{t-2}(n) \\
& \varphi_{t-1}(n) \downarrow \\
& P_{t-1}(n)
\end{aligned}
$$

(8)

This completes the proof.

In the following lemma, we inductively construct an operad structure on the colimit of the sequence defined in the former. Roughly speaking, we need to define the 
multiplications ${ } i$ of elements attached to $\mathcal{O}$ through planted planar trees with leaves concentrated in even levels $T$ and $T^{\prime}$, where $i$ is less than or equal to the number of leaves of $T$. Consider for instance:
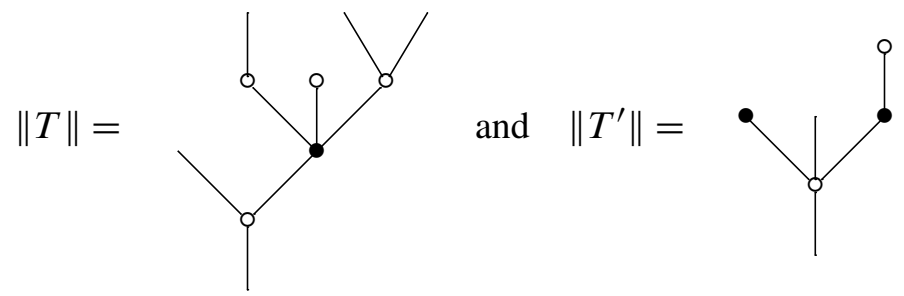

In this case, in order to define $\circ_{2}$ we take $T \circ_{2} T^{\prime}$ and the following associated tensor product of objects in $V$ and $\mathcal{O}$ :

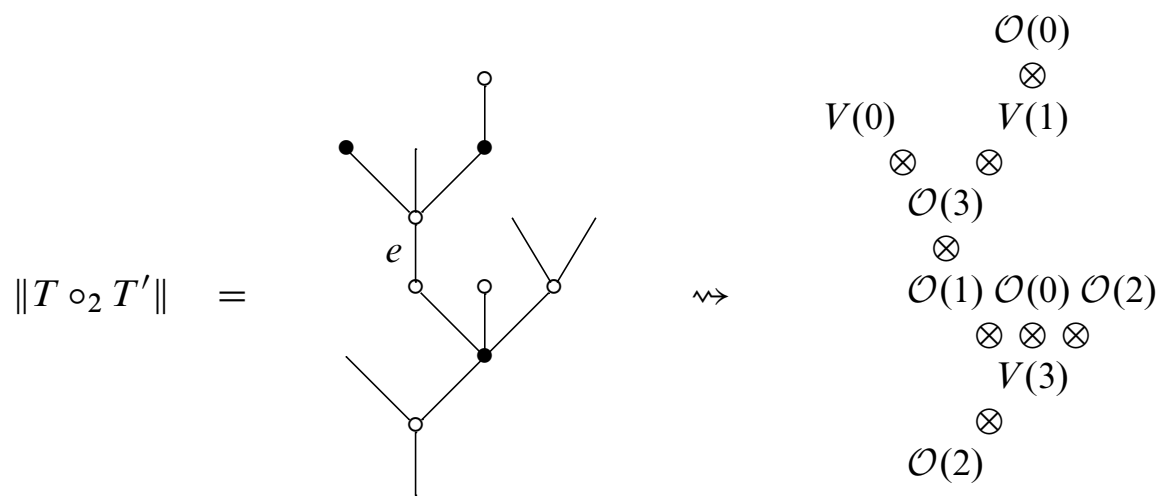

Notice that this object in $\mathscr{V}$ is just the tensor product of the objects associated to $T$ and $T^{\prime}$. Then we contract the root edge $e$ of $T^{\prime}$, which is identified with the second leaf of $T$, and we get a planted planar tree with leaves in even levels $\left(T \circ_{2} T^{\prime}\right) / e$.

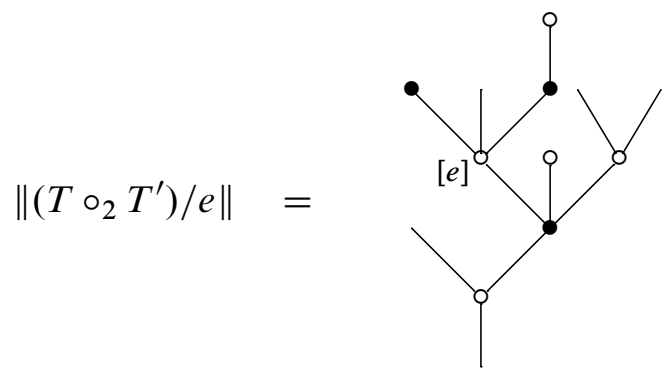

This can be algebraically mimicked on the associated tensor product by means of multiplication in $\mathcal{O}$ according to the local structure of $T \circ_{2} T^{\prime}$ in a neighbourhood 
of $e$, eg $e$ is the second leaf of $T$ but it is the first (and the only) one attached to its inner vertex in $T$ :

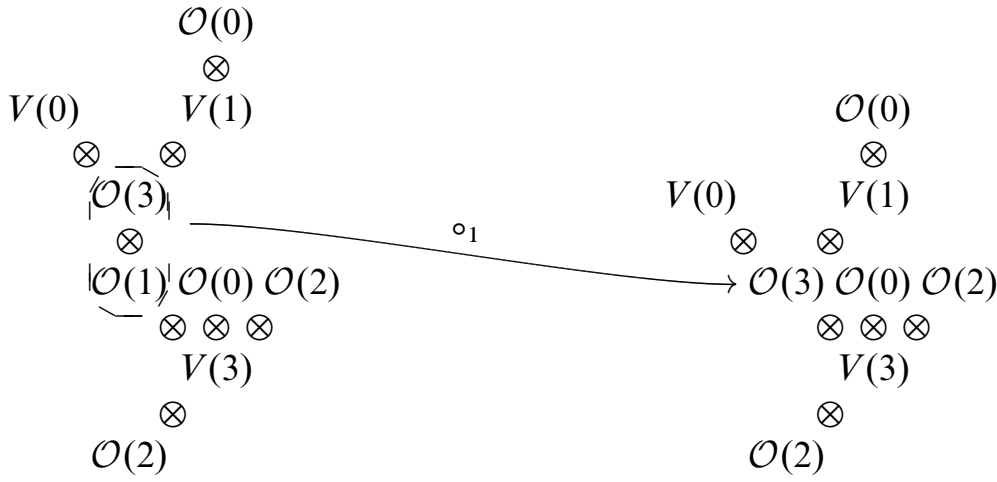

We can define the $\circ_{2}$ multiplication of elements associated to $T$ and $T^{\prime}$ via this morphism and the attaching of elements associated to $\left(T \circ_{2} T^{\prime}\right) / e$. We now formalize this idea.

Lemma 5.2 There are unique morphisms in $\mathscr{V}, n, s, t \geq 0,1 \leq i \leq m$,

$$
c_{i}^{s, t}(m, n): P_{s}(m) \otimes P_{t}(n) \longrightarrow P_{s+t}(m+n-1),
$$

such that

$$
c_{i}^{0,0}=\circ_{i}: \mathcal{O}(m) \otimes \mathcal{O}(n) \longrightarrow \mathcal{O}(m+n-1)
$$

is the operad composition law,

$$
\begin{aligned}
c_{i}^{s, t}(m, n)\left(\varphi_{s}(m) \otimes \mathrm{id}\right) & =\varphi_{s+t}(m+n-1) c_{i}^{s-1, t}(m, n), \\
c_{i}^{s, t}(m, n)\left(\mathrm{id} \otimes \varphi_{t}(n)\right) & =\varphi_{s+t}(m+n-1) c_{i}^{s, t-1}(m, n),
\end{aligned}
$$

and given planted planar trees $T$ and $T^{\prime}$ with leaves concentrated in even levels, card $L(T)=m$, card $L\left(T^{\prime}\right)=n$, card $I^{e}(T)=s$, and card $I^{e}\left(T^{\prime}\right)=t$, if $u^{\prime} \in I^{o}\left(T^{\prime}\right)$ is the unique level 1 vertex, $u \in I^{o}(T)$ belongs to the $i$-th leaf edge (with respect to the path order), the $i$-th leaf edge occupies the $k-t h$ place among all incoming edges of $u$, and $e=\left\{u, u^{\prime}\right\} \in E\left(T \circ_{i} T^{\prime}\right)$, then the morphism $c_{i}^{s, t}(m, n)\left(\bar{\psi}_{s}^{T} \otimes \bar{\psi}_{t}^{T^{\prime}}\right)$ coincides 
with the following morphism that we call $d_{i}^{s, t}\left(T, T^{\prime}\right)$ :

$$
\begin{aligned}
& \bigotimes_{v \in I^{e}(T)} V(\widetilde{v}) \otimes \underset{w \in I^{o}(T)}{\bigotimes} \mathcal{O}(\widetilde{w}) \otimes \underset{v^{\prime} \in I^{e}\left(T^{\prime}\right)}{\bigotimes} V\left(\widetilde{v^{\prime}}\right) \otimes \underset{w^{\prime} \in I^{o}\left(T^{\prime}\right)}{\bigotimes} \mathcal{O}\left(\tilde{w^{\prime}}\right) \\
& \cong \downarrow \text { symmetry }
\end{aligned}
$$

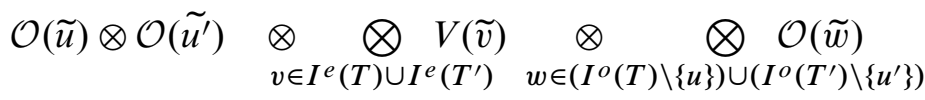

$$
\begin{aligned}
& { }^{\circ} \otimes \mathrm{id} \downarrow
\end{aligned}
$$

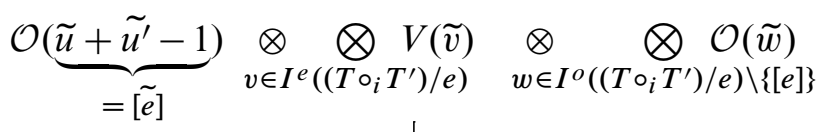

$$
\begin{aligned}
& \bar{\psi}_{s+t}^{\left(T \circ_{i} T^{\prime}\right) / e} \downarrow \\
& P_{s+t}(m+n-1)
\end{aligned}
$$

Here we use the convention that $\bar{\psi}_{0}^{T}=\mathrm{id}_{\mathcal{O}(m)}$ and $\bar{\psi}_{0}^{T^{\prime}}=\mathrm{id}_{\mathcal{O}(n)}$.

Proof The $\operatorname{map} c_{i}^{s, t}(m, n)$ is defined from $c_{i}^{s-1, t}(m, n), c_{i}^{s, t-1}(m, n)$ and $d_{i}^{s, t}\left(T, T^{\prime}\right)$ by using the universal property of the pushout definition of $P_{s}(m) \otimes P_{t}(n)$ arising from Lemma 4.1 and Lemma 5.1 , by induction on $(s, t) \in \mathbb{N} \times \mathbb{N}, \mathbb{N}=\{0,1,2, \ldots\}$, with respect to the graded lexicographic order

$$
(s, t) \leq\left(s^{\prime}, t^{\prime}\right) \Leftrightarrow\left\{\begin{array}{l}
\text { either } s+t<s^{\prime}+t^{\prime}, \\
\text { or } s+t=s^{\prime}+t^{\prime} \text { and } s \leq s^{\prime} .
\end{array}\right.
$$

There is nothing to check for the first three elements $(0,0),(0,1),(1,0)$. Assume that everything holds up to the predecessor of $(s, t)$ with $s+t>1$. We have to show that, for any $x \in I^{e}(T)$ and $x^{\prime} \in I^{e}\left(T^{\prime}\right)$, the following compatibility conditions hold:

(a) $d_{i}^{s, t}\left(T, T^{\prime}\right)(f(\tilde{x}) \otimes \mathrm{id})=\varphi_{s+t}(m+n-1) c_{i}^{s-1, t}(m, n)\left(\psi_{s, x}^{T} \otimes \bar{\psi}_{t}^{T^{\prime}}\right)$.
(b) $d_{i}^{s, t}\left(T, T^{\prime}\right)\left(f\left(\tilde{x^{\prime}}\right) \otimes \mathrm{id}\right)=\varphi_{s+t}(m+n-1) c_{i}^{s, t-1}(m, n)\left(\bar{\psi}_{s}^{T} \otimes \psi_{t, x^{\prime}}^{T^{\prime}}\right)$.

Since (a) and (b) are very similar to each other, we here just check (b). We must distinguish two cases: $\{x, u\} \in E(T)$ and $\{x, u\} \notin E(T)$; see Figure 20. 

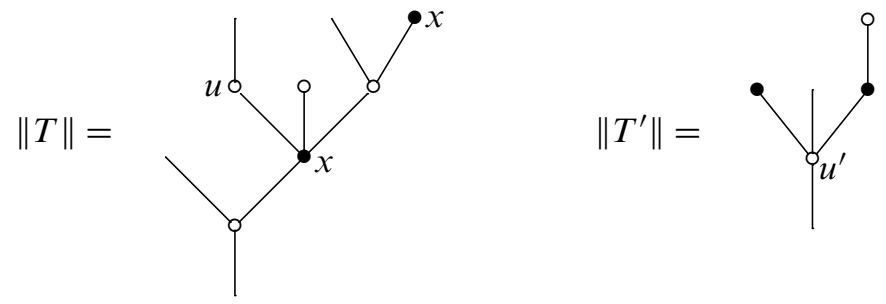

Figure 20: For the trees $T$ and $T^{\prime}$ and $i=2$ we depict $u, u^{\prime}$ and two possible choices of $x$, one with $\{x, u\} \in E(T)$ and the other one with $\{x, u\} \notin E(T)$.

Suppose $\{x, u\} \notin E(T)$. Then $u \notin \operatorname{Lk}(x)$. Using the definition of $d_{i}^{s, t}\left(T, T^{\prime}\right)$ in the statement of this lemma and the definition of $\bar{\psi}_{s+t}^{\left(T \circ_{i} T^{\prime}\right) / e}$ in Lemma 5.1 we deduce that, in this case, the left hand side of (a) is the following composite morphism (see Figure 21):

$$
\begin{aligned}
& U(\tilde{x}) \otimes \otimes V(\widetilde{v}) \otimes \otimes \mathcal{O}(\widetilde{w}) \otimes \otimes V\left(\tilde{v^{\prime}}\right) \otimes \otimes \mathcal{O}\left(\tilde{w^{\prime}}\right) \\
& v \in I^{e}(T) \backslash\{x\} \quad w \in I^{o}(T) \quad v^{\prime} \in I^{e}\left(T^{\prime}\right) \quad w^{\prime} \in I^{o}\left(T^{\prime}\right) \\
& \bar{g}(\tilde{x}) \otimes \mathrm{id} \\
& \mathcal{O}(\tilde{x}) \otimes \otimes V(\widetilde{v}) \otimes \otimes \mathcal{O}(\widetilde{w}) \otimes \otimes V\left(\widetilde{v^{\prime}}\right) \otimes \otimes \mathcal{O}\left(\tilde{w^{\prime}}\right) \\
& v \in I^{e}(T) \backslash\{x\} \quad w \in I^{o}(T) \quad v^{\prime} \in I^{e}\left(T^{\prime}\right) \quad w^{\prime} \in I^{o}\left(T^{\prime}\right) \\
& \cong \downarrow \text { symmetry } \\
& \mathcal{O}(\overline{\operatorname{St}}(x)) \otimes \mathcal{O}(\tilde{u}) \otimes \mathcal{O}\left(\tilde{u^{\prime}}\right)
\end{aligned}
$$

$$
\begin{aligned}
& \otimes \otimes V(\widetilde{v}) \otimes \otimes \mathcal{O}(\tilde{w}) \otimes \otimes V\left(\tilde{v^{\prime}}\right) \otimes \otimes \mathcal{O}\left(\tilde{w^{\prime}}\right) \\
& v \in I^{e}(T) \backslash\{x\} \quad w \in I^{o}(T) \backslash(\operatorname{Lk}(x) \cup\{u\}) \quad v^{\prime} \in I^{e}\left(T^{\prime}\right) \quad w^{\prime} \in I^{o}\left(T^{\prime}\right) \backslash\left\{u^{\prime}\right\} \\
& \begin{aligned}
\mathcal{O}\left(p_{\overline{\mathrm{St}}(x)}\right) \otimes \circ_{k} \otimes \mathrm{id} & \\
\mathcal{O}\left(r_{x}\right) & \otimes \mathcal{O}\left(\tilde{u}+\tilde{u^{\prime}}-1\right)
\end{aligned}
\end{aligned}
$$

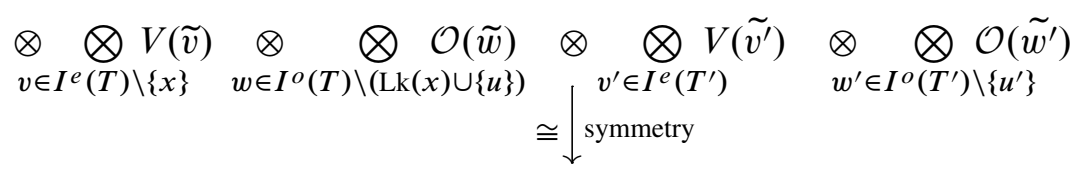$$
\otimes V(\tilde{v}) \quad \otimes \quad \otimes \quad \mathcal{O}(\tilde{w})
$$

$$
\begin{gathered}
v \in I^{e}\left(\left((T / \mathrm{St}(x)) \circ_{i} T^{\prime}\right) / e\right) \\
\bar{\psi}_{s+t-1}^{\left((T / \mathrm{St}(x)) \circ_{i} T^{\prime}\right) / e} \underset{w \in I^{o}\left(\left((T / \mathrm{St}(x)) \circ_{i} T^{\prime}\right) / e\right)}{P_{S+t-1}(m+n-1)} \\
\varphi_{s+t}(m+n-1) \downarrow \\
P_{S+t}(m+n-1)
\end{gathered}
$$


Moreover, by induction, since $(s-1, t)<(s, t)$ one can easily check that this is also the right hand side of (a).
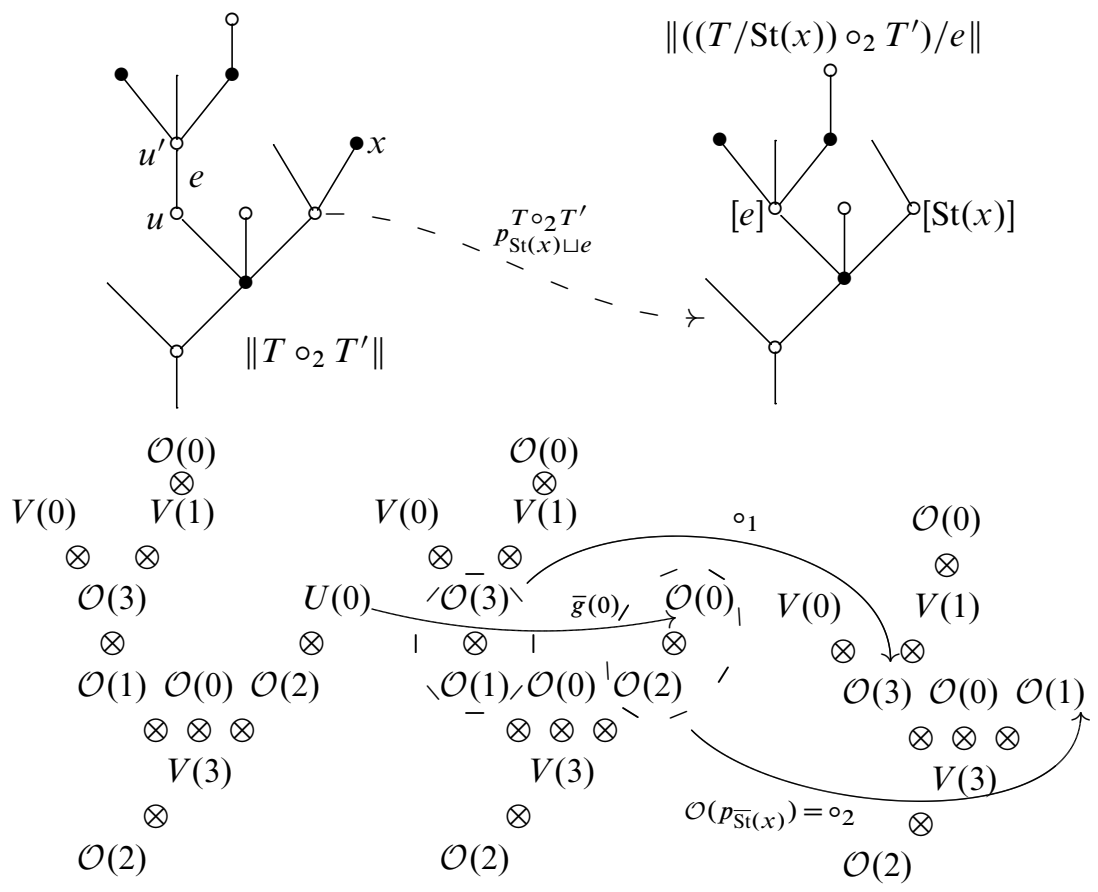

Figure 21: An illustration of Equation (9) for $T$ and $T^{\prime}$ as in Figure 20 in case $\{x, u\} \notin E(T)$

Suppose now that $\{x, u\} \in E(T)$. Then $u \in \operatorname{Lk}(x)$. Assume that $e$ is the $l$-th leaf of $\overline{\operatorname{St}}(x)$. We denote $T^{\prime \prime}$ the planted planar tree with leaves $T^{\prime \prime}=\overline{\operatorname{St}}(x){ }_{l} C_{\tilde{u}^{\prime}}$. The inner part of $T^{\prime \prime}$ is identified with the subtree $T^{\prime \prime \prime} \subset T \circ_{i} T^{\prime}$ formed by adjoining the edge $e$ to $\operatorname{St}(x)$; see Figure 22. Using the definition of $d_{i}^{s, t}\left(T, T^{\prime}\right)$ in the statement,
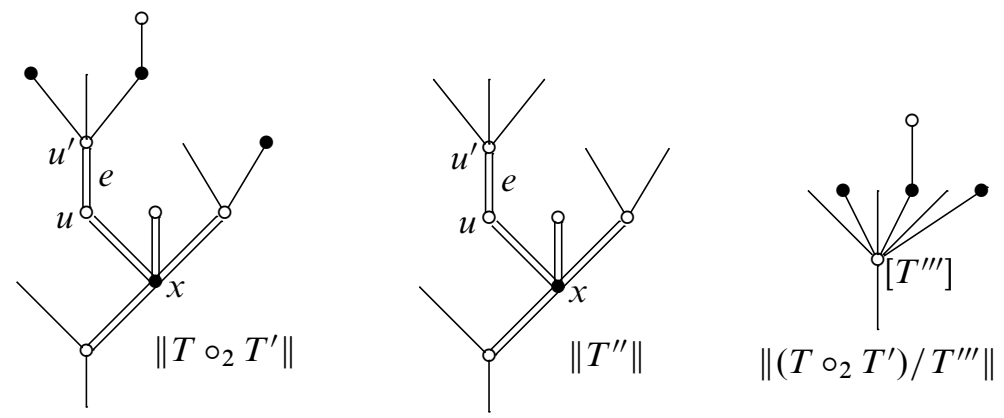

Figure 22: For the choice of $x$ in Figure 20 with $\{x, u\} \in E(T)$ we here depict $T^{\prime \prime}$. The subtree $T^{\prime \prime \prime}$ is indicated with double lines. 
the definition of $\bar{\psi}_{s+t}^{\left(T \circ_{i} T^{\prime}\right) / e}$ in Lemma 5.1, and relation (2) in Remark 2.6 for $\mathcal{O}$, we deduce that, in this case, the left hand side of (a) is the following composite morphism (see Figure 23):

(10)

$$
\begin{aligned}
& U(\tilde{x}) \otimes \otimes V(\widetilde{v}) \otimes \otimes \mathcal{O}(\widetilde{w}) \otimes \otimes V\left(\widetilde{v^{\prime}}\right) \otimes \otimes \mathcal{O}\left(\tilde{w^{\prime}}\right) \\
& v \in I^{e}(T) \backslash\{x\} \quad w \in I^{o}(T) \quad v^{\prime} \in I^{e}\left(T^{\prime}\right) \quad w^{\prime} \in I^{o}\left(T^{\prime}\right) \\
& \bar{g}(\widetilde{x}) \otimes \text { id } \\
& \mathcal{O}(\tilde{x}) \otimes \otimes V(\widetilde{v}) \otimes \otimes \mathcal{O}(\widetilde{w}) \otimes \otimes V\left(\widetilde{v^{\prime}}\right) \otimes \otimes \mathcal{O}\left(\tilde{w^{\prime}}\right) \\
& v \in I^{e}(T) \backslash\{x\} \quad w \in I^{o}(T) \quad v^{\prime} \in I^{e}\left(T^{\prime}\right) \quad w^{\prime} \in I^{o}\left(T^{\prime}\right) \\
& \cong \downarrow \text { symmetry }
\end{aligned}
$$

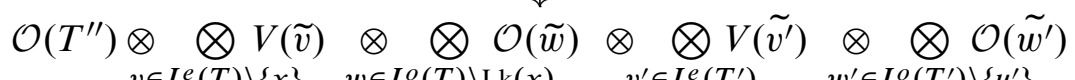

$$
\begin{aligned}
& v \in I^{e}(T) \backslash\{x\} \quad w \in I^{o}(T) \backslash \operatorname{Lk}(x) \quad v^{\prime} \in I^{e}\left(T^{\prime}\right) \quad w^{\prime} \in I^{o}\left(T^{\prime}\right) \backslash\left\{u^{\prime}\right\} \\
& \mathcal{O}\left(p_{T^{\prime \prime}}\right) \otimes \mathrm{id} \\
& \mathcal{O}\left(r_{x}+\tilde{u^{\prime}}-1\right) \otimes \otimes V(\widetilde{v}) \otimes \otimes \mathcal{O}(\tilde{w}) \otimes \otimes V\left(\widetilde{v^{\prime}}\right) \otimes \otimes \mathcal{O}\left(\tilde{w^{\prime}}\right) \\
& v \in I^{e}(T) \backslash\{x\} \quad w \in I^{o}(T) \backslash \operatorname{Lk}(x) \quad v^{\prime} \in I^{e}\left(T^{\prime}\right) \quad w^{\prime} \in I^{o}\left(T^{\prime}\right) \backslash\left\{u^{\prime}\right\} \\
& \cong \downarrow \text { symmetry } \\
& \otimes V(\tilde{v}) \quad \otimes \quad \otimes \mathcal{O}(\tilde{w}) \\
& v \in I^{e}\left(\left(T \circ_{i} T^{\prime}\right) / T^{\prime \prime \prime}\right) \quad w \in I^{o}\left(\left(T \circ_{i} T^{\prime}\right) / T^{\prime \prime \prime}\right) \\
& \bar{\psi}_{s+t-1}^{\left(T \circ_{i} T^{\prime}\right) / T^{\prime \prime \prime}} \downarrow \\
& P_{s+t-1}(m+n-1) \\
& \varphi_{s+t}(m+n-1) \downarrow \\
& P_{s+t}(m+n-1)
\end{aligned}
$$

Moreover, by induction one can easily check that this is also the right hand side of (a), hence we are done with this proof.

Let $\mathcal{P}$ be the sequence defined as

$$
\mathcal{P}(n)=\operatorname{colim}_{t \geq 0} P_{t}(n) .
$$

By the previous lemma, the morphisms $c_{i}^{s, t}(m, n)$ induce composition laws in the colimit:

$$
\circ_{i}: \mathcal{P}(m) \otimes \mathcal{P}(n) \longrightarrow \mathcal{P}(m+n-1), \quad 1 \leq i \leq m, n \geq 0 .
$$

Consider the morphism

$$
\mathbb{I} \stackrel{u}{\longrightarrow} \mathcal{O}(1)=P_{0}(1) \stackrel{\text { canonical }}{\longrightarrow} \underset{t \geq 0}{\operatorname{colim}} P_{t}(1)=\mathcal{P}(1) .
$$




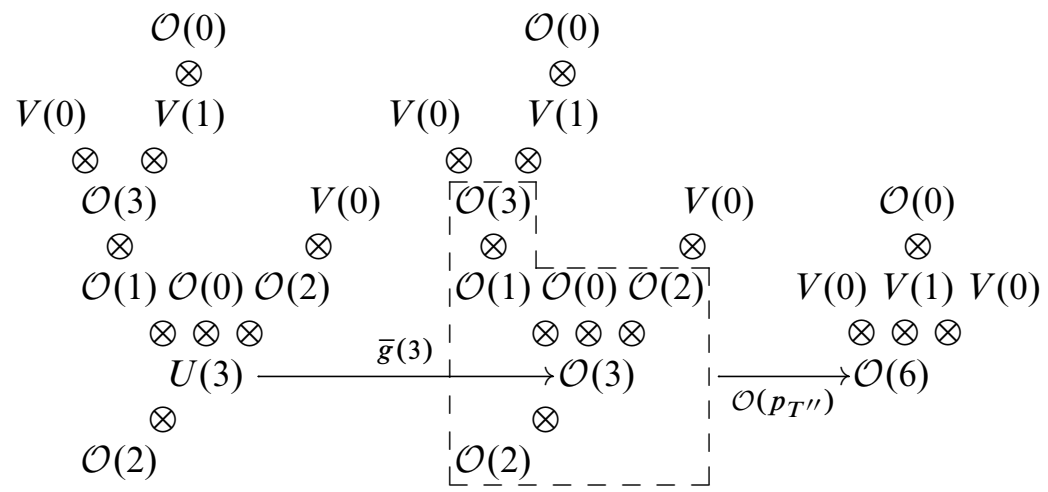

Figure 23: An illustration of Equation (10) for $T$ and $T^{\prime}$ as in Figure 20 in case $\{x, u\} \in E(T)$ (see Figure 22)

Proposition 5.3 The sequence $\mathcal{P}$, the unit in Equation (12) and the composition laws in Equation (11) define an operad.

Proof We must check that relations (1)-(4) in Remark 2.6 hold for $\mathcal{P}$. Each of these relations for $\mathcal{P}$ can be derived from the corresponding relation for $\mathcal{O}$. As relations (1) and (2) are very similar to each other, just as (3) and (4), we here check (2) and (3).

In order to prove relation (2) for $\mathcal{P}$ it is enough to check that the following two morphisms $P_{r}(l) \otimes P_{s}(m) \otimes P_{t}(n) \rightarrow P_{r+s+t}(l+m+n-2)$ coincide:

$c_{j}^{r+s, t}(l+m-1, n)\left(c_{i}^{r, s}(l, m) \otimes \operatorname{id}_{P_{t}(n)}\right)=c_{i}^{r, s+t}(l, m+n-1)\left(\operatorname{id}_{P_{r}(l)} \otimes c_{j-i+1}^{s, t}(m, n)\right)$.

We check this by induction on $(r, s, t) \in \mathbb{N}^{3}$ with respect to the graded lexicographic order. For $r=s=t=0$ this is just relation (2) for the operad $\mathcal{O}$. If we assume that the relation holds up to the predecessor of $(r, s, t)$, then by using the universal property of the pushout definition of $P_{r}(m) \otimes P_{s}(n) \otimes P_{t}(p)$ arising from Lemmas 4.1 and 5.1, we only have to check that, with the notation of Lemma 5.2, given planted planar trees with leaves concentrated in even levels $T, T^{\prime}, T^{\prime \prime}$ with $\operatorname{card} L(T)=l, \operatorname{card} L\left(T^{\prime}\right)=m$, $\operatorname{card} L\left(T^{\prime \prime}\right)=n, \operatorname{card} I^{e}(T)=r, \operatorname{card} I^{e}\left(T^{\prime}\right)=s$, and $\operatorname{card} I^{e}\left(T^{\prime \prime}\right)=t$, then

$$
\begin{aligned}
c_{j}^{r+s, t}(l+m-1, n)\left(d_{i}^{r, s}\left(T, T^{\prime}\right)\right. & \left.\otimes \bar{\psi}_{t}^{T^{\prime \prime}}\right) \\
& =c_{i}^{r, s+t}(l, m+n-1)\left(\bar{\psi}_{r}^{T} \otimes d_{j-i+1}^{s, t}\left(T^{\prime}, T^{\prime \prime}\right)\right) .
\end{aligned}
$$

Let $u \in I^{o}(T)$ be the inner vertex of the $i$-th leaf edge of $T, u_{1}^{\prime} \in I^{o}\left(T^{\prime}\right)$ the unique level 1 vertex of $T^{\prime}, u_{2}^{\prime} \in I^{o}\left(T^{\prime}\right)$ the inner vertex of the $(j-i+1)$-st leaf edge of $T^{\prime}$, and $u^{\prime \prime} \in I^{o}\left(T^{\prime \prime}\right)$ the unique level 1 vertex of $T^{\prime \prime}$. Suppose that the $i$-th leaf edge 
of $T$ is the $k_{1}$-st incoming edge of $u$, and that the $(j-i+1)$-st leaf edge of $T^{\prime}$ is the $k_{2}$-th incoming edge of $u_{2}^{\prime}$. The most complicated case is when $u_{1}^{\prime}=u_{2}^{\prime}$, and even this case is easy, although somewhat tedious.
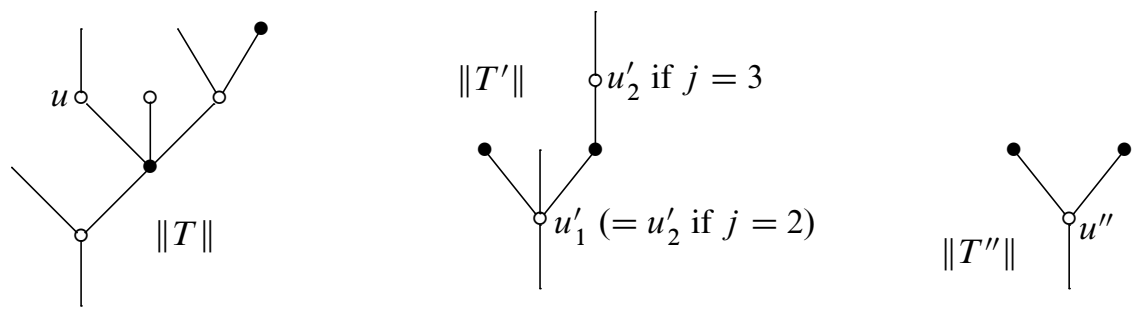

Figure 24: For the planted planar trees with leaves $T, T^{\prime}$ and $T^{\prime \prime}$ we depict $u, u_{1}^{\prime}, u_{2}^{\prime}$ and $u^{\prime \prime}$ for $i=2$ and $j=2,3$.

Assume $u_{1}^{\prime}=u_{2}^{\prime}$ and denote this vertex simply by $u^{\prime}$. Notice that $\left(T \circ_{i} T^{\prime}\right) \circ_{j} T^{\prime \prime}=$ $T \circ_{i}\left(T^{\prime} \circ_{j-i+1} T^{\prime \prime}\right)$; compare the second tree in Figure 10. Let $K \subset\left(T \circ_{i} T^{\prime}\right) \circ_{j} T^{\prime \prime}$ be the subtree with $V(K)=\left\{u, u^{\prime}, u^{\prime \prime}\right\}$ and $E(K)=\left\{\left\{u, u^{\prime}\right\},\left\{u^{\prime}, u^{\prime \prime}\right\}\right\}$; see Figure 25 . Then by Lemma 5.2 and relation (2) for $\mathcal{O}$, both sides of (a) coincide with the following:

$$
\begin{aligned}
& \otimes V(\widetilde{v}) \otimes \otimes \mathcal{O}(\tilde{w}) \otimes \otimes V\left(\tilde{v^{\prime}}\right) \otimes \otimes \mathcal{O}\left(\tilde{w^{\prime}}\right) \otimes \otimes V\left(\tilde{v^{\prime \prime}}\right) \otimes \otimes \mathcal{O}\left(\tilde{w^{\prime \prime}}\right) \\
& v \in I^{e}(T) \quad w \in I^{o}(T) \quad v^{\prime} \in I^{e}\left(T^{\prime}\right) \quad w^{\prime} \in I^{o}\left(T^{\prime}\right) \quad v^{\prime \prime} \in I^{e}\left(T^{\prime \prime}\right) \quad w^{\prime \prime} \in I^{o}\left(T^{\prime \prime}\right) \\
& \cong \downarrow \text { symmetry } \\
& \mathcal{O}(\tilde{u}) \otimes \mathcal{O}\left(\tilde{u^{\prime}}\right) \otimes \mathcal{O}\left(\tilde{u^{\prime \prime}}\right) \quad \otimes_{v \in I^{e}(T) \cup I^{e}\left(T^{\prime}\right) \cup I^{e}\left(T^{\prime \prime}\right)} V(\tilde{w}) \otimes_{w \in\left(I^{o}(T) \cup I^{o}\left(T^{\prime}\right) \cup I^{o}\left(T^{\prime \prime}\right)\right) \backslash\left\{u, u^{\prime}, u^{\prime \prime}\right\}}^{\otimes} \\
& \left(\circ_{k_{1}}\left(\mathrm{id} \otimes \circ_{k_{2}}\right)\right) \otimes \mathrm{id}
\end{aligned}
$$

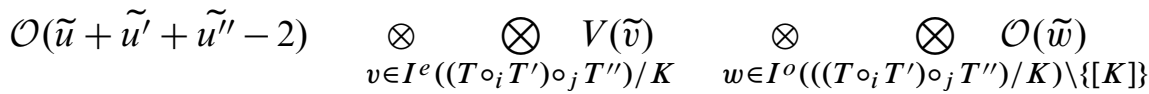

$$
\begin{aligned}
& \bar{\psi}_{r+s+t}^{\left(\left(T \circ_{i} T^{\prime}\right) \circ_{j} T^{\prime \prime}\right) / K} \downarrow \\
& P_{s+t}(m+n+p-2)
\end{aligned}
$$

Assume now that $u_{1}^{\prime} \neq u_{2}^{\prime}$. In this case it is not even necessary to use any of the relations in Remark 2.6 for $\mathcal{O}$. Actually, by Lemma 5.2, if $K \subset\left(T \circ_{i} T^{\prime}\right) \circ_{j} T^{\prime \prime}$ is the (disjoint) union of the edges $e_{1}=\left\{u, u_{1}^{\prime}\right\}$ and $e_{2}=\left\{u_{2}^{\prime}, u^{\prime \prime}\right\}$; see Figure 25, then both 

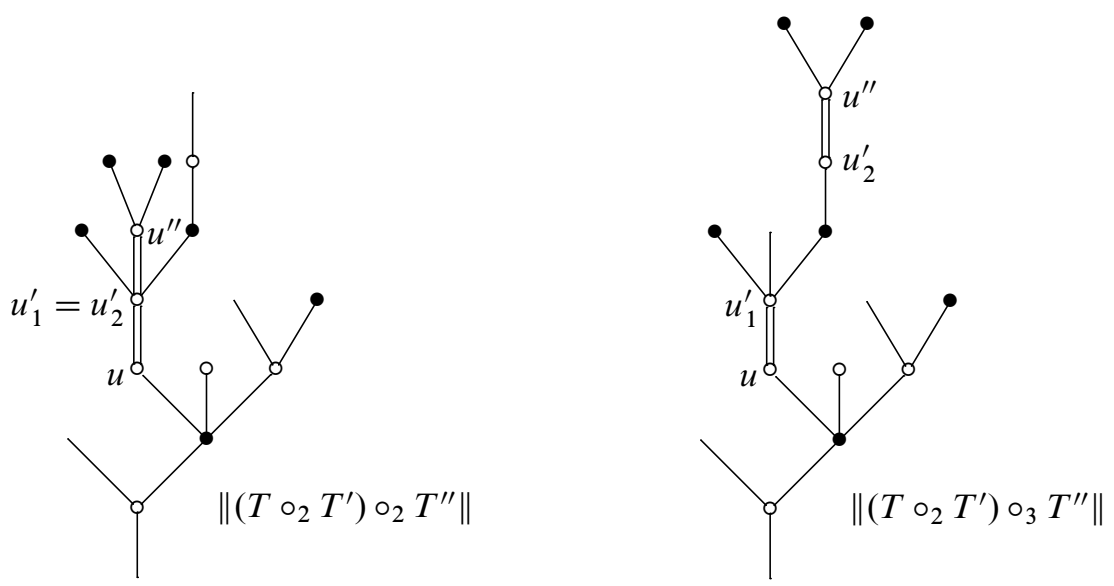

Figure 25: Here we depict the subtree $K \subset\left(T \circ_{i} T^{\prime}\right) \circ_{j} T^{\prime \prime}$ in double lines for the trees in Figure 24, $i=2$ and $j=2,3$.

sides of (a) coincide with the following:

$$
\begin{aligned}
& \otimes V(\widetilde{v}) \otimes \otimes \mathcal{O}(\tilde{w}) \otimes \otimes V\left(\tilde{v^{\prime}}\right) \otimes \otimes \mathcal{O}\left(\tilde{w^{\prime}}\right) \otimes \otimes V\left(\tilde{v^{\prime \prime}}\right) \otimes \otimes \mathcal{O}\left(\tilde{w^{\prime \prime}}\right) \\
& v \in I^{e}(T) \quad w \in I^{o}(T) \quad v^{\prime} \in I^{e}\left(T^{\prime}\right) \quad w^{\prime} \in I^{o}\left(T^{\prime}\right) \quad v^{\prime \prime} \in I^{e}\left(T^{\prime \prime}\right) \quad w^{\prime \prime} \in I^{o}\left(T^{\prime \prime}\right) \\
& \cong \downarrow \text { symmetry }
\end{aligned}
$$

$\mathcal{O}(\widetilde{u}) \otimes \mathcal{O}\left(\tilde{u_{1}^{\prime}}\right) \otimes \mathcal{O}\left(\tilde{u_{2}^{\prime}}\right) \otimes \mathcal{O}\left(\tilde{u^{\prime \prime}}\right) \otimes \otimes \quad V(\widetilde{v}) \quad \otimes \quad \otimes \quad \mathcal{O}(\widetilde{w})$ $v \in I^{e}(T) \cup I^{e}\left(T^{\prime}\right) \cup I^{e}\left(T^{\prime \prime}\right) w \in\left(I^{o}(T) \cup I^{o}\left(T^{\prime}\right) \cup I^{o}\left(T^{\prime \prime}\right)\right) \backslash\left\{u, u_{1}^{\prime}, u_{2}^{\prime}, u^{\prime \prime}\right\}$

$$
\begin{aligned}
& \circ_{k_{1}} \otimes \circ_{k_{2}} \otimes \mathrm{id} \downarrow \\
& \mathcal{O}\left(\tilde{u}+\tilde{u_{1}^{\prime}}-1\right) \otimes \mathcal{O}\left(\tilde{u_{2}^{\prime}}+\tilde{u^{\prime \prime}}-1\right) \otimes \bigotimes_{v \in I^{e}\left(\left(T \circ_{i} T^{\prime}\right) \circ_{j} T^{\prime \prime}\right) / K} V(\widetilde{v}) \underset{w \in I^{o}\left(\left(\left(T \circ_{i} T^{\prime}\right) \circ_{j} T^{\prime \prime}\right) / K\right) \backslash\left\{\left[e_{1}\right],\left[e_{2}\right]\right\}}{\otimes} \underset{\mathcal{O}(\tilde{w})}{\otimes}
\end{aligned}
$$

$$
\begin{gathered}
\bar{\psi}_{r+s+t}^{\left(\left(T \circ_{i} T^{\prime}\right) \circ_{j} T^{\prime \prime}\right) / K} \downarrow \\
P_{s+t}(m+n+p-2)
\end{gathered}
$$

Relation (3) is a consequence of the fact that the following composite morphism is a right unit constraint in $\mathscr{V}$ :

$$
P_{r}(l) \otimes \mathbb{I} \stackrel{\mathrm{id} \otimes u}{\longrightarrow} P_{r}(l) \otimes \mathcal{O}(1)=P_{r}(l) \otimes P_{0}(1) \stackrel{c_{i}^{r, 0}(l, 1)}{\longrightarrow} P_{r}(l) .
$$

This follows by induction on $r$. For $r=0$ this is just relation (3) for $\mathcal{O}$. Assume this holds up to $r-1$. By Lemma 5.1 and the induction hypothesis, we only have to check that the morphism $c_{i}^{r, 0}(l, 1)\left(\bar{\psi}_{r}^{T} \otimes u\right)$ coincides with the composition of the right unit isomorphism and $\bar{\psi}_{r}^{T}$. By Lemma 5.2,

$$
c_{i}^{r, 0}(l, 1)\left(\bar{\psi}_{r}^{T} \otimes \operatorname{id}_{\mathcal{O}(1)}\right)=d_{i}^{r, 0}\left(T, C_{1}\right) .
$$


Let $u^{\prime} \in I^{o}\left(C_{1}\right)$ be now the unique inner vertex of $C_{1}$, and $e=\left\{u, u^{\prime}\right\} \in E\left(T \circ_{i} C_{1}\right)$. In this case $\left(T \circ_{i} C_{1}\right) / e=T$. Moreover, by the definition of $d_{i}^{r, 0}\left(T, C_{1}\right)$ in the statement of Lemma 5.2 and by relation (3) for $\mathcal{O}$, the morphism $d_{i}^{r, 0}\left(T, C_{1}\right)(\mathrm{id} \otimes u)$ is the composition of the right unit isomorphism and $\bar{\psi}_{r}^{T}$, hence we are done.

Consider the morphisms of sequences $f^{\prime}: \mathcal{O} \rightarrow \mathcal{P}$ and $\bar{g}^{\prime}: V \rightarrow \mathcal{P}$ defined as:

$$
f^{\prime}(n): \mathcal{O}(n)=P_{0}(n) \stackrel{\text { canonical }}{\longrightarrow} \underset{t \geq 0}{\operatorname{colim}} P_{t}(n)=\mathcal{P}(n),
$$
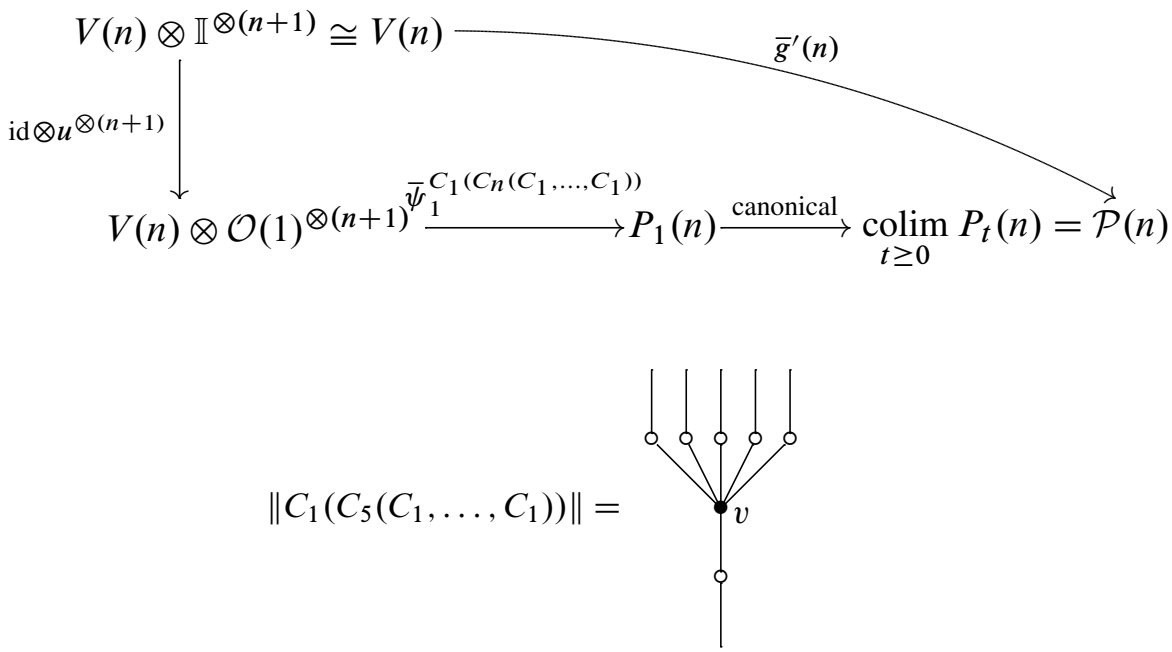

Figure 26: The planted planar tree with leaves in even levels $C_{1}\left(C_{n}\left(C_{1}, \ldots, C_{1}\right)\right)$ for $n=5$

Theorem 5.4 The morphism $f^{\prime}: \mathcal{O} \rightarrow \mathcal{P}$ is an operad morphism. Moreover, if $g^{\prime}: \mathcal{F}(V) \rightarrow \mathcal{P}$ is the operad morphism adjoint to $\bar{g}^{\prime}$, then the following diagram is a pushout in $\mathrm{Op}(\mathscr{V})$ :

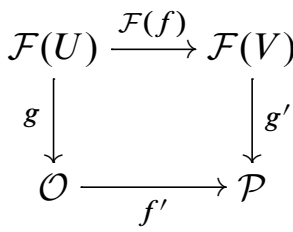

Proof The morphism $f^{\prime}$ is an operad morphism by the very definition of the operad structure in $\mathcal{P}$, since $c_{i}^{0,0}=o_{i}$ is the structure morphism of $\mathcal{O}$ and the unit of $\mathcal{P}$ is the composition of the unit of $\mathcal{O}$ and $f^{\prime}$; see Lemma 5.2 and Equation (12). Moreover, 
the square

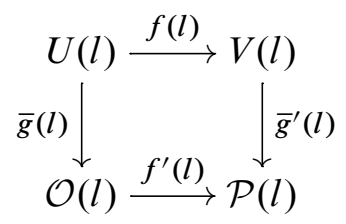

commutes for all $l \geq 0$. In fact, the following diagram commutes by some trivial facts, including the very definition of $P_{1}(l)$ in Lemma 5.1:

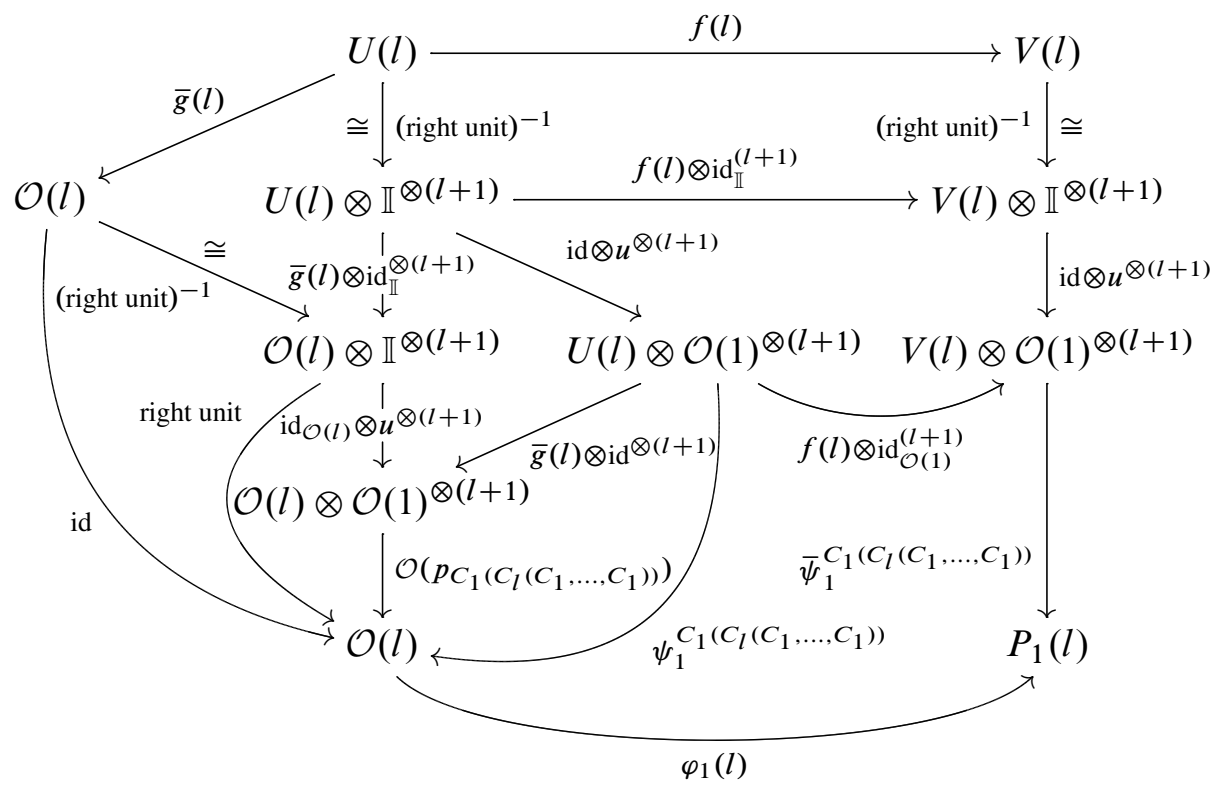

Also, its outer (commutative) square

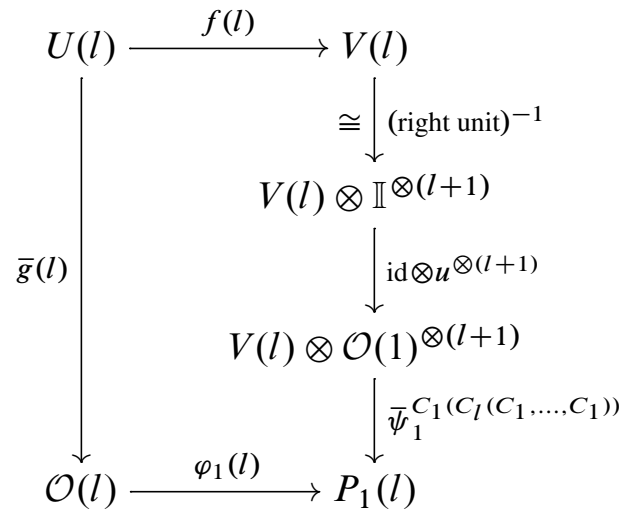

composed with the canonical morphism $P_{1}(l) \rightarrow \operatorname{colim}_{r \geq 0} P_{r}(l)=\mathcal{P}(l)$ yields the former square. 
Suppose we are given an operad $\mathcal{P}^{\prime}$ and morphisms $f^{\prime \prime}: \mathcal{O} \rightarrow \mathcal{P}^{\prime}$ in $\operatorname{Op}(\mathscr{V})$ and $\bar{g}^{\prime \prime}: V \rightarrow \mathcal{P}^{\prime}$ in $\mathscr{V}^{\mathbb{N}}$ such that the square

(a)

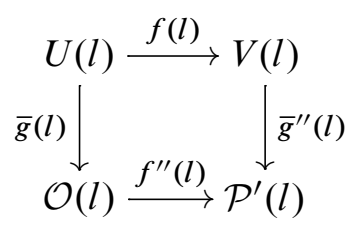

commutes for all $l \geq 0$. We must show that there is a unique morphism $h: \mathcal{P} \rightarrow \mathcal{P}^{\prime}$ in Op $(\mathscr{V})$ such that $f^{\prime \prime}=h f^{\prime}$ and $\bar{g}^{\prime \prime}=h \bar{g}^{\prime}$ in $\mathscr{V}^{\mathbb{N}}$.

We define morphisms

$$
h_{r}(l): P_{r}(l) \longrightarrow \mathcal{P}^{\prime}
$$

by induction on $r \geq 0$ as follows. We set $h_{0}(l)=f^{\prime \prime}(l)$. Assume we have defined up to $h_{r-1}(l)$. Then we define $h_{r}(l)$ so that $h_{r}(l) \varphi_{r}(l)=h_{r-1}(l)$ and, for any planted planar tree $T$ with $l$ leaves concentrated in even levels and $r$ inner vertices in even levels:

$$
h_{r}(l) \bar{\psi}_{r}^{T}=\mathcal{P}^{\prime}\left(p_{T}\right)\left(\bigotimes_{v \in I^{e}(T)} \bar{g}^{\prime \prime}(\widetilde{v}) \otimes \bigotimes_{w \in I^{o}(T)} f^{\prime \prime}(\widetilde{w})\right)
$$

The morphism $h_{r}(l)$ is well defined by the universal property of the pushout definition of $P_{r}(l)$ in Lemma 5.1 since, given $u \in I^{e}(T)$,

$$
\begin{aligned}
& \mathcal{P}^{\prime}\left(p_{T}\right)\left(\bigotimes_{v \in I^{e}(T)} \bar{g}^{\prime \prime}(\widetilde{v}) \otimes \bigotimes_{w \in I^{o}(T)} f^{\prime \prime}(\widetilde{w})\right)(f(\widetilde{u}) \otimes \mathrm{id}) \\
& =\mathcal{P}^{\prime}\left(p_{T}\right)\left(\bigotimes_{v \in I^{e}(T) \backslash\{u\}} \bar{g}^{\prime \prime}(\widetilde{v}) \otimes \bigotimes_{w \in I^{o}(T) \cup\{u\}} f^{\prime \prime}(\widetilde{w})\right)(\mathrm{id} \otimes \bar{g}(\widetilde{u})) \\
& =\mathcal{P}^{\prime}\left(p_{T / \operatorname{St}(u)}\right)(\bigotimes_{v \in I^{e}(T / \operatorname{St}(u)) \quad \bar{g}^{\prime \prime}(\widetilde{v}) \otimes I^{o}(T / \operatorname{St}(u))} \underbrace{\prime \prime}(\tilde{w}))\left(\mathrm{id} \otimes \mathcal{O}\left(p_{\overline{\operatorname{St}}(u)}\right)\right)(\mathrm{id} \otimes \bar{g}(\tilde{u})) \\
& =h_{r-1}(l) \bar{\psi}_{r-1}^{T / \operatorname{St}(u)}\left(\operatorname{id} \otimes \mathcal{O}\left(p_{\overline{\operatorname{St}}(u)}\right)\right)(\mathrm{id} \otimes \bar{g}(\tilde{u})) \\
& =h_{r-1}(l) \psi_{r, u}^{T} .
\end{aligned}
$$

Here, in the first equation we use the commutativity of (a), in the second equation we use the fact that $f^{\prime \prime}$ is an operad morphism, and in the third equation we use the induction hypothesis. The fourth equation follows from the very definition of $\psi_{r, u}^{T}$ in the statement of Lemma 5.1. For simplicity, in these equations we have omitted some symmetry isomorphisms in $\mathscr{V}$. 
We have checked that the morphisms $h_{r}(l)$ induce a morphism of sequences $h: \mathcal{P} \rightarrow \mathcal{P}^{\prime}$ in the colimit. It is clear that $h f^{\prime}=f^{\prime \prime}$ since $h_{0}=f^{\prime \prime}$, in particular $h$ preserves units. Moreover, $h g^{\prime}=g^{\prime \prime}$ since for $\bar{T}=C_{1}\left(C_{l}\left(C_{1}, \ldots, C_{1}\right)\right.$ ) (see Figure 26), if $v \in I^{e}(\bar{T})$ is the unique inner vertex in even levels, then

$$
\begin{aligned}
h_{1}(l) \bar{\psi}_{1}^{\bar{T}}\left(\operatorname{id}_{V(l)} \otimes u^{\otimes(l+1)}\right) & =\mathcal{P}^{\prime}\left(p_{\bar{T}}\right)\left(\bar{g}^{\prime \prime}(l) \otimes f^{\prime \prime}(1)^{\otimes(l+1)}\right)\left(\operatorname{id}_{V(l)} \otimes u^{\otimes(l+1)}\right) \\
& =\mathcal{P}^{\prime}\left(p_{\bar{T}}\right)\left(\bar{g}^{\prime \prime}(l) \otimes u^{\otimes(l+1)}\right) \\
& =\bar{g}^{\prime \prime}(l) .
\end{aligned}
$$

Here, in the first equation we use (b), in the second equation we use that $f^{\prime \prime}$ is an operad morphism and therefore it preserves units, and in the third equation we use relations (3) and (4) in Remark 2.6 for the operad $\mathcal{P}^{\prime}$.

In order to check that $h$ is indeed an operad morphism, we show that

$$
h_{r+s}(l+m-1) c_{i}^{r, s}(l, m)=h_{r}(l) \circ_{i} h_{s}(m) .
$$

We proceed by induction on $(r, s) \in \mathbb{N}^{2}$ with respect to the graded lexicographic order. This is obvious for $r=s=0$, since $f^{\prime \prime}$ is an operad morphism. If the equation holds up to the predecessor of $(r, s)$ then by induction hypothesis we only have to check that the following equation holds:

$$
h_{r+s}(l+m-1) c_{i}^{r, s}(l, m)\left(\bar{\psi}_{r}^{T} \otimes \bar{\psi}_{s}^{T^{\prime}}\right)=\left(h_{r}(l) \bar{\psi}_{r}^{T}\right) \circ_{i}\left(h_{s}(m) \bar{\psi}_{s}^{T^{\prime}}\right),
$$

for $T^{\prime}$ a planted planar tree with $m$ leaves concentrated in even levels and $s$ inner vertices in even levels. Let $u \in I^{o}(T)$ be the inner vertex of the $i$-th leaf edge of $T$, $u^{\prime} \in I^{o}\left(T^{\prime}\right)$ the unique level 1 vertex of $T^{\prime}$, and $e=\left\{u, u^{\prime}\right\} \in E\left(T \circ_{i} T^{\prime}\right)$. Suppose that the $i$-th leaf edge of $T$ is the $k$-th incoming edge of $u$. Then,

$$
\begin{aligned}
& h_{r+s}(l+m-1) c_{i}^{r, s}(l, m)\left(\bar{\psi}_{r}^{T} \otimes \bar{\psi}_{s}^{T^{\prime}}\right) \\
& =h_{r+s}(l+m-1) d_{i}^{r, s}\left(T, T^{\prime}\right) \\
& =h_{r+s}(l+m-1) \bar{\psi}_{s+t}^{\left(T \circ_{i} T^{\prime}\right) / e}\left(\circ_{k} \otimes \mathrm{id}\right) \\
& =\mathcal{P}^{\prime}\left(p_{\left(T \circ_{i} T^{\prime}\right) / e}\right)\left(\bigotimes_{v \in I^{e}\left(\left(T \circ_{i} T^{\prime}\right) / e\right)} \bar{g}^{\prime \prime}(\widetilde{v}) \otimes \bigotimes_{w \in I^{o}\left(\left(T \circ_{i} T^{\prime}\right) / e\right)} f^{\prime \prime}(\tilde{w})\right)\left(\circ_{k} \otimes \mathrm{id}\right) \\
& =\mathcal{P}^{\prime}\left(p_{\left(T \circ_{i} T^{\prime}\right) / e}\right)\left(\circ_{k} \otimes \mathrm{id}\right)\left(\begin{array}{c}
\bigotimes_{v \in I^{e}(T) \cup I^{e}\left(T^{\prime}\right) \quad}^{\bar{g}^{\prime \prime}(\tilde{v}) \otimes I^{o}(T) \cup I^{o}\left(T^{\prime}\right)}
\end{array} \bigotimes_{w}^{f^{\prime \prime}(\tilde{w})}\right)
\end{aligned}
$$




$$
\begin{aligned}
& =\left(\mathcal{P}^{\prime}\left(p_{T}\right) \circ_{i} \mathcal{P}^{\prime}\left(p_{T^{\prime}}\right)\right)\left(\bigotimes_{v \in I^{e}(T) \cup I^{e}\left(T^{\prime}\right)} \bar{g}^{\prime \prime}(\widetilde{v}) \otimes \bigotimes_{w \in I^{o}(T) \cup I^{o}\left(T^{\prime}\right)} f^{\prime \prime}(\tilde{w})\right) \\
& =\left(h_{r}(l) \bar{\psi}_{r}^{T}\right) \circ_{i}\left(h_{s}(m) \bar{\psi}_{s}^{T^{\prime}}\right) .
\end{aligned}
$$

Here $\circ_{k}$ denotes either $\circ_{k}: \mathcal{O}(\tilde{u}) \otimes \mathcal{O}\left(\tilde{u^{\prime}}\right) \rightarrow \mathcal{O}([\tilde{e}])$ or $\circ_{k}: \mathcal{P}^{\prime}(\widetilde{u}) \otimes \mathcal{P}^{\prime}\left(\tilde{u^{\prime}}\right) \rightarrow \mathcal{P}^{\prime}([\tilde{e}])$. Moreover, in the first equation we use the inductive definition of $c_{i}^{r, s}(l, m)$ in Lemma 5.2, in the second equation we use the definition of $d_{i}^{r, s}\left(T, T^{\prime}\right)$ also in Lemma 5.2, in the third equation we use (b), in the fourth equation we use that $f^{\prime \prime}$ is an operad morphism, in the fifth equation we use the construction of operadic functors from operads in Proposition 3.10, and in the final equation we use (b) again. Furthermore, for simplicity we have omitted some symmetry isomorphisms in $\mathscr{V}$ in these equations.

The uniqueness of $h$ follows from the fact that the morphism $\bar{\psi}_{r}^{T}$ defined in Lemma 5.1 is related to the operadic functor of $\mathcal{P}$ by

$$
\bar{\psi}_{r}^{T}=\mathcal{P}\left(p_{T}\right)\left(\bigotimes_{v \in I^{e}(T)} \bar{g}^{\prime}(\widetilde{v}) \otimes \bigotimes_{w \in I^{o}(T)} f^{\prime}(\widetilde{w})\right) .
$$

Therefore, if $h^{\prime}: \mathcal{P} \rightarrow \mathcal{P}^{\prime}$ is an operad morphism satisfying $h^{\prime} f^{\prime}=f^{\prime \prime}$ and $h^{\prime} \bar{g}^{\prime}=\bar{g}^{\prime \prime}$, and if we denote $h_{r}^{\prime}(l)$ the composition of $h^{\prime}(l)$ with the canonical morphism to the colimit $P_{r}(l) \rightarrow \mathcal{P}(l)$, then the morphisms $h_{r}^{\prime}(l)$ must satisfy $h_{0}^{\prime}(l)=f^{\prime \prime}(l)$, and also (b) after replacing $h_{r}(m)$ with $h_{r}^{\prime}(m)$, therefore $h^{\prime}=h$ by the universal property of the pushouts $P_{r}(l)$ and the colimit $\mathcal{P}$.

\section{Proof of Theorem 1.1}

Assume in this section that $\mathscr{V}$ is also a cofibrantly generated monoidal model category (see Definition 4.2) satisfying the monoid axiom [20, Definition 3.3]. In order to explain what this means, let us recall some terminology from [15].

Given an ordinal $\lambda$, a directed diagram $X: \lambda \rightarrow \mathscr{V}$ is continuous if for any limit ordinal $\alpha<\lambda$, the canonical morphism

$$
\underset{i<\alpha}{\operatorname{colim}} X_{i} \longrightarrow X_{\alpha}
$$

is an isomorphism. The natural morphism from the first object to the colimit

$$
X_{0} \longrightarrow \underset{i<\lambda}{\operatorname{colim}} X_{i}
$$

is said to be the transfinite composition of the morphisms in the continuous diagram. We here do not exclude the possibility that $\lambda$ be finite. 
Given a class of morphisms $K$ in $\mathscr{V}$, a relative $K$-cell complex is a transfinite composition of morphisms $X: \lambda \rightarrow \mathscr{V}$ such that for any $i<\lambda$ with $i+1<\lambda$ the morphism $X_{i} \rightarrow X_{i+1}$ fits into a pushout diagram as follows, where the top horizontal arrow is in $K$ :

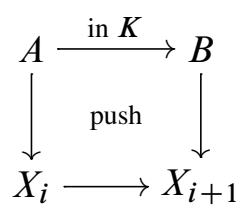

A plain $K$-cell complex is a relative $K$-cell complex with $X_{0}=0$ the initial object of $\mathscr{V}$. So far, nothing of this needs either the monoidal structure of $\mathscr{V}$ or its model category structure.

If $I$ and $J$ are sets of generating cofibrations and generating trivial cofibrations in $\mathscr{V}$, respectively, then the cofibrations in $\mathscr{V}$ are exactly the retracts of relative $I$-cell complexes, and the trivial cofibrations are the retracts of relative $J$-cell complexes. In particular the cofibrant objects in $\mathscr{V}$ are the retracts of $I$-cell complexes.

Definition 6.1 The monoid axiom for $\mathscr{V}$ says that, for

$$
K=\{f \otimes X ; f \text { is a trivial cofibration and } X \text { is an object in } \mathscr{V}\},
$$

all relative $K$-cell complexes are weak equivalences.

Proposition 6.2 Consider a pushout diagram in $\mathrm{Op}(\mathscr{V})$ as follows:

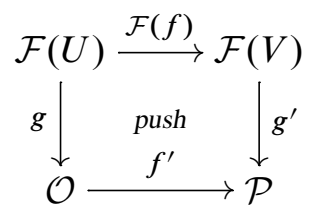

If $f$ is a trivial cofibration then $f^{\prime}(n): \mathcal{O}(n) \rightarrow \mathcal{P}(n)$ is a relative $K$-cell complex, $n \geq 0$, where $K$ is the class in the previous definition.

Proof By the pushout product axiom (Definition 4.2), the morphism (5) in Lemma 5.1 is the tensor product of a trivial cofibration and an object in $\mathscr{V}$, ie Equation $(5) \in K$. Therefore, by Theorem 5.4, $f^{\prime}(n)$ is a relative $K$-cell complex.

Consider the associated sets of generating cofibrations and generating trivial cofibrations in $\mathscr{V}^{\mathbb{N}}, I_{\mathbb{N}}$ and $J_{\mathbb{N}}$, respectively; see Remark 2.3.

Corollary 6.3 If $\mathscr{V}$ satisfies the monoid axiom, then a morphism in $\mathscr{V} \mathbb{N}$ underlying a relative $\mathcal{F}\left(J_{\mathbb{N}}\right)$-cell complex in $\operatorname{Op}(\mathscr{V})$ is a weak equivalence in $\mathscr{V} \mathbb{N}$. 
Now we are ready to prove Theorem 1.1.

Proof of Theorem 1.1 It is easy to see that operadic functors are the same as algebras over the monad associated to free operad adjunction in Section 5. Therefore, using the equivalence between operads and operadic functors in Proposition 3.10, one can easily show that the natural comparison functor from operads to algebras over the free operad monad is an equivalence of categories. Moreover, this monad preserves filtered colimits (see the explicit construction in Section 5), therefore the category $\mathrm{Op}(\mathscr{V})$ is complete and cocomplete [5, Proposition 4.3.6]. Furthermore, the forgetful functor $\mathrm{Op}(\mathscr{V}) \rightarrow \mathscr{V}^{\mathbb{N}}$ also preserves filtered colimits [5, Proposition 4.3.2], in particular, since $\mathcal{F}$ is a left adjoint and sources of morphisms in $I$ and $J$ are presentable in $\mathscr{V}$, then sources of morphisms in $\mathcal{F}\left(I_{\mathbb{N}}\right)$ and $\mathcal{F}\left(J_{\mathbb{N}}\right)$ are presentable in $\mathrm{Op}(\mathscr{V})$.

We can apply [20, Lemma 2.3] in order to prove the existence of the claimed model structure in $\mathrm{Op}(\mathscr{V})$. The smallness condition has already been checked, and condition (1) of [20, Lemma 2.3] has been established in Corollary 6.3.

Recall that a model category is right proper if the pullback of a weak equivalence along a fibration is again a weak equivalence [14, Definition 13.1.1 (2)]. The statement about right properness is obvious since fibrations and weak equivalences in $\operatorname{Op}(\mathscr{V})$ are detected by the forgetful functor $\mathrm{Op}(\mathscr{V}) \rightarrow \mathscr{V} \mathbb{N}$, and this functor is a right adjoint, so it preserves all limits, in particular pullbacks.

Recall also that a model category is combinatorial if it is cofibrantly generated and locally presentable. If $\mathscr{V}$ is combinatorial then $\mathrm{Op}(\mathscr{V})$ is locally presentable by $[1,2.3$ (1) and the Theorem in 2.78], hence it is combinatorial.

\section{Algebras}

In this section we recall the basic definitions about algebras in $\mathscr{C}$ over a nonsymmetric operad in $\mathscr{V}$ when $\mathscr{C}$ is appropriately enriched over $\mathscr{V}$; see [2, Section 1].

Assume we have a strong braided monoidal functor $\mathscr{V} \rightarrow Z(\mathscr{C})$, where $Z(\mathscr{C})$ is the center of $\mathscr{C}$, defined in [16]. Such a functor consists of an ordinary functor

$$
z: \mathscr{V} \longrightarrow \mathscr{b}
$$

together with natural isomorphisms

$$
\begin{aligned}
\text { multiplication: } z(X) \otimes z\left(X^{\prime}\right) & \longrightarrow z\left(X \otimes X^{\prime}\right), \\
\text { unit: } \mathbb{I}_{\mathscr{C}} & \longrightarrow z\left(\mathbb{I}_{\mathscr{V}}\right), \\
\zeta(X, Y): z(X) \otimes Y & \longrightarrow Y \otimes z(X),
\end{aligned}
$$


such that the multiplication and the unit satisfy well-known coherence laws [5, Definition 6.4.1] and the following three diagrams of isomorphisms commute:
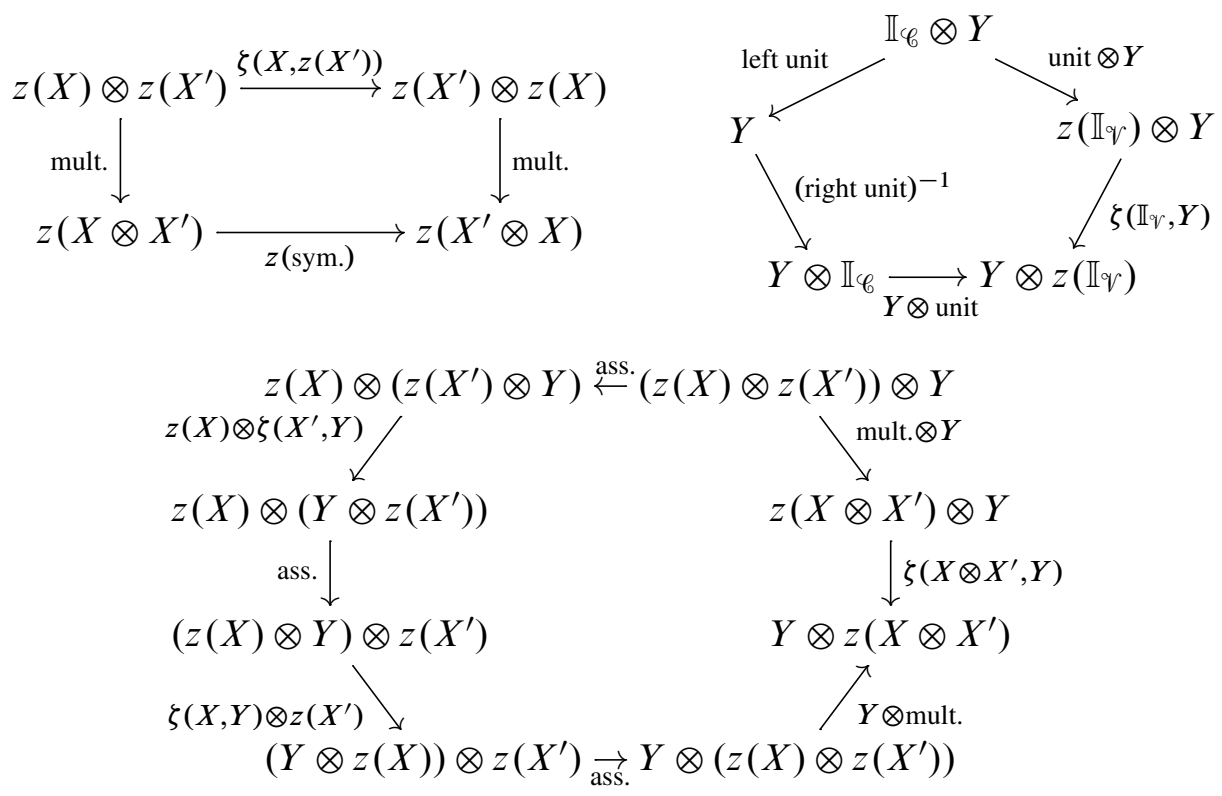

Moreover, suppose that the functor $z(-) \otimes Y: \mathscr{V} \rightarrow \mathscr{C}$ has a right adjoint

$$
\operatorname{Hom}_{\mathscr{C}}(Y,-): \mathscr{C} \longrightarrow \mathscr{V} \text {. }
$$

We will use the evaluation morphism

$$
\text { evaluation: } z\left(\operatorname{Hom}_{\mathscr{C}}(Y, Z)\right) \otimes Y \longrightarrow Z,
$$

which is the adjoint of the identity in $\operatorname{Hom}_{\mathscr{C}}(Y, Z)$.

Definition 7.1 The endomorphism operad of an object $Y$ in $\mathscr{C}$ is the nonsymmetric operad $\operatorname{End}_{\mathscr{C}}(Y)$ in $\mathscr{V}$ with

$$
\operatorname{End}_{\mathscr{C}}(Y)(n)=\operatorname{Hom}_{\mathscr{C}}(Y \otimes \stackrel{n}{*} \otimes Y, Y) .
$$

The unit

$$
u: \mathbb{I}_{\mathscr{V}} \longrightarrow \operatorname{End}_{\mathscr{C}}(Y)(1)
$$

is the adjoint of

$$
z\left(\mathbb{I}_{\mathscr{V}}\right) \otimes Y \stackrel{\text { unit }^{-1} \otimes Y}{\longrightarrow} \mathbb{I}_{\mathscr{C}} \otimes Y \stackrel{\text { left unit }}{\longrightarrow} Y .
$$

The composition laws, $1 \leq i \leq m, n \geq 0$,

$$
\circ_{i}: \operatorname{End}_{\mathscr{C}}(Y)(m) \otimes \operatorname{End}_{\mathscr{C}}(Y)(n) \longrightarrow \operatorname{End}_{\mathscr{C}}(Y)(m+n-1)
$$


are the adjoints of

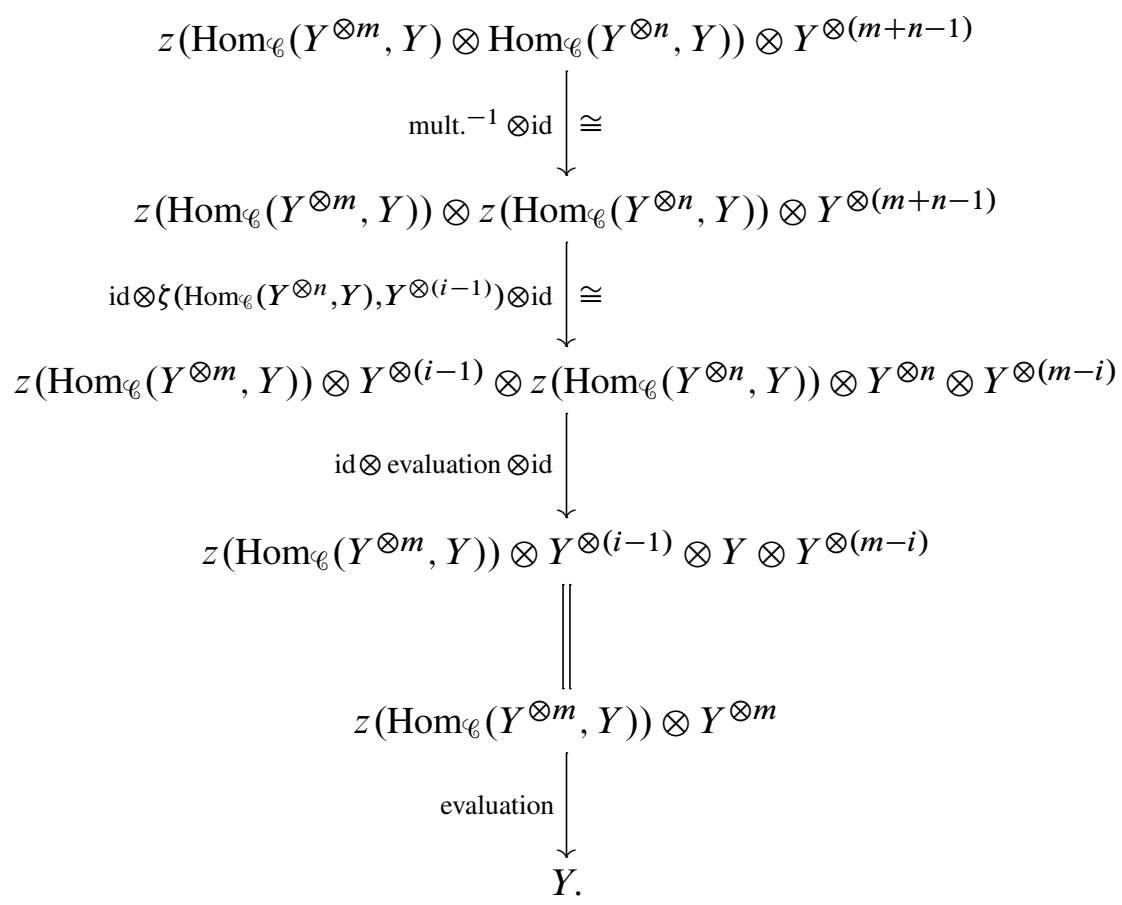

Here we have omitted some obvious associativity isomorphisms in $\mathscr{C}$.

Given a nonsymmetric operad $\mathcal{O}$ in $\mathscr{V}$, an $\mathcal{O}$-algebra in $\mathscr{b}$ is an object $Y$ in $\mathscr{b}$ together with an operad morphism $\mathcal{O} \rightarrow \operatorname{End}_{\mathfrak{C}}(Y)$.

Equivalently, an $\mathcal{O}$-algebra structure on $Y$ is given by morphisms in $\mathscr{C}, n \geq 0$,

$$
v_{n}: z(\mathcal{O}(n)) \otimes Y^{\otimes n} \longrightarrow Y,
$$

such that the following diagrams commute, $1 \leq i \leq m, n \geq 0$ :

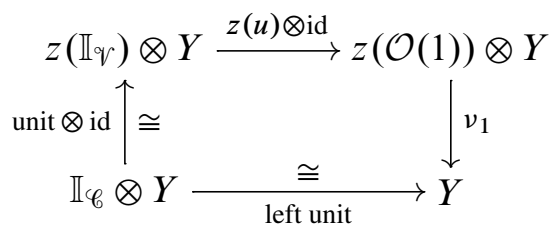




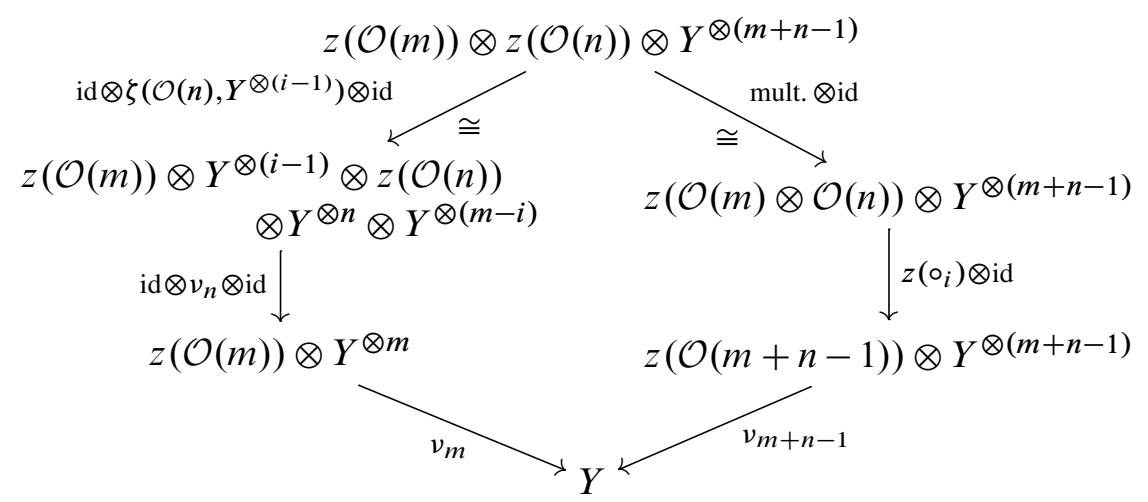

An $\mathcal{O}$-algebra morphism $f: Y \rightarrow Z$ is a morphism in $\mathscr{C}$ such that the following squares commute, $n \geq 0$ :

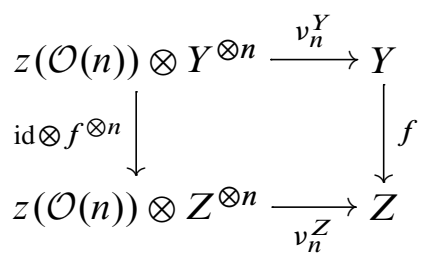

The category of $\mathcal{O}$-algebras in $\mathscr{C}$ will be denoted by $\operatorname{Alg}_{\mathscr{C}}(\mathcal{O})$.

Remark 7.2 The initial $\mathcal{O}$-algebra in $\mathscr{C}$ is $z(\mathcal{O}(0))$ with structure morphisms

$$
v_{n}: z(\mathcal{O}(n)) \otimes z(\mathcal{O}(0))^{\otimes n} \stackrel{\text { mult. }}{\cong} z\left(\mathcal{O}(n) \otimes \mathcal{O}(0)^{\otimes n}\right) \stackrel{z\left(\mu_{n ; 0, n, 0}\right)}{\longrightarrow} z(\mathcal{O}(0)) .
$$

Here we use the convention $\mu_{0 ; \varnothing}=\operatorname{id}_{\mathcal{O}(0)}$. If $A$ is an $\mathcal{O}$-algebra, the structure morphism $v_{0}^{A}: z(\mathcal{O}(0)) \rightarrow A$ is the unique morphism of $\mathcal{O}$-algebras $z(\mathcal{O}(0)) \rightarrow A$. The final $\mathcal{O}$-algebra in $\mathscr{C}$ is the final object of $\mathscr{C}$ endowed with the only possible $\mathcal{O}-$ algebra structure.

\section{The relevant algebra pushout}

Assume we are in the same circumstances as in the previous section. Let $\mathcal{O}$ be a nonsymmetric operad in $\mathscr{V}$. The functor $\operatorname{Alg}_{\mathscr{b}}(\mathcal{O}) \rightarrow \mathscr{C}$ forgetting the $\mathcal{O}$-algebra structure has a left adjoint

$$
\mathcal{F}_{\mathcal{O}}: \mathscr{C} \longrightarrow \operatorname{Alg}_{\mathscr{C}}(\mathcal{O})
$$

the free $\mathcal{O}$-algebra functor, explicitly defined as (compare [2, Proposition 1.3])

$$
\mathcal{F}_{\mathcal{O}}(Y)=\coprod_{p \geq 0} z(\mathcal{O}(p)) \otimes Y^{\otimes p}
$$


The action of $\mathcal{O}$ on $\mathcal{F}_{\mathcal{O}}(Y)$,

$$
\begin{aligned}
& z(\mathcal{O}(n)) \otimes \mathcal{F}_{\mathcal{O}}(Y)^{\otimes n}=z(\mathcal{O}(n)) \otimes \bigotimes_{i=1}^{n}\left(\coprod_{p_{i} \geq 0} z\left(\mathcal{O}\left(p_{i}\right)\right) \otimes Y^{\otimes p_{i}}\right) \\
& \begin{array}{l}
\cong \bigsqcup_{n} z(\mathcal{O}(n)) \otimes \bigotimes_{i=1}^{n}\left(z\left(\mathcal{O}\left(p_{i}\right)\right) \otimes Y^{\otimes p_{i}}\right) \\
\cong \coprod_{p_{1}, \ldots, p_{n} \geq 0} z(\mathcal{O}(n)) \otimes z\left(\mathcal{O}\left(p_{1}\right)\right) \otimes \cdots \otimes z\left(\mathcal{O}\left(p_{n}\right)\right) \otimes Y^{\otimes \sum_{i=1}^{n} p_{i}} \\
\quad{ }_{p_{1}, \ldots, p_{n} \geq 0} \\
\cong \coprod_{p_{1}, \ldots, p_{n} \geq 0} z\left(\mathcal{O}(n) \otimes \mathcal{O}\left(p_{1}\right) \otimes \cdots \mathcal{O}\left(p_{n}\right)\right) \otimes Y^{\otimes \sum_{i=1}^{n} p_{i}} \\
\mathcal{F}_{\mathcal{O}}(Y)=\coprod_{p \geq 0} z(\mathcal{O}(p)) \otimes Y^{\otimes p},
\end{array}
\end{aligned}
$$

is defined as the morphism which sends the factor $\left(p_{1}, \ldots, p_{n}\right) \in \mathbb{N}^{n}$ in the source to the factor $p=p_{1}+\cdots+p_{n} \in \mathbb{N}$ in the target via $z\left(\mu_{n ; p_{1}, \ldots, p_{n}}\right) \otimes \mathrm{id}, n \geq 1$. For $n=0$, the morphism $v_{0}: z(\mathcal{O}(0)) \rightarrow \mathcal{F}_{\mathcal{O}}(Y)$ is the inclusion of the factor $p=0$ of the coproduct.

The unit of the adjunction is the following composite morphism in $\mathscr{b}$ :

$$
Y \stackrel{\text { (left unit) }^{-1}}{\cong} \mathbb{I}_{\mathscr{C}} \otimes Y \stackrel{\text { unit } \otimes \text { id }}{\cong} z\left(\mathbb{I}_{\mathscr{V}}\right) \otimes Y \stackrel{z(u) \otimes \text { id }}{\longrightarrow} z(\mathcal{O}(1)) \otimes Y \stackrel{\begin{array}{c}
\text { inclusion of } \\
\text { the factor } p=1
\end{array}}{\longrightarrow} \mathcal{F}_{\mathcal{O}}(Y) .
$$

Moreover, given an $\mathcal{O}$-algebra $A$, the counit of the adjunction is defined by the multiplication morphisms in Equation (13):

$$
\left(v_{p}\right)_{p \geq 0}: \mathcal{F}_{\mathcal{O}}(A) \longrightarrow A .
$$

In this section we give an explicit construction of the pushout of two morphisms in $\operatorname{Alg}_{\mathscr{C}}(\mathcal{O})$ as follows:

$$
A \stackrel{g}{\longleftarrow} \mathcal{F}_{\mathcal{O}}(Y) \stackrel{\mathcal{F}_{\mathcal{O}}(f)}{\longrightarrow} \mathcal{F}_{\mathcal{O}}(Z)
$$

Consider the adjoint diagram in $\mathscr{b}$ :

$$
A \stackrel{\bar{g}}{\longleftarrow} Y \stackrel{f}{\longrightarrow} Z .
$$

The pushout of Equation (14) is an $\mathcal{O}$-algebra $B$ together with morphisms $f^{\prime}: A \rightarrow B$ in $\operatorname{Alg}_{\mathscr{C}}(\mathcal{O})$ and $\bar{g}^{\prime}: Z \rightarrow B$ in $\mathscr{C}$ such that $f^{\prime} \bar{g}=\bar{g}^{\prime} f$ in $\mathscr{C}$. Moreover, given an 
$\mathcal{O}$-algebra $B^{\prime}$ and morphisms $f^{\prime \prime}: A \rightarrow B^{\prime}$ in $\operatorname{Alg}_{\mathscr{C}}(\mathcal{O})$ and $\bar{g}^{\prime \prime}: Z \rightarrow \mathcal{P}^{\prime}$ in $\mathscr{C}$ with $f^{\prime \prime} \bar{g}=\bar{g}^{\prime \prime} f$ in $\mathscr{C}$, there is a unique morphism $h: B \rightarrow B^{\prime}$ in $\operatorname{Alg}_{\mathscr{C}}(\mathcal{O})$ such that $f^{\prime \prime}=h f^{\prime}$ and $\bar{g}^{\prime \prime}=h \bar{g}^{\prime}$ in $\mathscr{C}$.

The following lemma allows an inductive definition of the pushout of Equation (14) as an object in $\mathscr{b}$. We omit proofs in this section since the results are simpler analogs of those in Section 5, and the proofs follow very much the same steps.

Lemma 8.1 There is a sequence in $\mathscr{b}$,

$$
A=B_{0} \stackrel{\varphi_{1}}{\longrightarrow} B_{1} \rightarrow \cdots \rightarrow B_{t-1} \stackrel{\varphi_{t}}{\longrightarrow} B_{t} \rightarrow \cdots,
$$

where the morphism $\varphi_{t}$ is the pushout of

$$
\coprod_{n \geq 1} \coprod_{\substack{S \subset\{1, \ldots, n\} \\ \operatorname{card}(S)=t}} z(\mathcal{O}(n)) \otimes k_{1}^{S} \odot \cdots \odot k_{n}^{S}, \quad k_{i}^{S}= \begin{cases}f & i \in S, \\ 0 \rightarrow A & i \notin S,\end{cases}
$$

along the unique morphism

$$
\left(\psi_{t}^{n, S}\right)_{n, S}: \coprod_{n \geq 1} \coprod_{\substack{S \subset\{1, \ldots, n\} \\ \operatorname{card}(S)=t}} z(\mathcal{O}(n)) \otimes s\left(k_{1}^{S} \odot \cdots \odot k_{n}^{S}\right) \longrightarrow B_{t-1},
$$

such that for $t=1$ and $1 \leq i \leq n$,

$$
\psi_{1}^{n,\{i\}}=v_{n}\left(\mathrm{id}_{z(\mathcal{O}(n))} \otimes \mathrm{id}^{\otimes(i-1)} \otimes \bar{g} \otimes \mathrm{id}^{\otimes(n-i)}\right),
$$

and for $t>1$ and $i \in S$,

$$
\psi_{t}^{n, S}\left(\mathrm{id}_{z(\mathcal{O}(n))} \otimes \kappa_{i}\right)=\bar{\psi}_{t-1}^{n, S \backslash\{i\}}\left(\mathrm{id}_{z(\mathcal{O}(n))} \otimes \mathrm{id}^{\otimes(i-1)} \otimes \bar{g} \otimes \mathrm{id}^{\otimes(n-i)}\right) .
$$

Here $\left(\bar{\psi}_{t-1}^{n, S^{\prime}}\right)_{n, S^{\prime}}$ denotes the pushout of $\left(\psi_{t-1}^{n, S^{\prime}}\right)_{n, S^{\prime}}$, ie Equation (16) for $t-1$, along Equation (15).

We now endow

$$
B=\operatorname{colim}_{t \geq 0} B_{t}
$$

with an $\mathcal{O}$-algebra structure.

Lemma 8.2 There are unique morphisms in $\mathscr{C}$,

$$
c_{n}^{t_{1}, \ldots, t_{n}}: z(\mathcal{O}(n)) \otimes B_{t_{1}} \otimes \cdots \otimes B_{t_{n}} \longrightarrow B_{t_{1}+\cdots+t_{n}}, \quad n \geq 1, t_{i} \geq 0,
$$


such that

$$
c_{n}^{0, \stackrel{n}{\prime}, 0}=v_{n}: z(\mathcal{O}(n)) \otimes A^{\otimes n} \longrightarrow A,
$$

and, with the convention $\bar{\psi}_{0}^{p_{i}, S_{i}}=v_{p_{i}}$, if $S_{i} \subset\left\{1, \ldots, p_{i}\right\}$ is a subset of cardinality card $S_{i}=t_{i}, 1 \leq i \leq n$, then

$c_{n}^{t_{1}, \ldots, t_{i}, \ldots, t_{n}}\left(\mathrm{id}^{\otimes(i-1)} \otimes \varphi_{t_{i}} \otimes \mathrm{id}^{\otimes(n-i)}\right)=\varphi_{t_{1}+\cdots+t_{n}} c_{n}^{t_{1}, \ldots, t_{i}-1, \ldots, t_{n}}$,

$c_{n}^{t_{1}, \ldots, t_{n}}\left(\bar{\psi}_{t_{1}}^{p_{1}, S_{1}} \otimes \cdots \otimes \bar{\psi}_{t_{n}}^{p_{n}, S_{n}}\right)$

$$
=\bar{\psi}_{t_{1}+\cdots+t_{n}}^{p_{1}+\cdots+p_{n}, \cup_{i=1}^{n}\left(S_{i}+\left(p_{1}+\cdots+p_{i-1}\right)\right)}\left(z\left(\mu_{n ; p_{1}, \ldots, p_{n}}\right) \otimes \mathrm{id}^{\otimes \sum_{i=1}^{n} p_{i}}\right) .
$$

Here $S+p=\{i+p ; i \in S\}$ and $\mu$ is the multiplication of the operad $\mathcal{O}$. For simplicity, in these equations we have omitted some obvious structure isomorphisms of $\mathscr{V}, \mathscr{b}$ and $z$.

We define

$$
f^{\prime}: A=B_{0} \longrightarrow \underset{t \geq 0}{\operatorname{colim}} B_{t}=B
$$

as the canonical morphism to the colimit. Moreover, for $n \geq 1$ we define

$$
v_{n}^{B}: z(\mathcal{O}(n)) \otimes B^{\otimes n} \longrightarrow B
$$

as the colimit of the morphisms $c_{n}^{t_{1}, \ldots, t_{n}}$ in the previous lemma, $t_{i} \geq 0$, and for $n=0$,

$$
v_{0}^{B}: z(\mathcal{O}(0)) \stackrel{v_{0}^{A}}{\longrightarrow} A \stackrel{f^{\prime}}{\longrightarrow} B .
$$

Furthermore, we define $\bar{g}^{\prime}: Z \rightarrow B$ as the composite morphism

$$
Z \stackrel{\text { (left unit) })^{-1}}{\cong} \mathbb{I}_{\mathscr{C}} \otimes Z \stackrel{\text { unit } \otimes \mathrm{id}}{\cong} z\left(\mathbb{I}_{\mathscr{V}}\right) \otimes Z \stackrel{z(u) \otimes \mathrm{id}}{\longrightarrow} z(\mathcal{O}(1)) \otimes Z \stackrel{\bar{\psi}_{1}^{1,\{1\}} \longrightarrow}{\longrightarrow} B_{1} \stackrel{\begin{array}{c}
\text { projection to } \\
\text { the colimit }
\end{array}}{\longrightarrow} B .
$$

Theorem 8.3 The morphisms $v_{n}^{B}, n \geq 0$, define an $\mathcal{O}$-algebra structure on $B$, $f^{\prime}: A \rightarrow B$ is an $\mathcal{O}$-algebra morphism, and if $g^{\prime}: \mathcal{F}_{\mathcal{O}}(Z) \rightarrow B$ is the adjoint of $\bar{g}^{\prime}: Z \rightarrow B$, then the following square is a pushout in $\operatorname{Alg}_{\mathscr{C}}(\mathcal{O})$ :

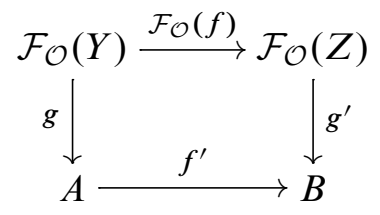




\section{Proofs of Theorem 1.2 and Theorem 1.3}

Suppose that we are in the same conditions as in the two previous sections. Assume also that $\mathscr{V}$ and $\mathscr{C}$ are monoidal model categories (see Definition 4.2) and that the composite functor

$$
\mathscr{V} \stackrel{z}{\longrightarrow} Z(\mathscr{b}) \stackrel{\text { forget }}{\longrightarrow} \mathscr{C}
$$

is a left Quillen functor [15, Definition 1.3.1]. We will need a nonsymmetric version of the monoid axiom in Definition 6.1.

Definition 9.1 The monoid axiom for $\mathscr{b}$ says that, for

$$
\begin{aligned}
K^{\prime}=\left\{f_{1} \odot \cdots \odot f_{n} ;\right. & n \geq 1, S \subset\{1, \ldots, n\} \text { is a subset with card } S \geq 1, \\
& f_{i} \text { is a trivial cofibration if } i \in S, \\
& \left.f_{i}: 0 \rightarrow X_{i} \text { for some object } X_{i} \text { in } \mathscr{b} \text { if } i \notin S\right\},
\end{aligned}
$$

all relative $K^{\prime}$-cell complexes are weak equivalences.

Notice that, as a consequence of the pushout product axiom, this is indeed equivalent to the monoid axiom in Definition 6.1 when $\mathscr{C}$ is symmetric. In any case, if all objects in $\mathscr{b}$ are cofibrant then the monoid axiom is a consequence of the pushout product axiom.

Suppose from now on that $\mathscr{C}$ satisfies the monoid axiom and is cofibrantly generated with sets of generating cofibrations and generating trivial cofibrations $I$ and $J$, respectively, with presentable sources.

Proposition 9.2 Consider a pushout diagram in $\operatorname{Alg}_{\mathscr{b}}(\mathcal{O})$ as follows.

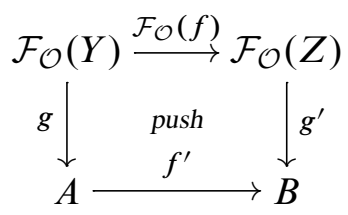

(1) If $f$ is a trivial cofibration in $\mathscr{C}$, then the underlying morphism $f^{\prime}: A \rightarrow B$ in $\mathscr{C}$ is a relative $K^{\prime}$-cell complex, where $K^{\prime}$ is the class in Definition 9.1.

(2) Suppose $A$ is cofibrant in $\mathscr{C}, f$ is a cofibration in $\mathscr{C}$, and $\mathcal{O}(n)$ is cofibrant in $\mathscr{V}, n \geq 0$. Then the morphism $f^{\prime}: A \rightarrow B$ is a cofibration in $\mathscr{C}$, in particular $B$ is cofibrant in $\mathscr{b}$. 
Proof In case (1), the morphism Equation (15) in Lemma 8.1 is in $K^{\prime}$, hence (1) follows from Theorem 8.3.

In case (2), since $z$ is a left Quillen functor, the objects $z(\mathcal{O}(n))$ are cofibrant in $\mathscr{b}$. Therefore, by the pushout product axiom (Definition 4.2) the morphism Equation (15) is a cofibration in $\mathscr{C}$. Furthermore, by Theorem 8.3 the morphism $f^{\prime}: A \rightarrow B$ is a transfinite composition of cofibrations in $\mathscr{C}$, hence a cofibration in $\mathscr{C}$ itself [14, Proposition 10.3.4].

As an immediate consequence of (1) here and the monoid axiom, we obtain the following.

Corollary 9.3 A morphism in $\mathscr{C}$ underlying a relative $\mathcal{F}_{\mathcal{O}}(J)$-cell complex in $\operatorname{Alg}_{\mathscr{C}}(\mathcal{O})$ is a weak equivalence in $\mathscr{C}$.

Now we are ready to prove Theorem 1.2.

Proof of Theorem 1.2 Using the explicit description of the free operad adjunction at the beginning of Section 8 , it is easy to see that $\mathcal{O}$-algebras are the same thing as algebras over the monad associated to the free $\mathcal{O}$-algebra adjunction; compare [2, Proposition 1.3]. Moreover, this monad preserves filtered colimits (see again the explicit construction), therefore the category $\operatorname{Alg}_{\mathscr{C}}(\mathcal{O})$ is complete and cocomplete [5, Proposition 4.3.6]. Furthermore, the forgetful functor $\operatorname{Alg}_{\mathscr{C}}(\mathcal{O}) \rightarrow \mathscr{C}$ also preserves filtered colimits [5, Proposition 4.3.2], in particular, since $\mathcal{F}_{\mathcal{O}}$ is a left adjoint and sources of morphisms in $I$ and $J$ are presentable in $\mathscr{C}$, then sources of morphisms in $\mathcal{F}_{\mathcal{O}}(I)$ and $\mathcal{F}_{\mathcal{O}}(J)$ are presentable in $\operatorname{Alg}_{\mathscr{C}}(\mathcal{O})$.

We can apply [20, Lemma 2.3] in order to prove the existence of the claimed model structure in $\operatorname{Alg}_{\mathscr{b}}(\mathcal{O})$. The smallness condition has already been checked, and condition (1) of [20, Lemma 2.3] has been established in Corollary 9.3.

The statement about right properness is obvious since fibrations and weak equivalences in $\operatorname{Alg}_{\mathscr{C}}(\mathcal{O})$ are detected by the forgetful functor $\operatorname{Alg}_{\mathscr{C}}(\mathcal{O}) \rightarrow \mathscr{C}$, and this functor is a right adjoint, so it preserves all limits, in particular pullbacks.

If $\mathscr{C}$ is combinatorial then $\operatorname{Alg}_{\mathscr{C}}(\mathcal{O})$ is locally presentable by [1, $2.3(1)$ and the Theorem in 2.78], hence it is combinatorial.

Lemma 9.4 Suppose that $\mathcal{O}$ is an operad in $\mathscr{V}$ with $\mathcal{O}(n)$ cofibrant for all $n \geq 0$. Then any cofibrant $\mathcal{O}$-algebra is also cofibrant as an object in $\mathscr{C}$. 
Proof Cofibrant $\mathcal{O}$-algebras are retracts of $\mathcal{F}_{\mathcal{O}}(I)$-cell complexes, and cofibrant objects in $\mathscr{C}$ are closed under retracts, so it is enough to check that $\mathcal{F}_{\mathcal{O}}(I)$-cell complexes are cofibrant in $\mathscr{C}$. The initial $\mathcal{O}$-algebra in $\mathscr{C}$ (see Remark 7.2) is cofibrant in $\mathscr{C}$, since $\mathcal{O}(0)$ is cofibrant in $\mathscr{V}$ and $z$ is a left Quillen functor. Using Proposition 9.2 (2), an induction argument proves that any $\mathcal{F}_{\mathcal{O}}(I)$-cell complex is cofibrant in $\mathscr{C}$.

Corollary 9.5 Let $\mathcal{O}$ be an operad in $\mathscr{V}$ with $\mathcal{O}(n)$ cofibrant for all $n \geq 0$. Then, the forgetful functor $\operatorname{Alg}_{\mathscr{C}}(\mathcal{O}) \rightarrow \mathscr{C}$ preserves cofibrations with cofibrant source.

Proof This is an immediate consequence of Lemma 9.4 and Proposition 9.2 (2), since cofibrations in $\operatorname{Alg}_{\mathscr{C}}(\mathcal{O})$ are retracts of relative $\mathcal{F}_{\mathcal{O}}(I)$-cell complexes, the forgetful functor preserves filtered colimits, and cofibrations in $\mathscr{b}$ are closed under transfinite compositions and retracts.

Lemma 9.6 Under the hypotheses of Theorem 1.3, suppose that we have a pushout diagram in $\operatorname{Alg}_{\mathscr{C}}(\mathcal{O})$,

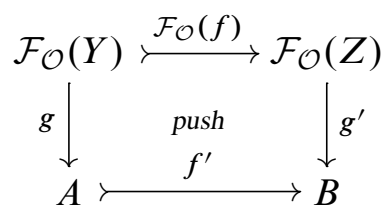

where $f$ is a cofibration in $\mathscr{b}$ and $A$ is a cofibrant $\mathcal{O}$-algebra. If the unit of the adjunction evaluated at $A$ is a weak equivalence $\eta_{A}: A \stackrel{\sim}{\rightarrow} \phi^{*} \phi_{*} A$, then it is also a weak equivalence when evaluated at $B, \eta_{B}: B \stackrel{\sim}{\rightarrow} \phi^{*} \phi_{*} B$.

Proof Since $\phi_{*}$ is left adjoint to $\phi^{*}$, which is the identity on the underlying object in $\mathscr{C}$, there is a natural isomorphism $\phi_{*} \mathcal{F}_{\mathcal{O}} \cong \mathcal{F}_{\mathcal{P}}$ that we regard as an identification, and the morphism $\phi_{*}\left(f^{\prime}\right)$ fits into the following pushout diagram in $\operatorname{Alg}_{\mathscr{C}}(\mathcal{P})$ :

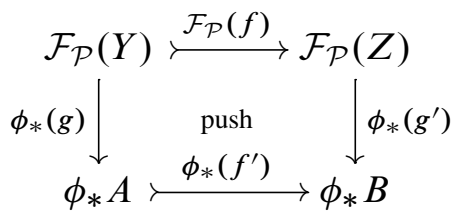

The $\mathcal{O}$-algebra $A$ is cofibrant and $\phi_{*}$ is a left Quillen functor, therefore $\phi_{*} A$ is a cofibrant $\mathcal{P}$-algebra, in particular, both $A$ and $\phi_{*} A$ are cofibrant in $\mathscr{C}$ by Lemma 9.4. Notice that the underlying object of $\phi_{*} A$ and $\phi^{*} \phi_{*} A$ in $\mathscr{C}$ is the same. 
Let us call $C=\phi^{*} \phi_{*} B$. By Lemma 8.1, the morphism in $\mathscr{C}$ underlying $\eta_{B}$ is the colimit in $t \in \mathbb{N}$ of an inductively constructed diagram of cofibrant objects in $\mathscr{C}, t>0$,

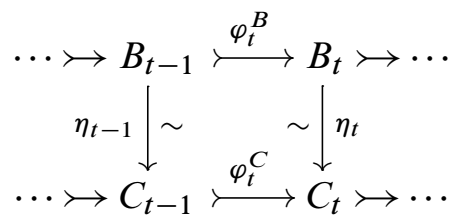

such that $B_{0}=A, C_{0}=\phi^{*} \phi_{*} A, \eta_{0}=\eta_{A}$, the morphism $\eta_{t}$ is the pushout of the horizontal lines of the following diagram

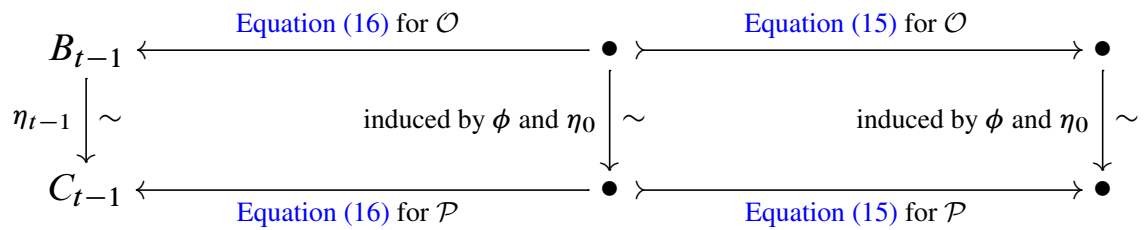

and $\varphi_{t}^{B}$ and $\varphi_{t}^{C}$ are the natural morphisms to the pushout.

The objects $\mathcal{O}(n)$ and $\mathcal{P}(n)$ are cofibrant in $\mathscr{V}$ and $z$ is a left Quillen functor, hence $z(\mathcal{O}(n))$ and $z(\mathcal{P}(n))$ are cofibrant in $\mathscr{C}, n \geq 0$. Moreover, $f$ is a cofibration in $\mathscr{C}$ and $A$ and $\phi^{*} \phi_{*} A$ are cofibrant in $\mathscr{C}$. Therefore Lemma 4.4 shows that the square on the right has weak equivalences in the columns and cofibrations in the rows. In particular, $\varphi_{t}^{B}$ and $\varphi_{t}^{C}$ are cofibrations in $\mathscr{C}$ and, by the gluing property in left proper model categories [14, Proposition 13.5.4], $\eta_{t}$ is a weak equivalence in $\mathscr{C}$.

To conclude, $\eta_{B}=\operatorname{colim}_{t \geq 0} \eta_{t}$ is a weak equivalence in $\mathscr{b}$ since (a) is a weak equivalence between cofibrant objects in the Reedy model category of directed diagrams in $\mathscr{C}$ indexed by $\mathbb{N}$ [15, Theorem 5.1.3] and Ken Brown's lemma [15, Lemma 1.1.12] applies, because colim $_{t \geq 0}$ is a left Quillen functor [15, Corollary 5.1.6].

Finally, we are ready to prove Theorem 1.3.

Proof of Theorem 1.3 We will use the criterion in [15, Corollary 1.3.16 (c)] to detect Quillen equivalences. The functor $\phi^{*}$ preserves and reflects weak equivalences, since it is the identity on the underlying object in $\mathscr{C}$. Therefore, it is enough to check that the unit of the adjunction $\eta_{A}: A \rightarrow \phi^{*} \phi_{*} A$ is a weak equivalence for any cofibrant $\mathcal{O}$-algebra $A$.

Weak equivalences are closed under retracts and cofibrant $\mathcal{O}$-algebras are retracts of $\mathcal{F}_{\mathcal{O}}(I)$-cell complexes, so we can suppose that $A$ is an $\mathcal{F}_{\mathcal{O}}(I)$-cell complex, $A=\operatorname{colim}_{i<\lambda} A_{i}$. We now proceed by induction on the ordinal $\lambda$. 
For $\lambda=1, A$ is the initial $\mathcal{O}$-algebra; see Remark 7.2. Then $\phi_{*} A$ is the initial $\mathcal{P}$-algebra, since $\phi_{*}$ is a left adjoint, and $\eta_{A}=z(\phi(0)): z(\mathcal{O}(0)) \rightarrow z(\mathcal{P}(0))$. The morphism $\phi(0)$ is a weak equivalence between cofibrant objects in $\mathscr{V}$ and $z$ is a left Quillen functor, therefore $z(\phi(0))$ is also a weak equivalence between cofibrant objects in $\mathscr{C}$ by [15, Lemma 1.1.12].

If $\lambda=\alpha+1$ and the result is true for $\alpha$, then it is also true for $\lambda$ by the previous lemma.

Suppose now that $\lambda$ is a limit ordinal and that the result is true for all $i<\lambda$. The functor $\phi_{*}$ preserves colimits, since it is a left adjoint, and $\phi^{*}$ preserves filtered colimits, because it is the identity over $\mathscr{C}$ and forgetful functors from algebras to $\mathscr{C}$ preserve filtered colimits. In particular $\eta_{A}=\operatorname{colim}_{i<\lambda} \eta_{i}$ is a colimit of weak equivalences by induction hypothesis. By Proposition 9.2 (2), an $\mathcal{F}_{\mathcal{O}}(I)$-cell complex is a colimit of a continuous diagram of cofibrations between cofibrant objects in $\mathscr{C}$, and the same is true for $\mathcal{F}_{\mathcal{P}}(I)$-cell complexes. This applies to $A$ and $\phi_{*} A$. Such diagrams are cofibrant objects in Reedy model categories of directed diagrams in $\mathscr{C}$ [15, Theorem 5.1.3]. Therefore, $\eta_{A}$ is the colimit of a weak equivalence between cofibrant objects in the Reedy model category of directed diagrams in $\mathscr{C}$ indexed by $\lambda$. Now, Ken Brown's lemma [15, Lemma 1.1.12] shows that $\eta_{A}$ is a weak equivalence, since $\operatorname{colim}_{i<\lambda}$ is a left Quillen functor [15, Corollary 5.1.6].

\section{An application to enriched categories and $A_{\infty}$-categories}

In this section we lay the foundations to construct model categories of categorified algebraic structures. This is applied to enriched categories and enriched $A_{\infty}$-categories.

Definition 10.1 Given a set $S$, a $\mathscr{V}$-graph $M$ with object set $S$ is a collection of objects in $\mathscr{V}$ indexed by $S \times S, M=\{M(x, y)\}_{x, y \in S}$. The category $\operatorname{Graph}_{S}(\mathscr{V})$ of $\mathscr{V}$-graphs with object set $S$, where morphisms are defined in the obvious way, is biclosed monoidal with tensor product

$$
(M \otimes S N)(x, y)=\coprod_{z \in S} M(z, y) \otimes N(x, z) .
$$

The unit object $\mathbb{I}_{S}$ is

$$
\mathbb{I}_{S}(x, y)= \begin{cases}\mathbb{I} & \text { the monoidal unit of } \mathscr{V}, \text { if } x=y, \\ 0 & \text { the initial object of } \mathscr{V}, \text { if } x \neq y\end{cases}
$$


This monoidal category is clearly nonsymmetric, unless $S$ is a singleton. The right adjoint of $M \otimes-$ is the functor $\operatorname{Hom}_{l}^{S}(M,-)$ defined as

$$
\operatorname{Hom}_{l}^{S}(M, P)(x, y)=\prod_{z \in S} \operatorname{Hom}(M(y, z), P(x, z)),
$$

and the right adjoint of $-\otimes N$ is the functor $\operatorname{Hom}_{r}^{S}(N,-)$ defined as

$$
\operatorname{Hom}_{r}^{S}(N, P)(x, y)=\prod_{z \in S} \operatorname{Hom}(N(z, x), P(z, y)) .
$$

We have a strong braided monoidal functor $z: \mathscr{V} \rightarrow \operatorname{Graph}_{S}(\mathscr{V})$ defined as

$$
z(A)(x, y)= \begin{cases}A & \text { if } x=y \\ 0 & \text { if } x \neq y\end{cases}
$$

Moreover,

$$
\left(z(A) \otimes_{S} M\right)(x, y)=A \otimes M(x, y), \quad(M \otimes S z(A))(x, y)=M(x, y) \otimes A,
$$

and the natural isomorphism

$$
\zeta(A, M): z(A) \otimes_{S} M \cong M \otimes_{S} z(A),
$$

is defined as the symmetry isomorphism of $\mathscr{V}$ coordinatewise.

Remark 10.2 If $\mathscr{V}$ is a model category, the category $\operatorname{Graph}_{S}(\mathscr{V})$ inherits from $\mathscr{V}$ a product model category structure, where fibrations, cofibrations and weak equivalences are defined coordinatewise. If $\mathscr{V}$ is cofibrantly generated (resp. combinatorial) then so is $\operatorname{Graph}_{S}(\mathscr{V})$; compare Remark 2.3. Moreover, since $S \times S$ is a set, a $\mathscr{V}$-graph $M$ is presentable provided $M(x, y)$ is presentable for all $x, y \in S$. In particular, if $\mathscr{V}$ has sets of generating cofibrations and generating trivial cofibrations with presentable source, then so does $\operatorname{Graph}_{\mathscr{V}}(S)$. Furthermore, if $\mathscr{V}$ is right proper then the product model category $\operatorname{Graph}_{\mathscr{V}}(S)$ is also right proper.

Notice that the composite functor $\mathscr{V} \stackrel{z}{\rightarrow} Z\left(\operatorname{Graph}_{S}(\mathscr{V})\right) \rightarrow \operatorname{Graph}_{S}(\mathscr{V})$ preserves fibrations, cofibrations and weak equivalences, and it has a right adjoint defined by

$$
M \mapsto \prod_{x \in S} M(x, x) .
$$

This adjoint pair is therefore a Quillen adjunction.

Proposition 10.3 If $\mathscr{V}$ satisfies the monoid axiom then $\operatorname{Graph}_{S}(\mathscr{V})$ also satisfies the monoid axiom. 
Proof It is enough to notice, using the symmetry of $\mathscr{V}$ and the pushout product axiom in $\mathscr{V}$, that any morphism $f_{1} \odot \cdots \odot f_{n}$ in the class of morphisms $K^{\prime}$ of $\operatorname{Graph}_{S}(\mathscr{V})$ in Definition 9.1 is componentwise a morphism in the class $K$ of $\mathscr{V}$ in Definition 6.1.

Categories enriched on $\mathscr{V}$ with set of objects $S$ are the same as monoids in $\operatorname{Graph}_{S}(\mathscr{V})$. These monoids are the same as algebras over the nonsymmetric operad $\mathrm{Ass}^{\mathcal{V}}$ in $\mathscr{V}$ defined by $\operatorname{Ass}^{\mathcal{V}}(n)=\mathbb{I}, n \geq 0$. All compositions in $\mathrm{Ass}^{\mathcal{V}}$ are unit isomorphisms $\mathbb{I} \otimes \mathbb{I} \cong \mathbb{I}$ and the unit of the operad $u: \mathbb{I} \rightarrow \operatorname{Ass}^{\mathcal{V}}(1)$ is the identity. This operad is generated by the "elements" in degree 0 and 2 ; the degree 2 "element" represents the composition law, and the degree 0 "element" represents the identities. In order to simplify notation, we denote

$$
\operatorname{Cat}_{S}(\mathscr{V})=\operatorname{Alg}_{\operatorname{Graph}_{S}(\mathscr{V})}\left(\operatorname{Ass}^{\mathscr{V}}\right) .
$$

An $A_{\infty}$-category enriched on $\mathscr{V}$ with set of objects $S$ is an algebra over a cofibrant replacement $\mathrm{Ass}_{\infty}^{\mathcal{V}}$ of $\mathrm{Ass}^{\mathcal{V}}$, which is a trivial fibration $\phi: \mathrm{Ass}_{\infty}^{\mathcal{V}} \stackrel{\sim}{\rightarrow} \mathrm{Ass}^{\mathcal{V}}$ in $\mathrm{Op}(\mathscr{V})$ with cofibrant source. We simply denote

$$
A_{\infty}-\operatorname{Cat}_{S}(\mathscr{V})=\operatorname{Alg}_{\operatorname{Graph}_{S}(\mathscr{V})}\left(\operatorname{Ass}_{\infty}^{\mathcal{V}}\right)
$$

Combining the previous proposition with Theorem 1.2 we obtain the following corollary, which improves [6, Theorem 3.3].

Corollary 10.4 Let $\mathscr{V}$ be a cofibrantly generated closed symmetric monoidal category satisfying the monoid axiom. Suppose that $\mathscr{V}$ has sets of generating cofibrations and generating trivial cofibrations with presentable source. Then Cat ${ }_{S}(\mathscr{V})$ is a model category where an enriched functor $F: C \rightarrow D$ is a weak equivalence (resp. fibration) if $F(x, y): C(x, y) \rightarrow D(x, y)$ is a weak equivalence (resp. fibration) in $\mathscr{V}$ for all $x, y \in S$, and similarly for $A_{\infty}-\mathrm{Cat}_{S}(\mathcal{V})$. Moreover, these model categories are right proper (resp. combinatorial) provided $\mathscr{V}$ is.

The following corollary also uses Theorem 1.3.

Corollary 10.5 In the conditions of the previous corollary, assume in addition that $\mathscr{V}$ is left proper and the monoidal unit $\mathbb{I}_{\mathscr{V}}$ is cofibrant. Then the pullback functor $\phi^{*}$ from enriched categories to enriched $A_{\infty}$-categories and the strictification functor $\phi_{*}$ in the other direction form a Quillen equivalence

$$
A_{\infty}-\mathrm{Cat}_{S}(\mathscr{V}) \underset{\phi^{*}}{\stackrel{\phi_{*}}{\rightleftarrows}} \mathrm{Cat}_{S}(\mathscr{V})
$$


In particular, the derived adjoint pair is an equivalence between the homotopy categories of enriched categories and enriched $A_{\infty}$-categories:

$$
\text { Ho } A_{\infty}-\mathrm{Cat}_{S}(\mathscr{V}) \underset{\phi^{*}}{\stackrel{\mathbb{L} \phi_{*}}{\rightleftarrows}} \mathrm{HoCat}_{S}(\mathscr{V}) \text {. }
$$

When $\mathscr{C}$ is a simplicial model category and the simplicial structure is in a precise sense compatible with $z: \mathscr{V} \rightarrow Z(\mathscr{C})$, the derived equivalence of homotopy categories in this corollary was obtained in [2, Section 2] by different methods which are closer to categorical algebra than to homotopy theory.

\section{References}

[1] J Adámek, J Rosický, Locally presentable and accessible categories, London Math. Soc. Lecture Note Ser. 189, Cambridge Univ. Press (1994) MR1294136

[2] M A Batanin, Homotopy coherent category theory and $A_{\infty}$-structures in monoidal categories, J. Pure Appl. Algebra 123 (1998) 67-103 MR1492896

[3] H-J Baues, M Jibladze, A Tonks, Cohomology of monoids in monoidal categories, from: "Operads: Proceedings of Renaissance Conferences (Hartford, CT/Luminy, 1995)", (J-L Loday, J D Stasheff, A A Voronov, editors), Contemp. Math. 202, Amer. Math. Soc. (1997) 137-165 MR1436920

[4] C Berger, I Moerdijk, Axiomatic homotopy theory for operads, Comment. Math. Helv. 78 (2003) 805-831 MR2016697

[5] F Borceux, Handbook of categorical algebra. 2. Categories and structures, Encyclopedia of Math. and its Appl. 51, Cambridge Univ. Press (1994) MR1313497

[6] B I Dundas, Localization of V-categories, Theory Appl. Categ. 8 (2001) 284-312 MR1835445

[7] W G Dwyer, D M Kan, Calculating simplicial localizations, J. Pure Appl. Algebra 18 (1980) 17-35 MR578563

[8] W G Dwyer, D M Kan, Function complexes in homotopical algebra, Topology 19 (1980) 427-440 MR584566

[9] W G Dwyer, D M Kan, Simplicial localizations of categories, J. Pure Appl. Algebra 17 (1980) 267-284 MR579087

[10] V Ginzburg, M Kapranov, Koszul duality for operads, Duke Math. J. 76 (1994) 203-272 MR1301191

[11] J E Harper, Homotopy theory of modules over operads and non- $\Sigma$ operads in monoidal model categories, J. Pure Appl. Algebra 214 (2010) 1407-1434 MR2593672 
[12] V Hinich, Erratum to "Homological algebra of homotopy algebras" [13] arXiv: math/0309453v3

[13] V Hinich, Homological algebra of homotopy algebras, Comm. Algebra 25 (1997) 3291-3323 MR1465117

[14] P S Hirschhorn, Model categories and their localizations, Math. Surveys and Monogr. 99, Amer. Math. Soc. (2003) MR1944041

[15] M Hovey, Model categories, Math. Surveys and Monogr. 63, Amer. Math. Soc. (1999) MR1650134

[16] A Joyal, R Street, Tortile Yang-Baxter operators in tensor categories, J. Pure Appl. Algebra 71 (1991) 43-51 MR1107651

[17] G M Kelly, Basic concepts of enriched category theory, London Math. Soc. Lecture Note Ser. 64, Cambridge Univ. Press (1982) MR651714

[18] J-P Meyer, Bar and cobar constructions. I, J. Pure Appl. Algebra 33 (1984) 163-207 MR754954

[19] G Raptis, On the cofibrant generation of model categories, J. Homotopy Relat. Struct. 4 (2009) 245-253 MR2520994

[20] S Schwede, B E Shipley, Algebras and modules in monoidal model categories, Proc. London Math. Soc. (3) 80 (2000) 491-511 MR1734325

Facultad de Matemáticas, Departamento de Álgebra, Universidad de Sevilla Avda Reina Mercedes s/n, 41012 Sevilla, Spain

fmuro@us.es

http://personal.us.es/fmuro

Received: 14 January 2011 